Historic, Archive Document

Do not assume content reflects current scientific knowledge, policies, or practices. 

2SD II

\section{.1223}

USDA United States Department of Agriculture

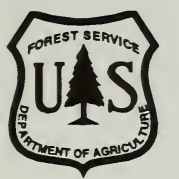

Forest Service Alaska Region

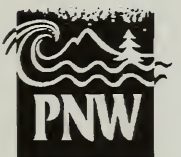

Pacific

Northwest

Research

Station

Vascular Plant

Identification

Guide

Chugach National

Forest

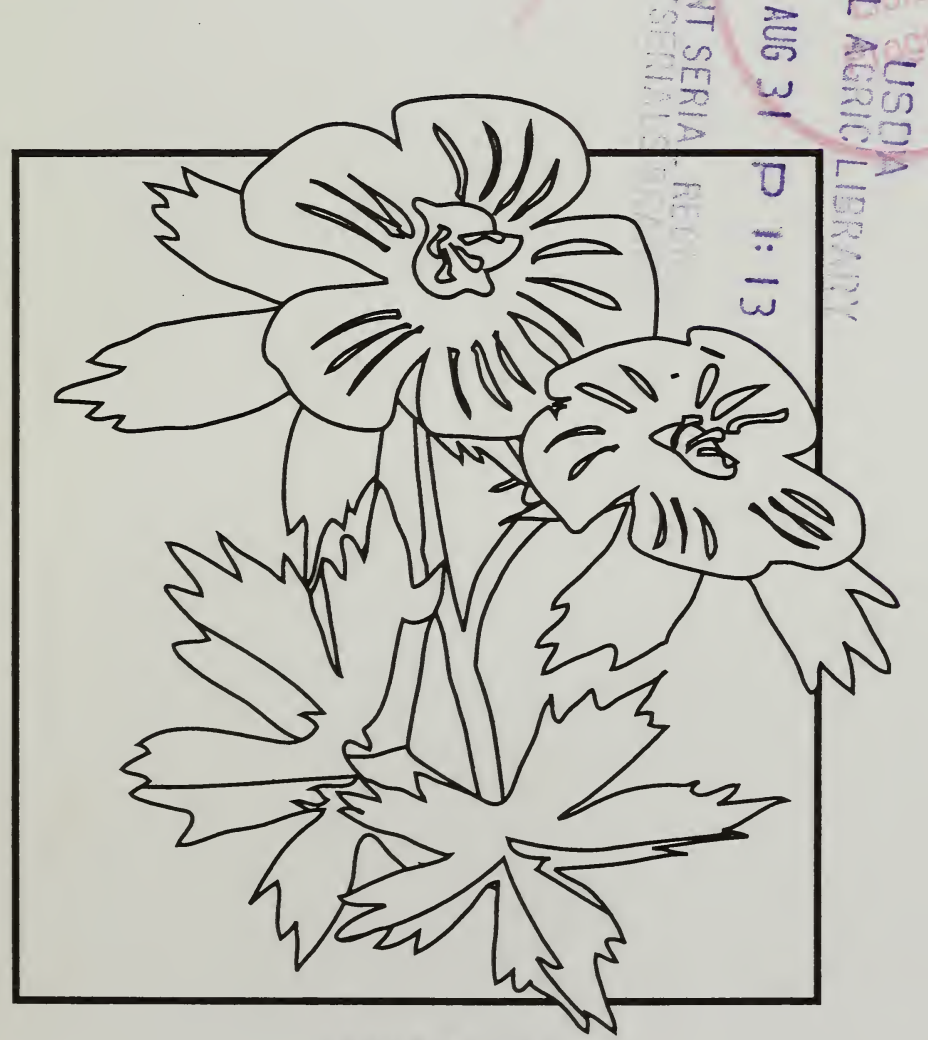

R10-MB-421 


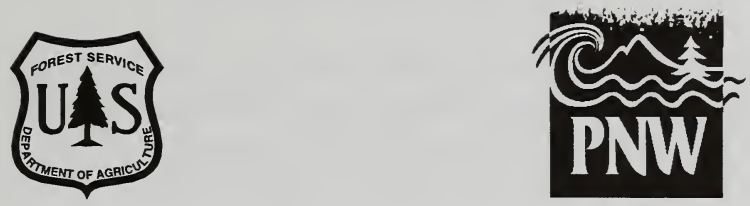

\title{
VASCULAR PLANT IDENTIFICATION GUIDE CHUGACH NATIONAL FOREST
}

\author{
Robert L. DeVelice ${ }^{1}$ \\ Susan L. Boudreau² \\ Charles Wertheim ${ }^{3}$ \\ Connie J. Hubbard ${ }^{4}$ \\ Chrystal Czarnecki 5
}

\author{
May 2001 \\ USDA Forest Service \\ Chugach National Forest \\ 3301 C Street, Suite 300 \\ Anchorage, Alaska 99503-3998
}

1USDA Forest Service, Chugach National Forest

${ }^{2}$ USDI National Park Service, Denali National Park

3private consultant, Glenwood Springs, Colorado

${ }^{4}$ USDA Forest Service, Pacific Northwest Research Station

${ }^{5}$ Rutgers University, New Brunswick, New Jersey 
The U.S. Department of Agriculture (USDA) prohibits discrimination in all its programs and activities on the basis of race, color, national origin, sex, religion, age, disability, political beliefs, sexual orientation, or marital or family status. (Not all prohibited bases apply to all programs.) Persons with disabilities who require alternative means for communication of program information (Braille, large print, audiotape, etc.) should contact USDA's TARGET Center at (202) 720-2600 (voice and TDD).

To file a complaint of discrimination, write USDA, Director, Office of Civil Rights, Room 326-W, Whitten Building, 1400 Independence Avenue, SW, Washington, D.C. 20250-9410 or call (202) 720-5964 (voice and TDD). USDA is an equal opportunity provider and employer. 


\section{ACKNOWLEDGEMENTS}

We acknowledge John DeLapp, Tom DeMeo, Jon Martin, Bill Pawuk, Beth Schulz, and Mary Stensvold for their valuable suggestions and helpful reviews. We extend special appreciation to field crews, without whose hard work the data upon which this guide is based would not have existed. We appreciate the assistance Mona Spargo provided in the layout and final preparation of this document. We also thank the USDA Forest Service Alaska Region, Chugach National Forest, and the Forestry Sciences Laboratory of the Pacific Northwest Research Station for their encouragement and support.

The Forestry Sciences Laboratory, Pacific Northwest Research Station, Anchorage, Alaska generously provided funding for printing this guide.

All line drawings are from Flora of Alaska and Neighboring Territories: A Manual of the Vascular Plants, by Eric Hultén, and are used with the permission of the publishers, Stanford University Press (C) 1968 by the Board of Trustees of the Leland Stanford Junior University. 



\section{TABLE OF CONTENTS}

\section{INTRODUCTION}

\section{TREES}

Betula kenaica

Betula papyrifera

Chamaecyparis nootkatensis

Picea glauca

Picea X lutzii

Picea mariana

Picea sitchensis

Populus balsamifera subsp. balsamifera

Populus balsamifera subsp. trichocarpa

Populus tremuloides

Salix scouleriana

Tsuga heterophylla

Tsuga mertensiana

\section{SHRUBS}

Alnus crispa subsp. sinuata

Alnus incana subsp. tenuifolia

Amelanchier spp.

Andromeda polifolia

Arctostaphylos alpina

Arctostaphylos rubra

Arctostaphylos uva-ursi

Betula glandulosa/nana

Cassiope mertensiana

Cassiope stelleriana

Cassiope tetragona

Cladothamnus pyrolaeflorus

Diapensia lapponica

Dryas octopetala

Echinopanax horridum

Empetrum nigrum

Juniperus communis

Ledum palustre subsp. decumbens

Ledum palustre subsp. groenlandicum

Linnaea borealis

Loiseleuria procumbens
Kenai birch 4

paper birch 4

Alaska yellow cedar 5

white spruce 5

Lutz spruce 6

black spruce $\quad 6$

Sitka spruce 7

balsam poplar $\quad 7$

black cottonwood 8

quaking aspen $\quad 8$

Scouler willow 9

western hemlock 9

mountain hemlock 10

Sitka alder $\quad 13$

thinleaf alder $\quad 13$

serviceberry 14

bog rosemary 14

alpine bearberry 15

red bearberry $\quad 15$

kinnikinnick 16

dwarf birches $\quad 16$

Mertens cassiope $\quad 17$

Steller's cassiope $\quad 17$

four-angled cassiope $\quad 18$

copperbush 18

diapensia 19

white mountain-avens 19

devil's club 20

crowberry 20

common juniper 21

narrow-leaf Labrador-tea 21

Greenland Labrador-tea 22

twinflower 22

alpine azalea 23

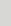

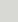

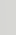

7

8

(1)

(1)


Luetkea pectinata

Malus fusca

Menziesia ferruginea

Myrica gale

Oxycoccus microcarpus

Phyllodoce aleutica

Potentilla fruticosa

Ribes bracteosum

Ribes glandulosum

Ribes hudsonianum

Ribes lacustre

Ribes laxiflorum

Ribes triste

Rosa acicularis

Rubus idaeus

Rubus spectabilis

Salix alaxensis

Salix arctica

Salix barclayi

Salix commutata

Salix depressa subsp. rostrata

Salix hookeriana

Salix myrtillifolia

Salix pulchra

Salix reticulata

Salix rotundifolia

Salix sitchensis

Sambucus racemosa

Shepherdia canadensis

Sibbaldia procumbens

Sorbus scopulina

Sorbus sitchensis

Spiraea beauverdiana

Vaccinium alaskensis

Vaccinium caespitosum

Vaccinium ovalifolium

Vaccinium uliginosum

Vaccinium vitis-idaea

Viburnum edule luetkea

Oregon crab apple

rusty menziesia

sweetgale

bog cranberry

Aleutian mountain heath

shrubby cinquefoil

stink currant

skunk currant

northern black currant

bristly black currant

trailing black currant

northern red currant

prickly rose

raspberry

salmonberry

feltleaf willow

arctic willow

Barclay willow

undergreen willow

Bebb willow

Hooker willow

low blueberry willow

tealeaf willow

netleaf willow

least willow

Sitka willow

elderberry

buffaloberry

creeping sibbaldia

western mountain-ash 38

Sitka mountain-ash $\quad 39$

Beauverd spiraea $\quad 39$

Alaska blueberry $\quad 40$

dwarf blueberry $\quad 40$

early blueberry 41

bog blueberry 41

lowbush cranberry $\quad 42$

highbush cranberry $\quad 42$ 
Achillea borealis

Aconitum delphinifolium

Actaea rubra

Anemone narcissiflora

Anemone richardsonii

Angelica genuflexa

Angelica lucida

Antennaria monocephala

Apargidium boreale

Aquilegia formosa

Arabis lyrata

Arnica latifolia

Artemisia arctica

Artemisia tilesii

Aruncus sylvester

Boschniakia rossica

Callitriche hermaphroditica

Callitriche verna

Caltha palustris

Campanula lasiocarpa

Campanula rotundifolia

Cardamine umbellata

Castilleja unalaschcensis

Cicuta douglasii

Cicuta mackenzieana

Circaea alpina

Coeloglossum viride

Conioselinum chinense

Coptis aspleniifolia

Coptis trifolia

Cornus canadensis

Delphinium glaucum

Dodecatheon pulchellum

Drosera rotundifolia

Epilobium adenocaulon

Epilobium anagalladifolium

Epilobium angustifolium

Epilobium glandulosum

Epilobium hornemannii

Epilobium latifolium

Epilobium leptocarpum yarrow

monkshood 45

baneberry 46

narcissus anemone $\quad 46$

yellow anemone $\quad 47$

bent-leaved angelica $\quad 47$

seacoast angelica $\quad 48$

pygmy pussytoes $\quad 48$

apargidium 49

western columbine $\quad 49$

lyreleaved rockcress $\quad 50$

broadleaf arnica $\quad 50$

boreal sagebrush $\quad 51$

Aleutian mugwort 51

goatsbeard 52

ground-cone 52

northern waterstarwort 53

spring waterstarwort 53

yellow marsh-marigold $\quad 54$

mountain harebell $\quad 54$

bluebells of Scotland 55

little western bittercress $\quad 55$

Alaska Indian paintbrush $\quad 56$

western water hemlock 56

Mackenzie's water hemlock $\quad 57$

enchanter's nightshade $\quad 57$

longbract frog orchid $\quad 58$

western hemlock-parsley $\quad 59$

fern leaf goldenthread $\quad 59$

trifoliate goldenthread $\quad 60$

bunchberry $\quad 60$

larkspur $\quad 61$

pretty shooting star $\quad 61$

round-leaf sundew 61

northern willow herb $\quad 62$

alpine willow herb $\quad 62$

tall fireweed 63

fringed willow herb 63

Hornemann's willow herb $\quad 64$

dwarf fireweed 64

slenderfruit willow herb $\quad 65$ 
Epilobium palustre

Erigeron peregrinus

Fauria crista-galli

Fragaria chiloensis

Fritillaria camschatcensis

Galium boreale

Galium trifidum

Galium triflorum

Gentiana douglasiana

Gentiana glauca

Gentiana platypetala

Geocaulon lividum

Geranium erianthum

Geum calthifolium

Geum macrophyllum

Hedysarum alpinum

Heracleum lanatum

Heuchera glabra

Hieracium triste

Hippuris tetraphylla

Hippuris vulgaris

Honckenya peploides

Iris setosa

Lathyrus maritimus

Lathyrus palustris

Ligusticum scoticum

Listera borealis

Listera caurina

Listera cordata

Lloydia serotina

Lupinus nootkatensis

Lysichiton americanum

Lysimachia thyrsiflora

Maianthemum dilatatum

Menyanthes trifoliata

Mertensia paniculata

Minuartia arctica

Moehringia lateriflora

Moneses uniflora

Myosotis alpestris

Myriophyllum sibiricum

Nuphar polysepala

Osmorhiza depauperata

swamp willow herb

subalpine fleabane

deer cabbage

beach strawberry

chocolate lily

northern bedstraw $\quad 68$

threepetal bedstraw $\quad 68$

fragrant bedstraw $\quad 69$

swamp gentian $\quad 69$

pale gentian $\quad 70$

spotted gentian $\quad 70$

northern comandra $\quad 71$

northern geranium 71

calthaleaf avens $\quad 72$

large-leaf avens $\quad 72$

alpine sweetvetch $\quad 73$

cow parsnip $\quad 73$

alpine heuchera $\quad 74$

woolly hawkweed $\quad 74$

fourleaf marestail $\quad 75$

common marestail $\quad 75$

seaside sandplant $\quad 76$

wild iris $\quad 76$

beach pea $\quad 77$

vetchling $\quad 77$

beach lovage $\quad 78$

northern twayblade $\quad 78$

western twayblade $\quad 79$

heart-leaf twayblade $\quad 79$

common alplily $\quad 80$

Nootka lupine $\quad 80$

skunk cabbage $\quad 81$

tufted loosestrife $\quad 81$

deerberry $\quad 82$

buckbean $\quad 82$

chiming bells $\quad 83$

arctic stitchwort $\quad 83$

grove sandwort $\quad 84$

single delight $\quad 84$

forget-me-not 85

shortspike watermilfoil $\quad 85$

yellow pondlily $\quad 86$

blunt-fruit sweet-cicely $\quad 86$ 
Osmorhiza purpurea

Oxytropis nigrescens

Parnassia palustris

Pedicularis capitata

Pedicularis kanei

Pedicularis labradorica

Pedicularis langsdorfii

Pedicularis parviflora

Pedicularis verticillata

Petasites hyperboreus

Pinguicula vulgaris

Plantago maritima

Platanthera dilitata

Platanthera saccata

Polemonium acutiflorum

Polygonum viviparum

Potamogeton filiformis

Potamogeton natans

Potamogeton pectinatus

Potamogeton perfoliatus

Potentilla egedii

Potentilla palustris

Prenanthes alata

Primula cuneifolia

Pyrola asarifolia

Pyrola chlorantha

Pyrola grandiflora

Pyrola minor

Pyrola secunda

Ranunculus cymbalaria

Ranunculus occidentalis

Ranunculus trichophyllus

Rhinanthus minor

Rubus arcticus

Rubus chamaemorus

Rubus pedatus

Rumex fenestratus

Sanguisorba stipulata

Saxifraga bronchialis

Saxifraga punctata

Saxifraga tricuspidata

Sedum rosea

Senecio triangularis
Sitka sweet-cicely

blackish oxytrope

northern grass-of-parnassas $\quad 88$

capitate lousewort

woolly lousewort

Labrador lousewort $\quad 89$

Langsdorf's lousewort 90

smallflower lousewort $\quad 90$

whorled lousewort $\quad 91$

arctic sweet coltsfoot $\quad 91$

common butterwort 92

goosetongue plantain 92

boreal bog orchid 93

slender bog orchid 93

tall Jacob's-ladder 94

alpine bistort 94

slender-leaved pondweed 95

floating pondweed 95

sago pondweed 96

claspingleaf pondweed 96

Pacific silverweed 97

marsh fivefinger 97

rattlesnake root 98

wedgeleaf primrose $\quad 98$

liverleaf wintergreen $\quad 99$

greenish wintergreen $\quad 99$

largeflowered wintergreen $\quad 100$

snowline wintergreen $\quad 100$

one-sided wintergreen $\quad 101$

seaside buttercup $\quad 101$

western buttercup 102

white water crowfoot $\quad 102$

yellow rattle 103

nagoonberry 103

cloudberry 104

fiveleaf bramble $\quad 104$

western dock 105

Sitka burnet 105

yellowdot saxifrage $\quad 106$

heart-leaved saxifrage $\quad 106$

prickly saxifrage $\quad 107$

roseroot stonecrop $\quad 107$

arrowleaf groundsel $\quad 108$ 
Solidago multiradiata

Sparganium angustifolium

Sparganium hyperboreum

Sparganium minimum

Spiranthes romanzoffiana

Stellaria calycantha

Stellaria crassifolia

Stellaria crispa

Stellaria sitchana

Streptopus amplexifolius

Subularia aquatica

Swertia perennis

Thalictrum sparsiflorum

Tiarella trifoliata

Tofieldia coccinea

Tofieldia glutinosa

Trientalis europaea

Triglochin maritimum

Utricularia vulgaris

Valeriana sitchensis

Veratrum viride

Viola epipsila

Viola glabella

Viola langsdorffii

\section{GRAMINOIDS}

Agrostis aequivalvis

Agrostis alaskana

Arctagrostis latifolia

Arctophila fulva

Calamagrostis canadensis

Calamagrostis nutkaënsis

Carex anthoxanthea

Carex aquatilis

Carex lyngbyaei

Carex macrochaeta

Carex magellanica

Carex microchaeta

Carex microglochin

Carex pauciflora

Carex pluriflora

Carex sitchensis

Deschampsia caespitosa northern goldenrod

floating burreed

northern burreed

small burreed

hooded ladies' tresses

northern starwort

fleshy starwort

curled starwort

Sitka starwort

twistedstalk

awlwort

star gentian

fewflower meadowrue

foamflower

northern asphodel

sticky false-asphodel

starflower

seaside arrowgrass

common bladderwort

Sitka valerian

false hellebore

marsh violet

yellow violet

Alaska violet

arctic bentgrass

Alaska bentgrass

polargrass

pendant grass

bluejoint reedgrass $\quad 126$

Pacific reedgrass

arctic sedge

water sedge

Lyngbye's sedge

longawned sedge

boreal bog sedge

smallawned sedge

fewseeded bog sedge

fewflower sedge

manyflower sedge

Sitka sedge

tufted hairgrass 
Eleocharis acicularis

Eleocharis palustris

Elymus arenarius

Eriophorum angustifolium

Eriophorum russeolum

Festuca altaica

Festuca rubra

Glyceria pauciflora

Hierochloë alpina

Hordeum brachyantherum

Juncus alpinus

Juncus arcticus

Luzula multiflora

Luzula parviflora

Luzula wahlenbergii

Phleum commutatum

Poa lanata

Poa macrantha

Puccinellia nutkaensis

Puccinellia pumila

Trichophorum caespitosum

Trisetum spicatum

Vahlodea atropurpurea

FERNS AND FERN ALLIES

Athyrium filix-femina

Blechnum spicant

Cystopteris fragilis

Dryopteris dilatata

Equisetum arvense

Equisetum fluviatile

Equisetum palustre

Equisetum pratense

Equisetum sylvaticum

Equisetum variegatum

Gymnocarpium dryopteris

Lycopodium alpinum

Lycopodium annotinum

Lycopodium clavatum

Lycopodium complanatum

Lycopodium sabinifolium

Lycopodium selago

Selaginella selaginoides needle spikerush

common spikerush

133

beach rye

133

tall cottongrass

134

red cottongrass

134

rough fescue

135

red fescue

135

false mannagrass

136

alpine holygrass

136

meadow barley

137

alpine rush

137

arctic rush

138

common woodrush

138

smallflowered woodrush

139

Wahlenberg's woodrush

139

mountain timothy

140

arctic bluegrass

140

seashore bluegrass

141

Nootka alkaligrass

dwarf alkaligrass

142

tufted bulrush

142

spike trisetum

143

mountain hairgrass

143

145

lady fern

deer fern

146

fragile fern

147

wood fern

147

common horsetail

148

swamp horsetail

148

marsh horsetail

149

meadow horsetail

149

woodland horsetail

150

northern horsetail

150

oak fern

151

alpine clubmoss

stiff clubmoss

152

running clubmoss

152

groundcedar

153

savinleaf groundpine $\quad 153$

fir clubmoss

club spikemoss 


\section{APPENDICES}

Appendix 1. Comparison between Hultén and PLANTS nomenclature Appendix 4. Comparison among stink, trailing black, northern black, and northern red currant

Appendix 5. Comparison between Alaska and early blueberry Appendix 6. Glossary 


\section{INTRODUCTION}

This book is primarily a visual and descriptive guide to vascular plant species commonly found on the Chugach National Forest. This book is intended for all users, not just botanists, and as such, technical terminology has been avoided whenever possible. For more comprehensive descriptions and keys to the plants we suggest Flora of Alaska and Neighboring Territories: A Manual of the Vascular Plants (Hultén, 1968).

An earlier version of this guide was entitled Forest Plant Identification Guide: Chugach National Forest (Borchers and Wertheim 1992) and featured 194 taxa. We have revised the earlier guide by expanding the scope to include plants predominately found in non-forest settings and other forest species found to be common on the National Forest. A total of 284 taxa are included.

Included are woody species occurring on five or more of 2300 sample sites; forbs, ferns, and fern allies occurring on 10 or more of the sites; and graminoids (grass and grass-like plants) occurring on 20 or more of the sites. Also included are less common species if they are used in the names or descriptions of community types in DeVelice et al. (1999).

This guide is organized by life forms: trees; shrubs; forbs; graminoids; and ferns and fern allies. Names are organized alphabetically by genus and species within each life form. Descriptions are presented in the following format:

GENUS AND SPECIES

COMMON NAME

\section{SPECIES CODE}

FAMILY

HABIT: Size range and general appearance of the species.

KEY CHARACTERS: Description of the species.

HABITAT: Description of the most frequently occupied habitat.

Scientific nomenclature follows Hultén (1968). Common names are from various sources. Species codes are as provided in the PLANTS database (USDA, NRCS 1999). For 58 species, the PLANTS database provides revised scientific nomenclature from that in Hultén. A crosswalk table listing these revisions (based on a February 5, 2001 download from the PLANTS database) is in the Appendices.

Also included are summary tables, located in the Appendices, of major characteristics of genera that contain a number of species that are often confused.

A Glossary is located in the Appendices that will help the user become acquainted with terms. 
TREES 
Betula kenaica

Kenai birch
BEKE2

Betulaceae

HABIT: Deciduous, 20-80 feet.

KEY CHARACTERS: Leaves alternate, doubly toothed, short-pointed with hairs above. Twigs with reddish hairs and resin dots when young. Inflorescence a short, narrow, dark brown catkin. Fruit cone-like, 1 inch long, upright or spreading. Bark dark brown and papery.

HABITAT: Subalpine, in alder belt.

Betula papyrifera paper birch
BEPA

Betulaceae

HABIT: Deciduous, 20-80 feet.

KEY CHARACTERS: Leaves alternate, toothed, long-pointed, hairless above. Twigs with many resin dots. Inflorescence a short, thick, greenish-brown catkin. Fruit conelike, hairless, 1-13/3 inches long, hanging down or spreading. Bark white and papery.

HABITAT: Low elevation to treeline. Prefers warm slopes with moist porous soils, also common on cold north slopes and poorly drained lowlands. Common pioneer after burns. In pure stands or with white or black spruce.

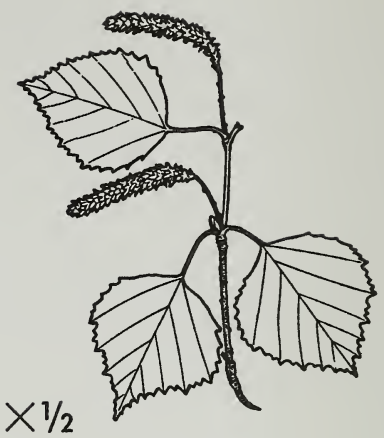


Chamaecyparis nootkatensis CHNO

Alaska yellow cedar

Cupressaceae

HABIT: Evergreen, 40-80 feet.

KEY CHARACTERS: Leaves yellowgreen, scale-like, pointed, and spreading. Leafy twigs four-angled. Branches droop. Cones small, round, with 4-6 scales. Wood with "potato-like" odor.

HABITAT: Scattered in small groups in coastal forest from sea level to treeline but best developed below 1200 feet. In pure stands or with birch.

Picea glauca white spruce

PIGL

Pinaceae

HABIT: Evergreen, 35-85 feet.

KEY CHARACTERS: Needles $1 / 2-3 / 4$ inches long, four-angled, pointed, with white lines on all sides. Twigs hairless, with needles massing on top near the ends. Needles and twigs with skunk-like odor. Cones $1 \frac{1}{4}-2 \frac{1}{2}$ inches long, deciduous, cylindrical, nearly stalkless, with thin, round, smooth, flexible scales. Inner bark white.

HABITAT: Sea level to treeline. Prefers river bottoms, sandy lakeshores, and welldrained gentle south slopes.
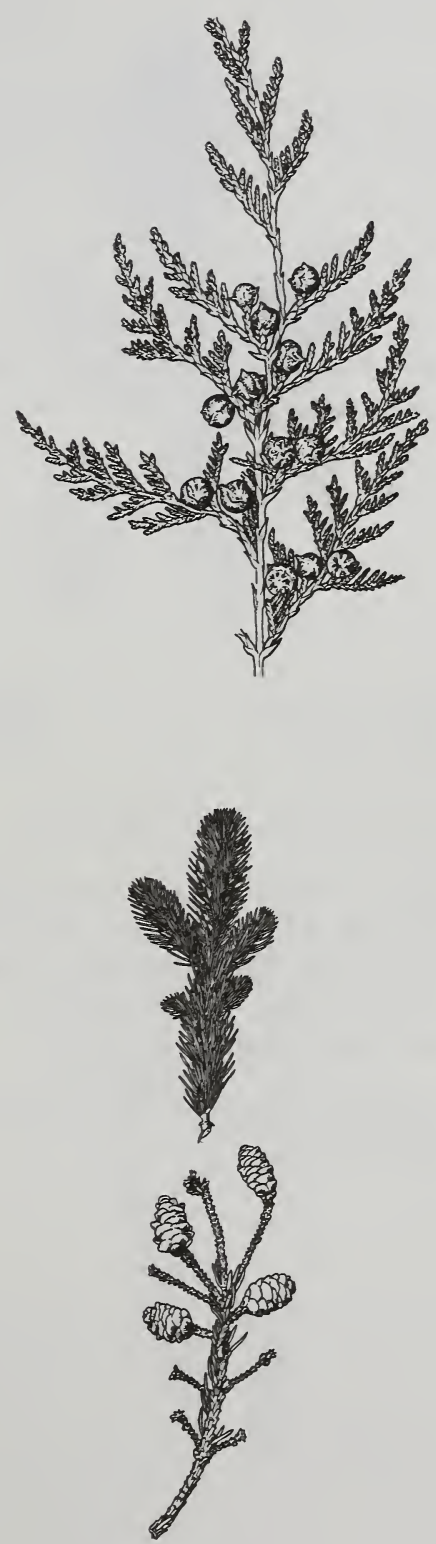
Picea X lutzii

Lutz spruce
PILU

Pinaceae

A natural hybrid between white spruce and Sitka spruce, with most characteristics intermediate between the two species.

HABIT: Evergreen, 55-100 feet.

KEY CHARACTERS: Needles slightly four-angled with white upper surface. Cones scales short and toothed.

Picea mariana black spruce
PIMA

Pinaceae

HABIT: Evergreen, 15-30 feet.

KEY CHARACTERS: Needles short, squarish in cross-section, with white lines on all sides. Twigs red hairy. Cones 1 inch long, rounded, dark, remaining on tree several years. Inner bark yellow.

HABITAT: Muskegs, wet meadows, lakeshores.

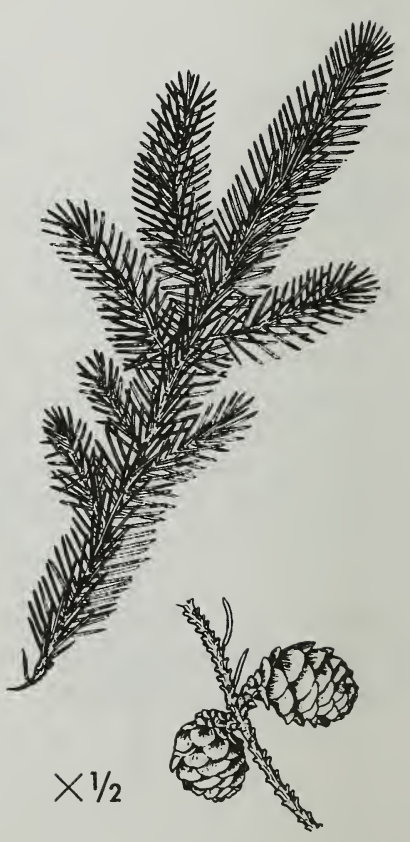


Picea sitchensis

Sitka spruce
PISI

Pinaceae

HABIT: Evergreen, 80-150 feet.

KEY CHARACTERS: Needles $3 / 4-1$ inch long, flattened, keeled underneath, sharp pointed, white above. Twigs hairless. Cones $2-3 \frac{1}{2}$ inches long, cylindric-oblong, with long, thin, stiff, rounded, and toothed scales. Inner bark white with brown dots.

HABITAT: Chiefly below 1500 feet, but up to 3000 feet. In pure stands or with hemlock or cottonwood.

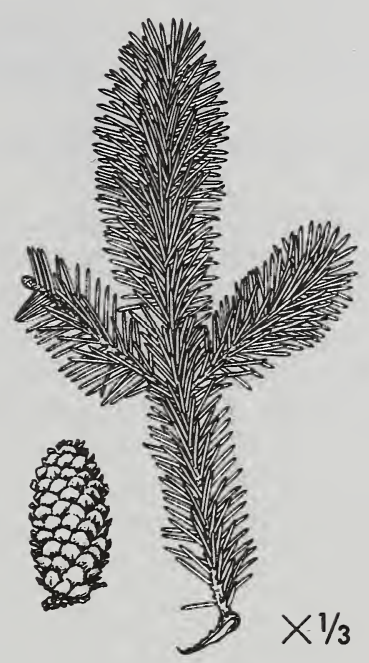

Populus balsamifera subsp. balsamifera balsam poplar

POBAB2

Salicaceae

HABIT: Deciduous, $30-45$ feet.

KEY CHARACTERS: Leaves alternate, pale and rusty brown underneath, with small rounded teeth. Winter buds 1 inch long, sticky, pointed, odorous. Inflorescence a $2-3 \frac{1}{2}$ inch long, drooping catkin. Fruit hairless 2-parted capsules on 6 inch long catkins. Bark thick, grey, deeply furrowed.

HABITAT: River valleys, lakeshores, alluvial flats.

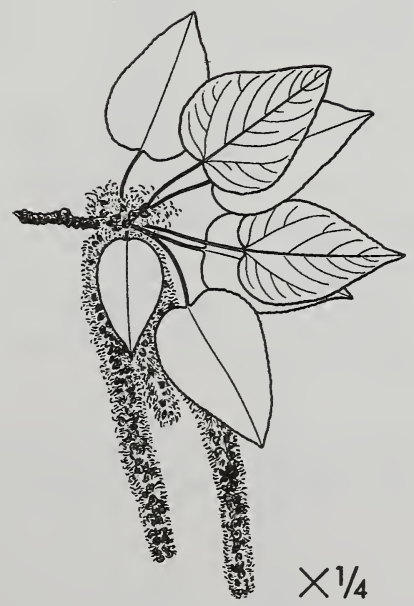


Populus balsamifera subsp. trichocarpa black cottonwood

POBAT

Salicaceae

HABIT: Deciduous, 80-100 feet.

KEY CHARACTERS: Leaves alternate, pale and rusty brown underneath, with small rounded teeth. Winter buds $3 / 4$ inches long, sticky, pointed. Inflorescence a $1 \frac{1}{2}-3$ inch long drooping catkin. Fruit 3-parted capsules with white hairs, on 6 inch long catkins. Bark thick, grey, deeply furrowed.

HABITAT: Open forests, river bottoms.

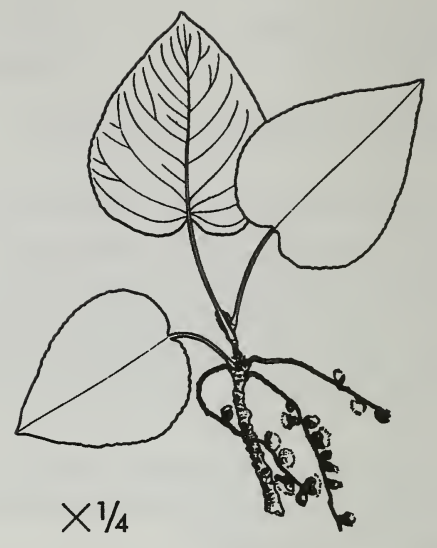

Populus tremuloides

POTR5

quaking aspen

Salicaceae

HABIT: Deciduous, 20-40 feet.

KEY CHARACTERS: Leaves with flat petiole and small rounded teeth.

Inflorescence $1-2 \frac{1}{2}$ inch long drooping catkin. Fruit 2-parted capsules on 4 inch long catkins. Bark smooth, pale, yellowishgreen.

HABITAT: Open forests, streambanks, south slopes. Common pioneer after burns.

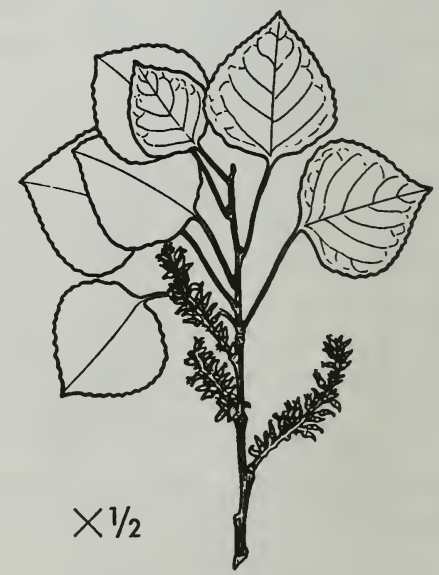


Salix scouleriana

Scouler willow
SASC

Salicaceae

HABIT: Deciduous, 30-60 feet.

KEY CHARACTERS: Leaves alternate, red pubescent underneath, crowded at ends of twigs. Buds red, without bud scales. Fruit woolly capsules on 2 inch long, stalkless catkins. Bark grey and smooth.

HABITAT: Forests, muskegs, burned areas.

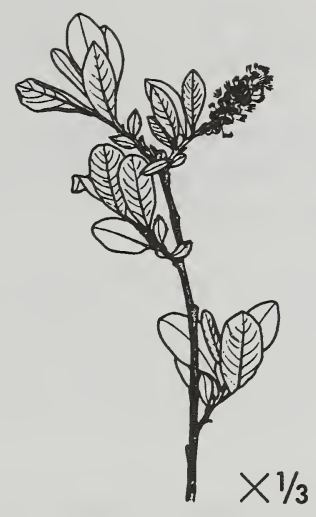

Tsuga heterophylla western hemlock

\section{TSHE}

Pinaceae

HABIT: Evergreen, 100-120 feet.

KEY CHARACTERS: Needles in 2 rows on twigs, flattened, white underneath. Leading branch at top of tree droops. Cones $3 / 4-1$ inch long. Inner bark red. Bark reddish-brown.

HABITAT: Sea level to subalpine, below mountain hemlock zone. Prefers moist flats and low slopes. In stands with Sitka spruce and mountain hemlock.

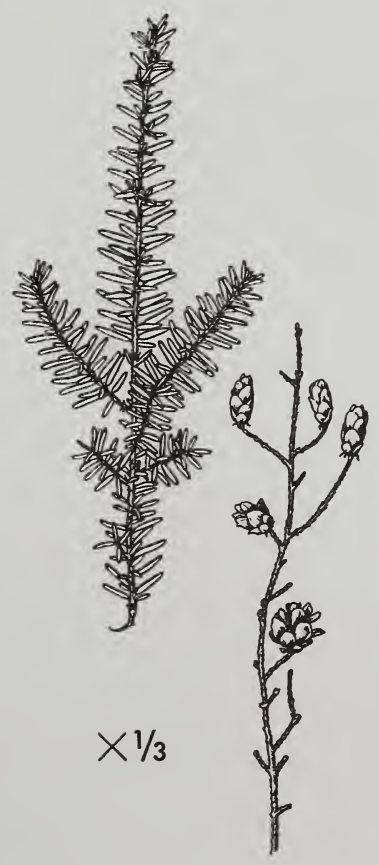


Tsuga mertensiana mountain hemlock
TSME

Pinaceae

HABIT: Evergreen, 50-100 feet.

Sometimes 10 feet tall or less in musegs and at upper treeline.

KEY CHARACTERS: Needles on all sides of twigs, keeled, curved, white on both sides. Leading branch at top of tree droops. Cones 1-3 inches long. Bark gray to dark-

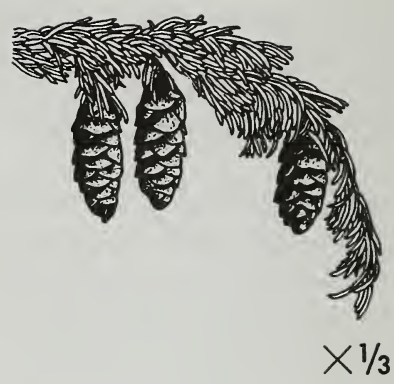
brown.

HABITAT: Sea level to 3500 feet, extending higher than western hemlock. Muskegs and mountain slopes. 


\section{SHRUBS}


Alnus crispa subsp. sinuata Sitka alder

\section{ALCRS}

Betulaceae

HABIT: Deciduous, ascending, manystemmed shrub, 5-25 feet.

KEY CHARACTERS: Leaves alternate, doubly toothed, shiny, sticky when young, with pointy lobes. Stems usually curved. Inflorescence a 3-5 inch catkin. Fruit cone-

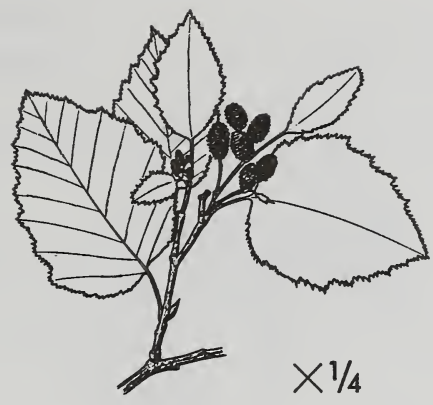
like on stalks longer than $1 / 4$ inch.

HABITAT: Sea level to alpine.

Streambanks, disturbed areas, shorelines, slopes, avalanche shoots.

Alnus incana subsp. tenuifolia thinleaf alder
ALINT

Betulaceae

HABIT: Deciduous large shrub or small tree, 15-30 feet.

KEY CHARACTERS: Leaves alternate, doubly toothed, with rounded lobes, often with reddish color. Inflorescence a narrow, $1 \frac{1}{2}-3$ inch long catkin. Fruit cone-like on stalks shorter than $1 / 4$ inch.

HABITAT: Open forests, streambanks.

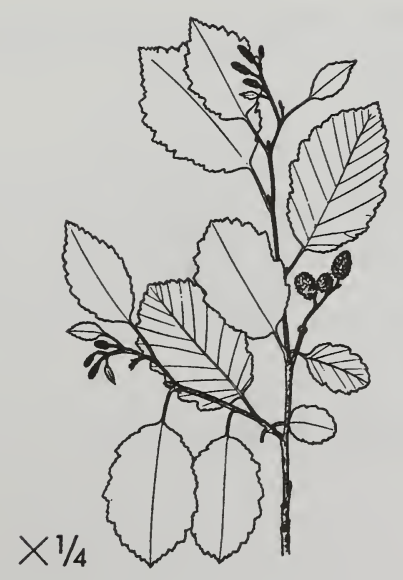


Amelanchier spp. serviceberry
AMELA

Rosaceae

HABIT: Deciduous low shrub to small tree, 1-15 feet.

KEY CHARACTERS: Leaves alternate, nearly round, with teeth toward tip. Flowers white, fragrant. Fruits apple-like, round, purplish-black, less than $3 / 4$ inch in diameter.

HABITAT: Open forests, meadows.

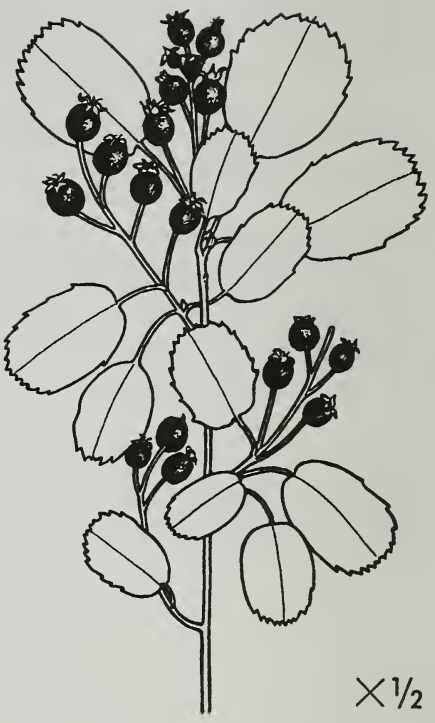

Andromeda polifolia bog rosemary
ANPO

Ericaceae

HABIT: Evergreen, creeping shrub with ascending branches, Up to 1 foot (usually shorter).

KEY CHARACTERS: Leaves alternate, whitish underneath, edges rolled under, thick, with sunken veins above. Flowers pink, in terminal inflorescences, nodding. Fruit $1 / 4$ inch diameter, round, 5-parted capsules.

HABITAT: Muskegs.

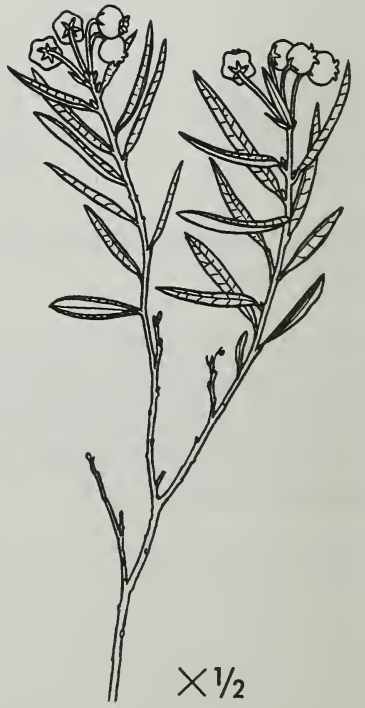


Arctostaphylos alpina

alpine bearberry
ARAL2

Ericaceae

HABIT: Deciduous prostrate shrub, 2-4 inches.

KEY CHARACTERS: Leaves alternate, whitish underneath, toothed, thick, distinctly veined, with hairy edges. Flowers white, few, terminal, nodding. Fruit round, black berries.

HABITAT: Alpine tundra.

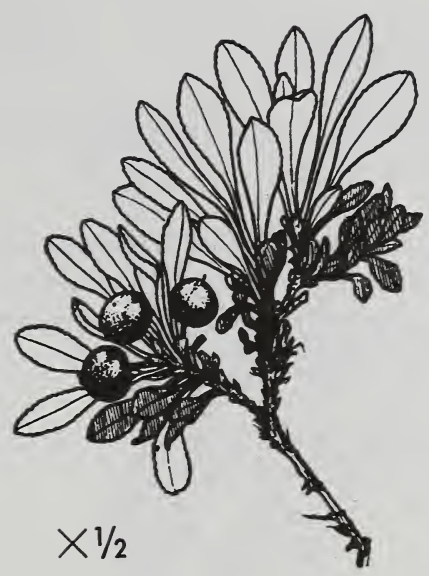

Arctostaphylos rubra red bearberry

ARRU

Ericaceae

HABIT: Deciduous prostrate shrub, to 6 inches.

KEY CHARACTERS: Leaves alternate, whitish underneath, toothed, thin, distinctly veined, without hairy edges. Flowers white, few, terminal, nodding. Fruit round, red berries.

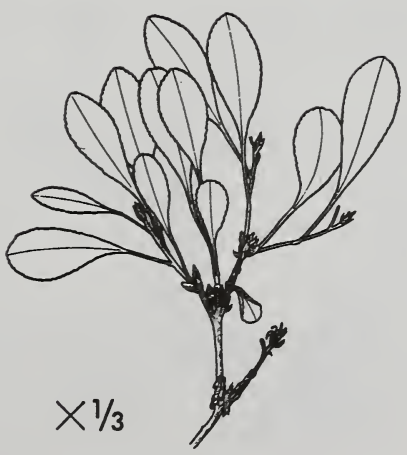

HABITAT: Spruce forests, muskegs. 


\section{Arctostaphylos uva-ursi \\ ARUV \\ kinnikinnick \\ Ericaceae}

HABIT: Evergreen prostrate shrub, 3-4 inches.

KEY CHARACTERS: Leaves alternate, spatula-shaped, leathery, distinctly veined. Flowers white to pink, in few-flowered terminal inflorescences. Fruit dry, red berries.

HABITAT: Open spruce forests, dry sandy places with aspen.

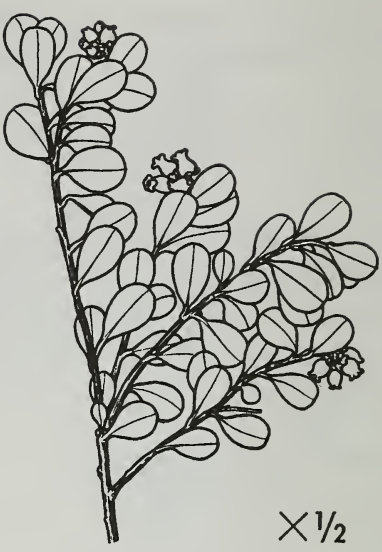

Betula glandulosa/nana

BETUL dwarf birches

Betulaceae

Betula glandulosa and $B$. nana hybridize extensively with each other, making identification difficult. The dwarf birches also hybridize with the tree birches.

HABIT: Deciduous low spreading to upright shrub, 1-5 feet.

KEY CHARACTERS: Leaves alternate, less than 1 inch long, thick, hairless, with rounded teeth. Twigs covered with resin dots. Inflorescence a catkin. Fruits conelike.

HABITAT: Muskegs, swamps, wet areas, rocky alpine slopes, tundra.

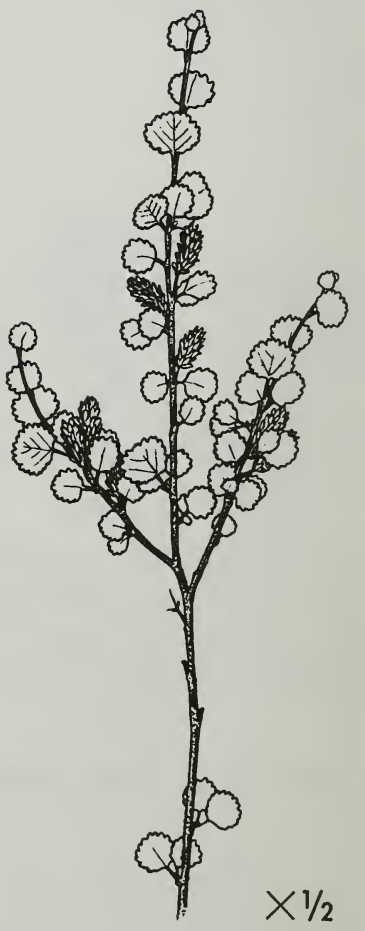


Cassiope mertensiana

Mertens cassiope

HABIT: Evergreen mat-forming shrub, 2-12 inches.

KEY CHARACTERS: Leaves opposite, in 4 rows, $1 / 8$ inch long, scale-like, completely covering stem. Flowers white to pink, several per stem, solitary, from leaf bases.

Fruit $1 / 8$ inch diameter, round, upright capsules.

HABITAT: To 3900 feet. Mountain meadows, slopes adjacent to snowfields.
CAME7

Ericaceae

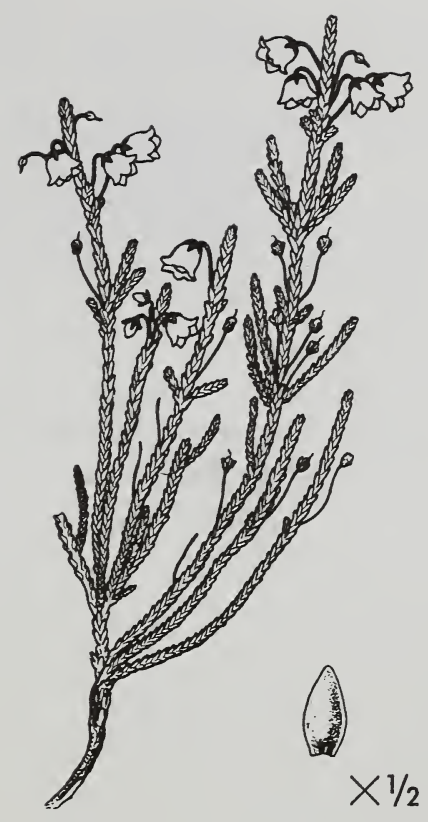

Cassiope stelleriana Steller's cassiope

CAST33

Ericaceae

HABIT: Evergreen, mat-forming shrub, $2-4$ inches.

KEY CHARACTERS: Leaves alternate, spreading, $1 / 4$ inch long, needle-like. Flowers white to pinkish, 1 per stem, terminal. Fruit $1 / 8$ inch diameter, round, upright capsules.

HABITAT: To 6000 feet. Forests near treeline, heaths, snow depressions, protected slopes.

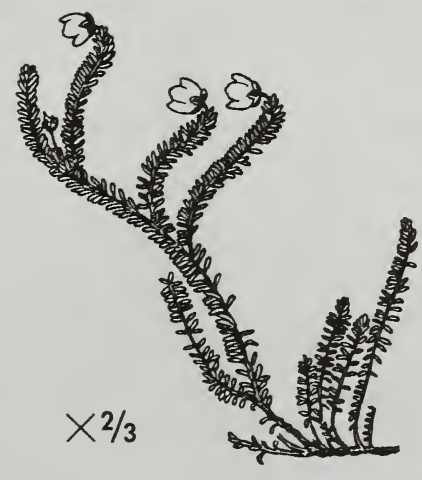


Cassiope tetragona

four-angled cassiope

HABIT: Dwarf shrub.

KEY CHARACTERS: Coarse, dark green, leaves in 4 rows, lance-shaped, deeply grooved and slightly hairy. Bell-shaped corolla.

HABITAT: Dry heaths and rocks on tundra or in the mountains, to at least 6500 feet. Common in the North.
CATE1 1

Ericaceae

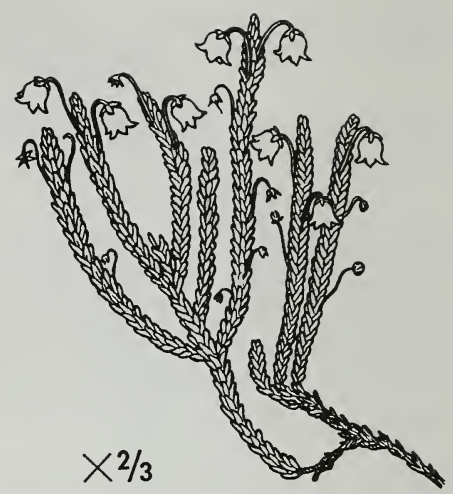

Cladothamnus pyrolaeflorus CLPY3 copperbush

Ericaceae

HABIT: Deciduous upright shrub, 11/2-41/2 feet.

KEY CHARACTERS: Leaves whorled, in clusters, light green above, white underneath, with pointed tip and nearly no petiole. Flowers copper colored, few, terminal, with long, curved style. Fruit $1 / 8-1 / 4$ inch diameter round capsules.

HABITAT: At and above treeline, and cold sites at lower elevations. Open forests, meadows, streambanks, openings.

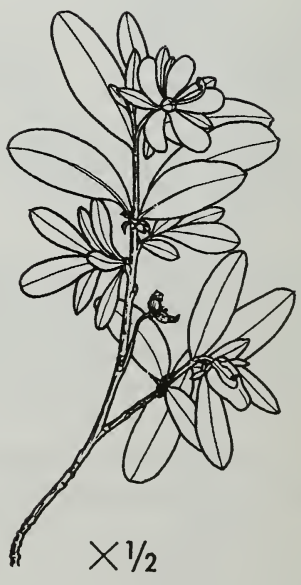

Generally with Mountain hemlock. 
Diapensia lapponica diapensia
DILA

Diapensiaceae

HABIT: Evergreen prostrate shrub, 1-3 inches.

KEY CHARACTERS: Leaves alternate, thick, fleshy, crowded and overlapping, with one vein. Flowers white, $3 / 4$ inch across, solitary, upright. Fruit $1 / 2$ inch diameter, eggshaped, upright capsules.

HABITAT: Rocky alpine areas.

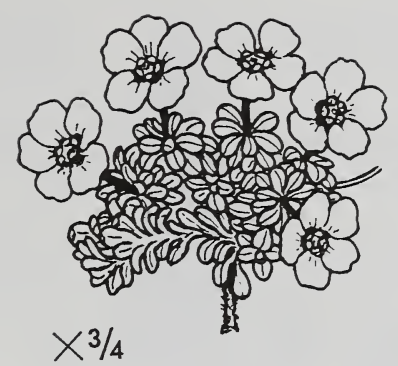

Dryas octopetala white mountain-avens
DROC

Rosaceae

HABIT: Evergreen, decumbent or mat-forming shrub, 1-5 inches.

KEY CHARACTERS: Leaves alternate, shiny green above, white woolly and with scales and hairs on midvein underneath, edges rolled under, with rounded teeth and sunken veins. Flowers white, solitary, on long, leafless stalks, stalks woolly, sepals with long, dark glands. Fruit nutlets with long plumes.

HABITAT: Forests, heaths, tundra.

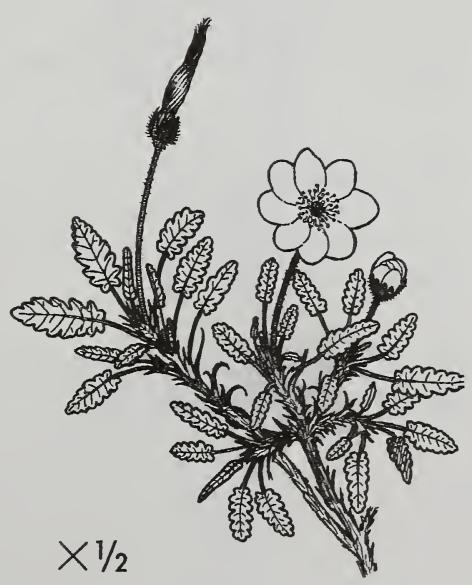


Echinopanax horridum devil's club

HABIT: Deciduous, with few long, prickly, decumbent branches, 2-10 feet.

KEY CHARACTERS: Leaves alternate, 1-2 feet long, 5-7 lobed, spiny. Branches with stout prickles. Flowers in terminal spike. Fruit red berries.

HABITAT: Moist forests, under alder, on good Sitka spruce sites, in subalpine with mountain hemlock.

Empetrum nigrum crowberry
ECHO2

Araliaceae
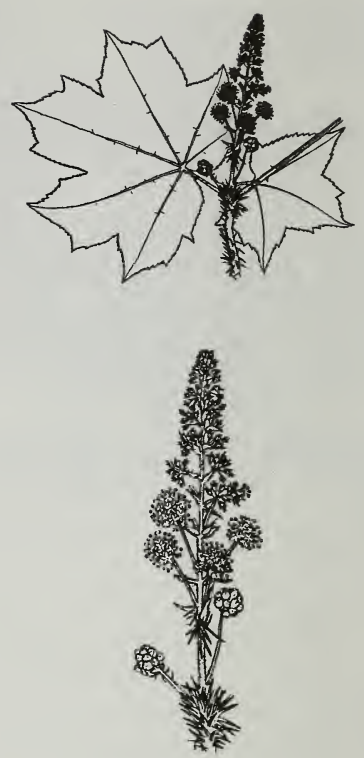

EMNI

Empetraceae

HABIT: Evergreen, mat-forming shrub, 3-6 inches.

KEY CHARACTERS: Leaves 4 in a whorl or alternate, needle-like, with white groove underneath. Flowers inconspicuous. Fruits berry-like, black.

HABITAT: Sea level to alpine. Forests, muskegs, rocky alpine slopes.

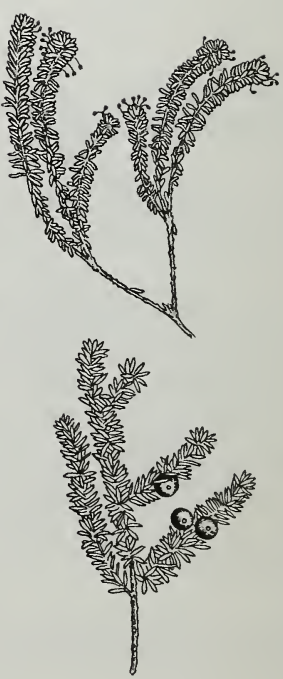


Juniperus communis

common juniper
JUCO6

Cupressaceae

HABIT: Evergreen spreading shrub, 1-2 feet.

KEY CHARACTERS: Needles in whorls of 3 , spreading at right angle from twig, with white band above. Cones berry-like, green ripening to black.

HABITAT: Dry slopes.

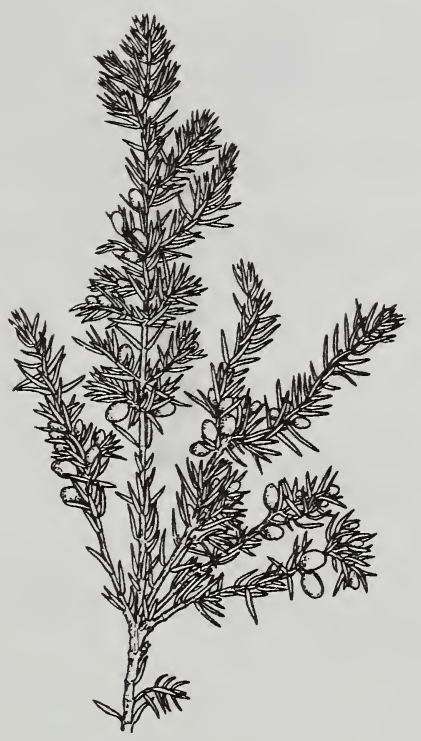

Ledum palustre

subsp. decumbens

LEPAD

narrow-leaf Labrador-tea Ericaceae

HABIT: Evergreen low shrub, 1-2 feet.

KEY CHARACTERS: Leaves alternate, shorter than 1 inch, less than 1/8 inch wide, with edges rolled under and reddish-brown woolly hairs underneath. Flowers white, $1 / 2$ inch across, in terminal clusters, with 10 stamens. Flower stalks with short white hairs. Fruit 1/8-1/4 inch long, oval capsules.

HABITAT: To 5400 feet. Black spruce forests, heaths, muskegs.

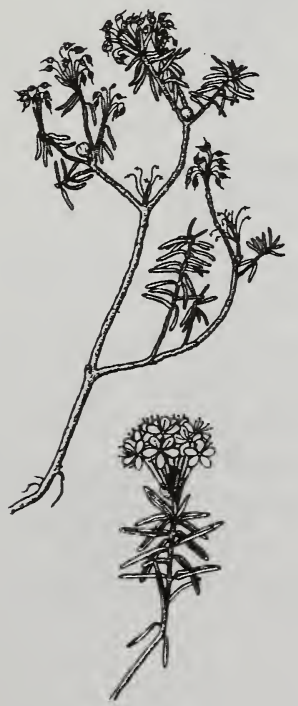


Ledum palustre

subsp. groenlandicum

Greenland Labrador-tea

LEPAG

Ericaceae

HABIT: Evergreen low shrub, to 3 feet.

KEY CHARACTERS: Leaves alternate, 1-2 inches long, greater than $1 / 8$ inch wide, fragrant, with edges rolled under and reddish-brown woolly hairs underneath.

Flowers white, $5 / 8$ inch across, in terminal clusters, fragrant, with 8 stamens. Flower stalks with reddish hairs. Fruit $1 / 4$ inch long capsules.

HABITAT: Black spruce forests, birch forests, with white spruce near treeline, muskegs.

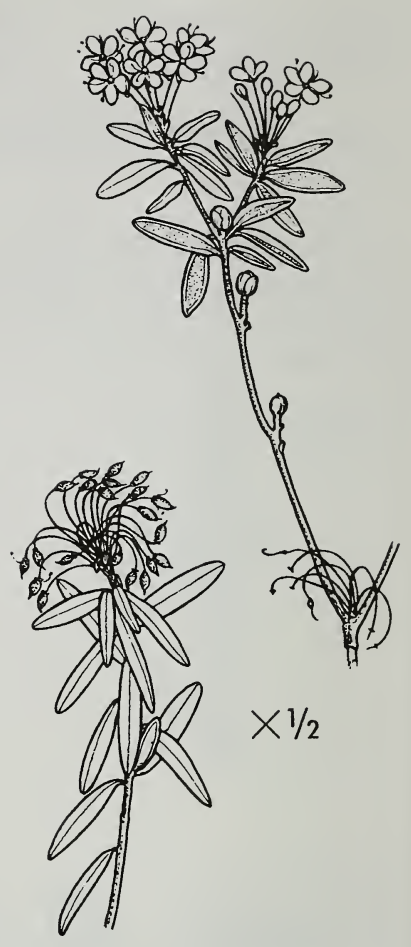

Linnaea borealis twinflower

LIBO3

Caprifoliaceae

HABIT: Evergreen, creeping, 1-4 inches.

KEY CHARACTERS: Leaves opposite, roundish, leathery, with 1-3 teeth on each side. Flowers white to rose, fragrant, nodding, in terminal pairs on upright stems. Fruits hairy.

HABITAT: Forests, heaths.

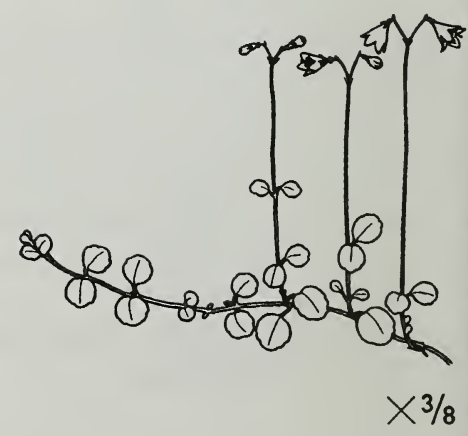


Loiseleuria procumbens alpine azalea
LOPR

Ericaceae

HABIT: Evergreen mat forming much branched shrub, 1-2 inches.

KEY CHARACTERS: Leaves opposite, white underneath, edges rolled under, leathery. Flowers pink, $1 / 8$ inch across, terminal, upright. Fruit $1 / 8$ inch diameter, round, upright, 2-3 parted capsules.

HABITAT: Well-drained acidic alpine tundra.

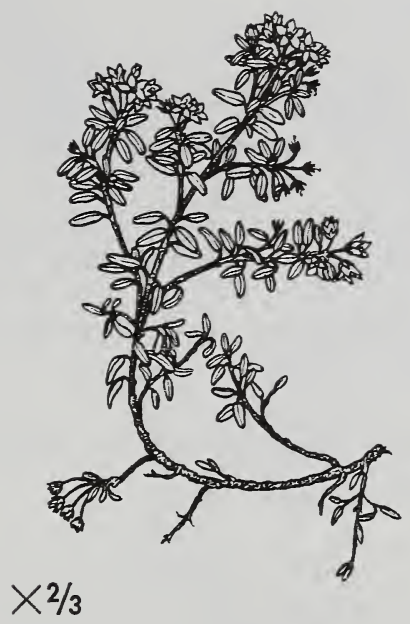

$\begin{array}{ll}\text { Luetkea pectinata } & \text { LUPE } \\ \text { luetkea } & \text { Rosaceae }\end{array}$

HABIT: Creeping shrub with upright leafy stems, 2-6 inches.

KEY CHARACTERS: Leaves crowded at base of stems, alternate along stem, fanshaped, with many narrow divisions.

Flowers white, in terminal cluster. Fruit pod-like.

HABITAT: Forests near treeline, alpine and subalpine meadows near snow.

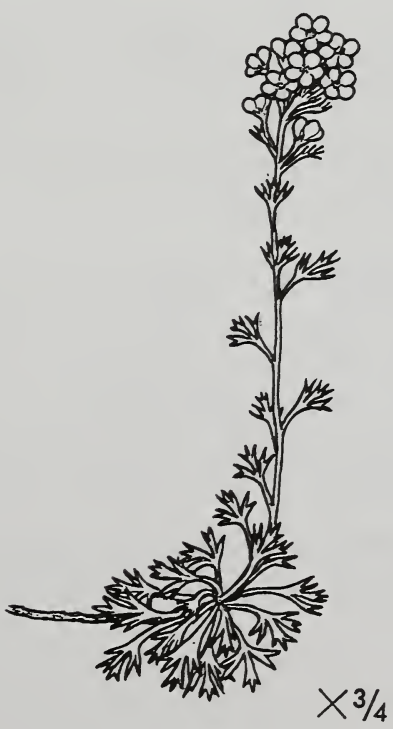


Malus fusca

Oregon crab apple

MAFU

Rosaceae

HABIT: Deciduous large shrub or small tree with several thorny stems, to 25 feet.

KEY CHARACTERS: Leaves alternate, toothed, pale and hairy underneath, often with 3 lobes. White or pink, few-flowered inflorescences. Fruits apple-like, yellow or red.

HABITAT: Moist forests, meadows, streambanks, beach forests.

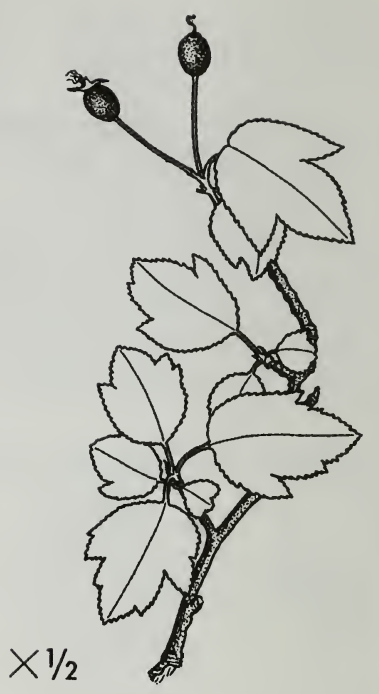

MEFE

Ericaceae
Menziesia ferruginea rusty menziesia
HABIT: Deciduous spreading to upright shrub. 3-10 feet.

KEY CHARACTERS: Leaves alternate, gray-green with brown hairs above, white with sticky hairs underneath. Twigs with odor when crushed. Flowers yellowish-red, several, terminal. Fruit $1 / 4$ inch long 4 -parted capsules, persistent through winter.

HABITAT: Moist forests, openings, under spruce-hemlock and spruce-birch.

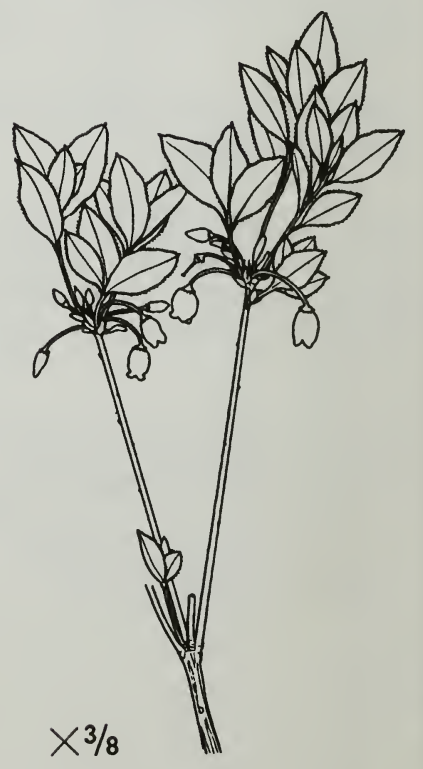


Myrica gale

sweetgale
MYGA

Myricaceae

HABIT: Deciduous low shrub, 1-4 feet.

KEY CHARACTERS: Leaves alternate, toothed toward tip, with yellow waxy glands and gray-green pubescence. Twigs with yellow resin dots. Flowers yellowish, inconspicuous, blooming before leaves appear. Fruit small, waxy, 2-winged, green nutlets.

HABITAT: Coastal meadows, muskegs, swamps, shallow water, tidal flats.

Oxycoccus microcarpus bog cranberry
OXMI3

Ericaceae

HABIT: Evergreen creeping vine-like shrub, 1-3 inches.

KEY CHARACTERS: Leaves alternate, $1 / 8-3 / 8$ inch long, dark green above, white

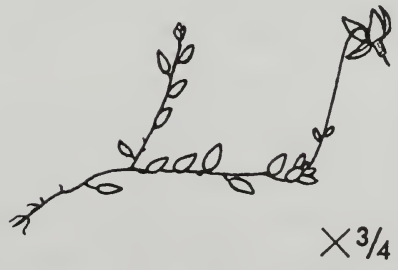
underneath, with edges rolled under. Twigs very slender. Flowers pink, solitary, on long, leafless stalks, nodding, with petals bent backward. Fruit red berries.

HABITAT: Muskegs, alpine meadows. 
Phyllodoce aleutica

Aleutian mountain heath

HABIT: Evergreen mat-forming shrub, 2-6 inches.

KEY CHARACTERS: Leaves crowded on upper 2-4 inches of stem, needle-like, toothed, with a pale groove and hairs underneath. Twigs with peg-like leaf scars. Flowers yellowish, several, terminal, may have glandular hairs. Fruit $3 / 8$ inch long, oval, 5-parted capsules.

HABITAT: Sea level to alpine. Moraines and outwash at sea level, heaths, protected slopes adjacent to snow fields.
Potentilla fruticosa shrubby cinquefoil
POFR4

Rosaceae
HABiT: Deciduous spreading to upright much-branched shrub, 1-4 feet.

KEY CHARACTERS: Leaves alternate, with 3-7 leaflets, green above, grayish and silvery hairy underneath. Flowers yellow, $3 / 4-1 \frac{1}{4}$ inches across, upright, solitary, from leaf bases or in terminal clusters. Fruit white-haired achenes.

HABITAT: To 4500 feet. Forests, muskegs, wet soil, rocky slopes.
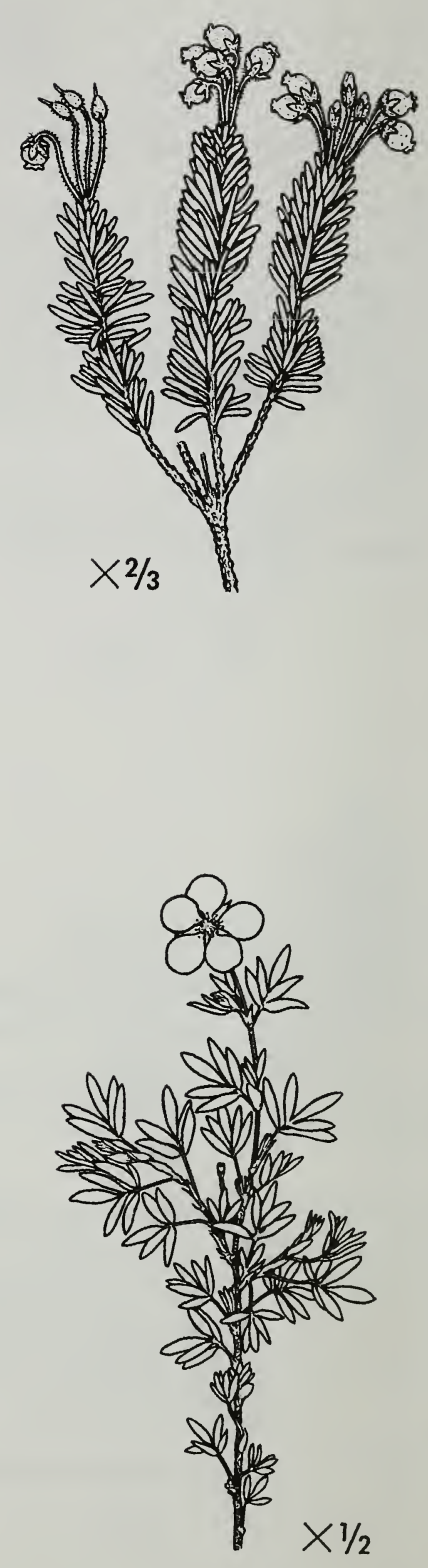
Ribes bracteosum

stink currant
RIBR

Saxifragaceae

HABIT: Deciduous ascending or upright shrub, 3-6 feet.

KEY CHARACTERS: Leaves alternate, 5-7 lobed, singly or doubly toothed.

Skunky odor to plant. Flowers white, greenish, or purplish, with hairy and glandular ovary. Racemes upright, 3-12 inches long. Fruit glandular, disagreeable-tasting, black berries.

HABITAT: Forests, streambanks, with alder in spruce-hemlock forests.

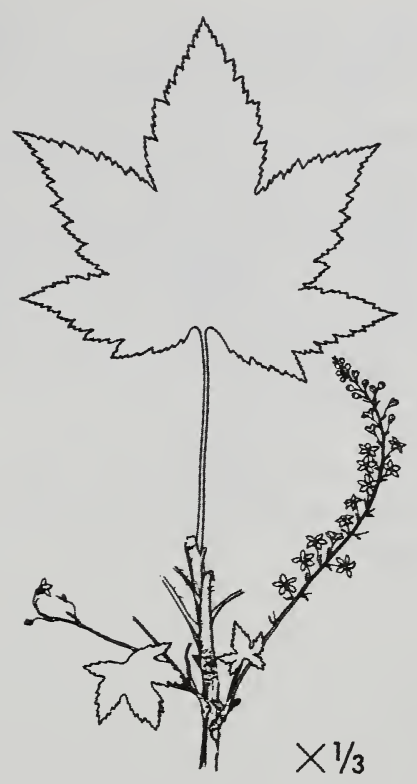

Ribes glandulosum

RIGL

skunk currant

Saxifragaceae

HABIT: Deciduous, ascending shrub.

KEY CHARACTERS: Smooth, deeply heart-shaped leaves, 5-7 lobed, sharp-pointed, doubly serrate. Whitish or pink flowers. Red fruit, with the odor of skunk.

HABITAT: Woods, rocky slopes in the lowlands.

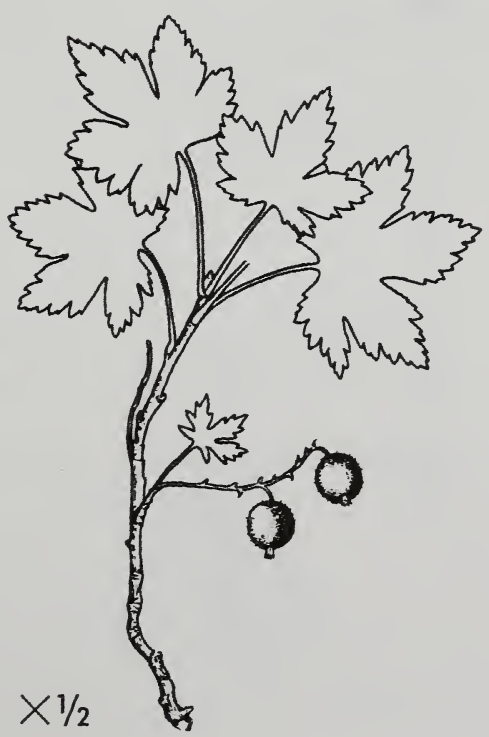


Ribes hudsonianum

northern black currant

RIHU

Saxifragaceae

HABIT: Deciduous upright shrub, 3-6 feet.

KEY CHARACTERS: Leaves alternate, 3-5 lobed, toothed. Flowers white with yellow-dotted ovary. Racemes ascending or spreading, 1-2 $\frac{1}{2}$ inches long. Fruit smooth or glandular, bitter but edible black berries, with bloom.

HABITAT: Moist forests, streambanks, at treeline with alder, with spruce, birch, and aspen.
RILA

Saxifragaceae

Ribes lacustre
bristly black currant

HABIT: Deciduous spreading to upright spiny shrub, 2-4 feet.

KEY CHARACTERS: Leaves alternate, with 3-5 lobes, the lobes again lobed or toothed. Twigs spiny. Flowers pink, spreading or drooping racemes. Fruit palatable dark purple berries with gland-tipped hairs.

HABITAT: Moist forests, streambanks, with white spruce and Sitka spruce.
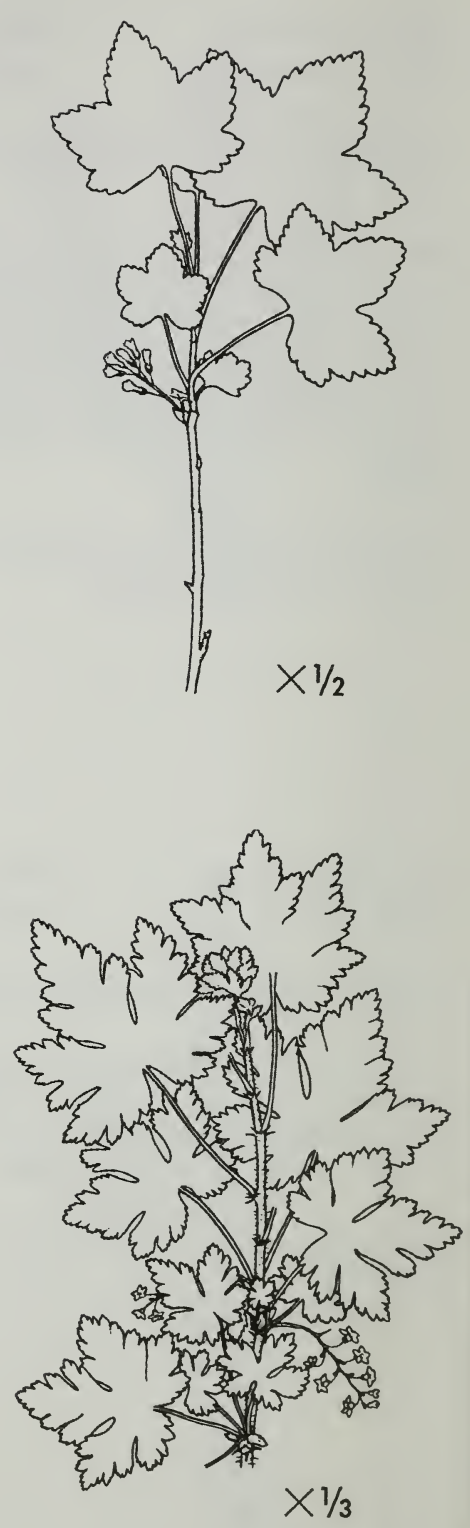
Ribes laxiflorum

trailing black currant
RILA3

Saxifragaceae

HABIT: Deciduous decumbent to upright shrub, sometimes vine-like, to 2 feet.

KEY CHARACTERS: Leaves alternate, blades $2 \frac{1}{2}-3$ inches-long, doubly toothed, with 5 lobes. Flowers red to purplish, ovary with hairs and stalked glands, sepals hairy on back. Racemes ascending or upright, 4-6 inches long, with 6-20 flowers. Fruit foul smelling, edible, purplish-black berries, with bloom and stalked glands.

HABITAT: Moist forests, open meadows, wet areas, spruce-hemlock forests.

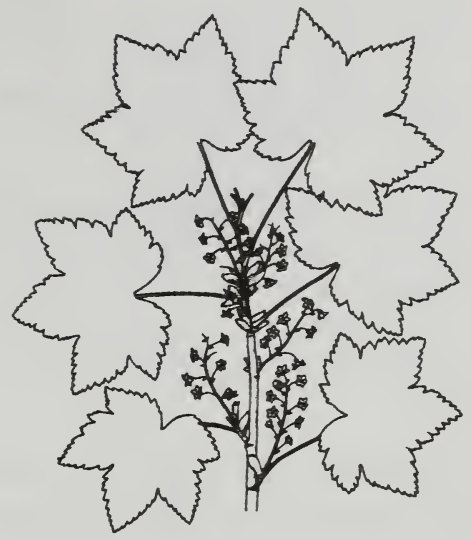

$\times 1 / 4$
Ribes triste

northern red currant

HABIT: Deciduous prostrate to erect shrub, 2-3 feet.

KEY CHARACTERS: Leaves alternate, with 3-5 lobes, toothed. Flowers purple, with smooth ovary. Racemes 2-4 inches long. Fruit ovoid, smooth, palatable, bright red, translucent berries.

HABITAT: Lowlands to treeline. Spruce forests, streambanks, wet meadows.

RITR

Saxifragaceae

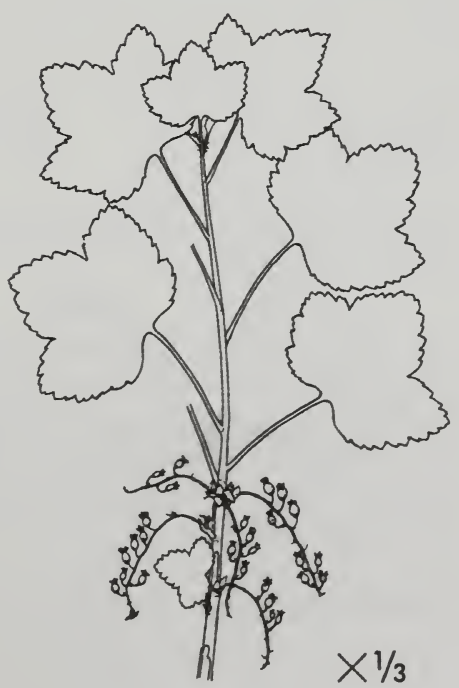


Rosa acicularis prickly rose
ROAC

Rosaceae

HABIT: Deciduous much-branched spiny shrub, 1-4 feet.

KEY CHARACTERS: Leaves alternate, toothed, with 3-7 leaflets. Twigs and branches with prickles and spines. Flowers rose to pink, 11/2-21/4 inches across, usually solitary, terminal. Fruit dark red to purplish hips.

HABITAT: Open forests, meadows, thick$\times 1 / 3$
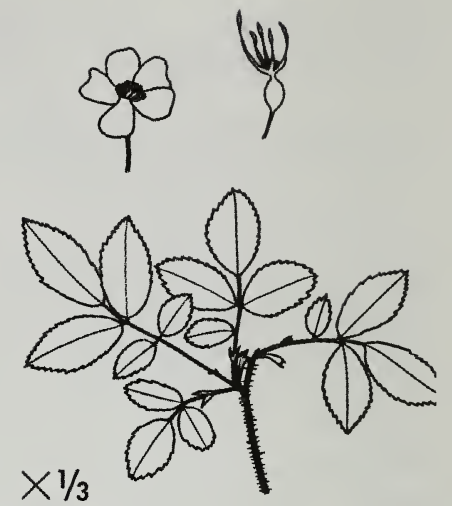
ets, muskegs, with aspen on burns.

Rubus idaeus raspberry
RUID

Rosaceae

HABIT: Deciduous upright prickly biennial shrub, 2-4 feet.

KEY CHARACTERS: Leaves alternate, whitish underneath, toothed, with 3-5 leaflets. Twigs and branches with prickles. Flowers white, $1 / 2$ inch across, $1-4$ in raceme, with sepals bent backward. Fruit red, $3 / 4$ inch long.

HABITAT: Forests, thickets.

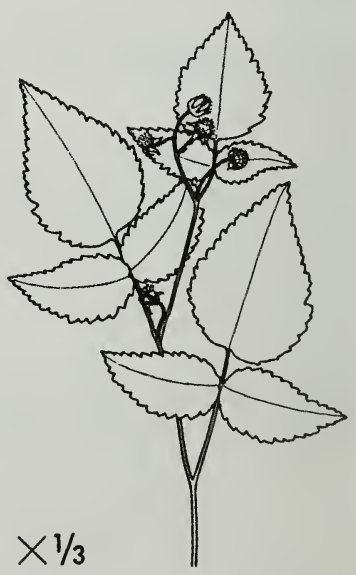




\section{Rubus spectabilis} salmonberry

RUSP

Rosaceae

HABIT: Deciduous upright somewhat prickly biennial shrub, 2-7 feet.

KEY CHARACTERS: Leaves alternate, usually with 3 leaflets, the leaflets toothed to nearly lobed. Flowers pink, 11/2 inches across, solitary or 2 . Fruit red or yellow, 1 inch long.

HABITAT: To lower alpine. Moist forests, streambanks, avalanche chutes, sideslopes, and ravines.

Salix alaxensis feltleaf willow

SAAL

Salicaceae

HABIT: Shrub or small tree, up to $20-25$ feet.

KEY CHARACTERS: Young twigs woolly. Leaves tear-shaped with dense white felt beneath. Stout, erect catkins.

HABITAT: Common along creeks and rivers, to at least 6000 feet.
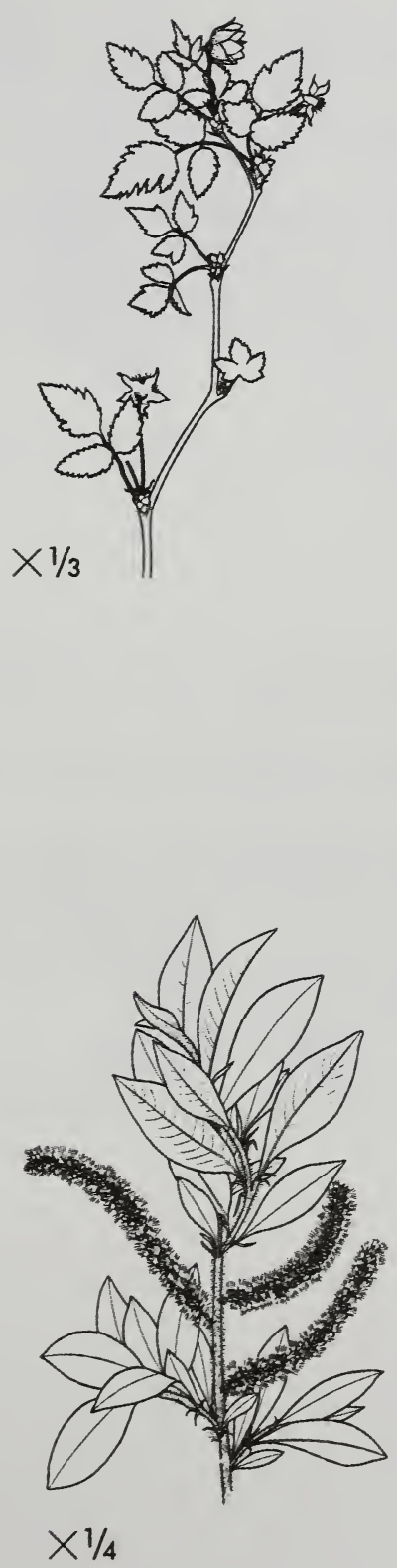
Salix arctica arctic willow
SAAR4

Salicaceae

HABIT: Dwarf shrub.

KEY CHARACTERS: Thick, smooth branches. Leaves ovate, entire, dark green above, paler beneath, slightly hairy when young but later smooth. Catkins about 1 inch long.

HABITAT: Dry tundra, mostly in mountains.

Salix barclayi

Barclay willow
SABA3

Salicaceae

HABIT: Deciduous shrub, 6-9 feet.

KEY CHARACTERS: Leaves alternate, white underneath, often toothed, turning black when dry. Buds without bud scales. Inflorescence a catkin. Fruit hairless capsules.

HABITAT: Forest openings, streambanks, wet areas, alpine and subalpine slopes.
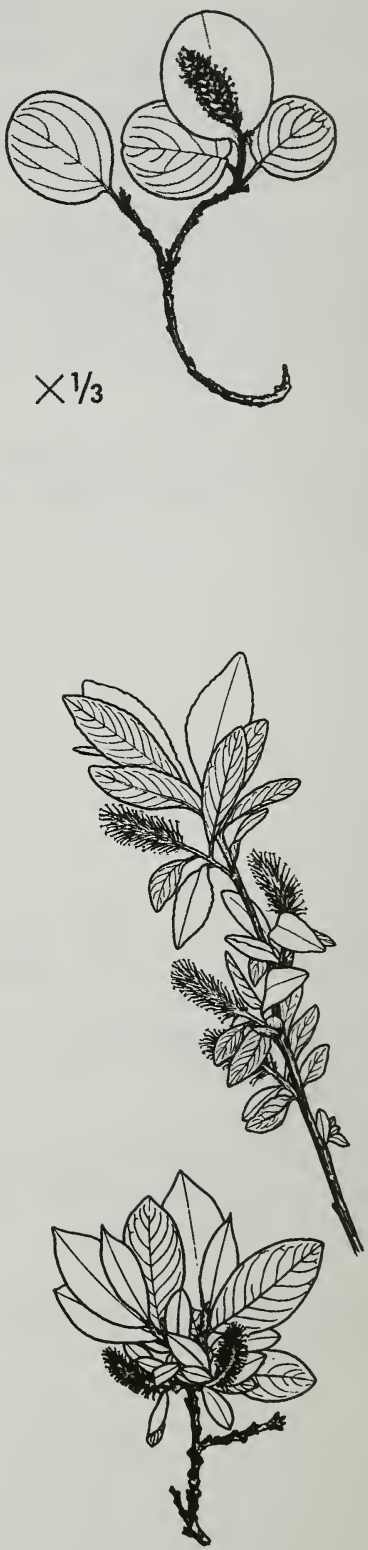
Salix commutata

undergreen willow
$\mathrm{SACO} 2$

Salicaceae

HABIT: Shrub up to 6 feet.

KEY CHARACTERS: Dense gray hair on young twigs. Leaves elliptical, pointed at tips, with dense gray hair on both sides when young, becoming smooth with age.

HABITAT: Alpine meadows and wet areas.

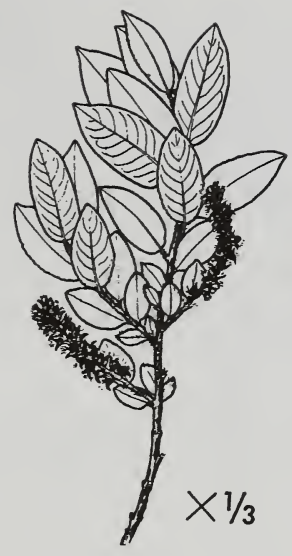

Salix depressa subsp. rostrata Bebb willow

SADER

Salicaceae

HABIT: Deciduous large shrub or small tree, to 30 feet.

KEY CHARACTERS: Leaves alternate, $1-3 \frac{1}{2}$ inches long, dull gray above and gray or whitish underneath, pointed at base, nearly toothless, with hairs on both sides. Twigs leave branches nearly at right angles. Buds with no bud scales. Inflorescences 13 inch long catkins with short, leafy stalks appearing before or with the leaves. Fruit

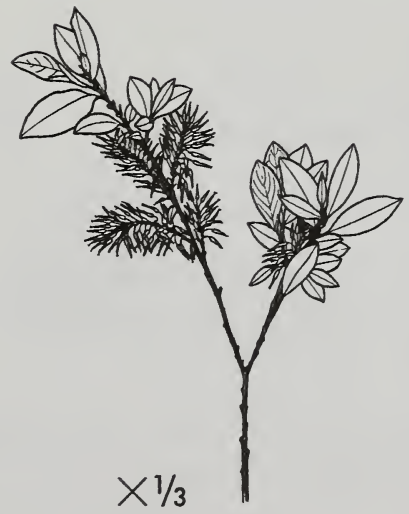
hairy capsules on 1/8- 3/16 inch long stalks.

HABITAT: To subalpine. Dry areas in forests. 
Salix hookeriana

Hooker willow

\section{SAHO}

Salicaceae

Not in Hultén (1968). See Welch (1974).

HABIT: Deciduous shrub, 2-15 feet.

KEY CHARACTERS: Leaves alternate, hairy on both surfaces, branches very brittle. Inflorescence a catkin.

HABITAT: Stabilized sand dunes, wet meadows near the coast, beach ridges.

Salix myrtillifolia

low blueberry willow
SAMY

Salicaceae

HABIT: Deciduous low shrub, to 3 feet.

KEY CHARACTERS: Leaves alternate, green on both surfaces, often drying brownish.

HABITAT: Muskegs, fens, wet lake margins and river banks.

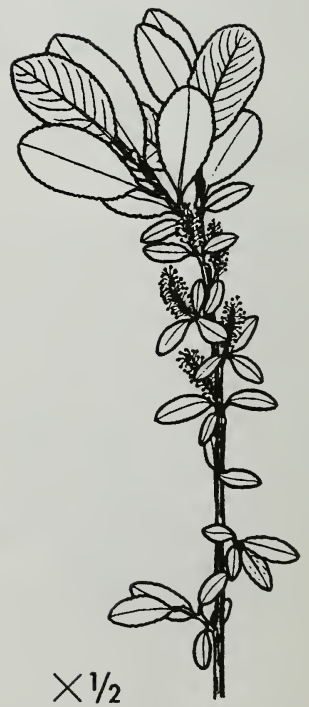


Salix pulchra

tealeaf willow
SAPU15

Salicaceae

HABIT: Deciduous shrub, to 6 feet.

KEY CHARACTERS: Leaves alternate, green above, whitish below. Previous years leaves often persisting as brown, withered remains.

HABITAT: Alpine tundra, coniferous woodlands, shrub thickets along streams and lakes.

Salix reticulata

netleaf willow
SARE2

Salicaceae

HABIT: Deciduous prostrate shrub, 2-3 inches.

KEY CHARACTERS: Leaves alternate, dark green, leathery, distinctly net-veined, with edges rolled under. Inflorescence an upright catkin, to 2 inches long. Fruit capsules with white hairs.

HABITAT: Dry and moist tundra.

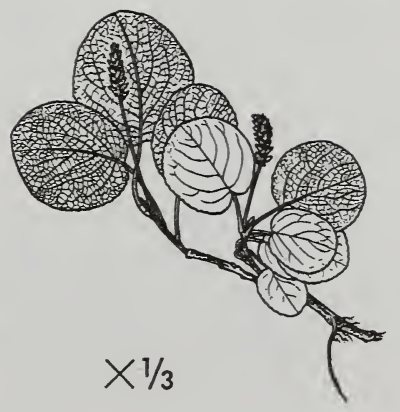


Salix rotundifolia

least willow
SARO2

Salicaceae

HABIT: Dwarf shrub, forming mats.

KEY CHARACTERS: Very thin, slightly hairy annual shoots. Small, leathery, round or ovate leaves, entire with lateral veins raised on both sides. Very short catkins, few-flowered.

HABITAT: Arctic and alpine lichen tundra, rocky places, to at least 6500 feet.

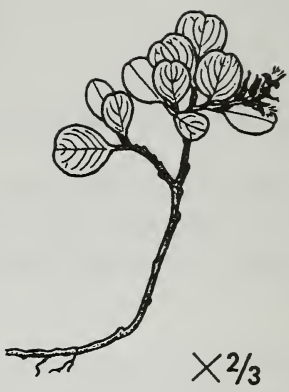

Salix sitchensis

Sitka willow
SASI2

Salicaceae

HABIT: Shrub, up to 30 feet with trunk 12 inches in diameter.

KEY CHARACTERS: Twigs hairy when young, more or less smooth with age.

Leaves oblong or tear-shaped, with silky hairs. Long, slender catkins, densely flowered.

HABITAT: Along streams and shores and wet areas.

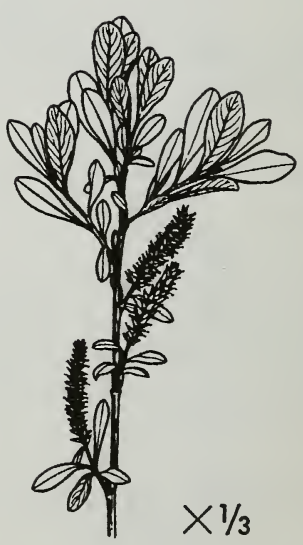


Sambucus racemosa elderberry
SARA2

Caprifoliaceae

HABIT: Deciduous clump-forming shrub, 6-12 feet.

KEY CHARACTERS: Leaves opposite, 5-10 inches long, toothed, hairy underneath, with 5-7 leaflets. Unpleasant odor to crushed leaves or twigs. Flowers small, white, in a terminal pyramidal inflorescences. Fruit small red drupes. Bark brown and warty.

HABITAT: Forests, thickets with alder or willow, streambanks, subalpine meadows.

Shepherdia canadensis buffaloberry
SHCA

Elaeagnaceae

HABIT: Deciduous shrub, to 6 feet.

KEY CHARACTERS: Leaves opposite, green above, with reddish scales underneath. Twigs with brownish scales. Flowers small, yellowish or brownish. Fruit berrylike, yellow or red, $1 / 4$ inch long.

HABITAT: To 3600 feet. Well-drained forests, gravel bars, with aspen on burns.
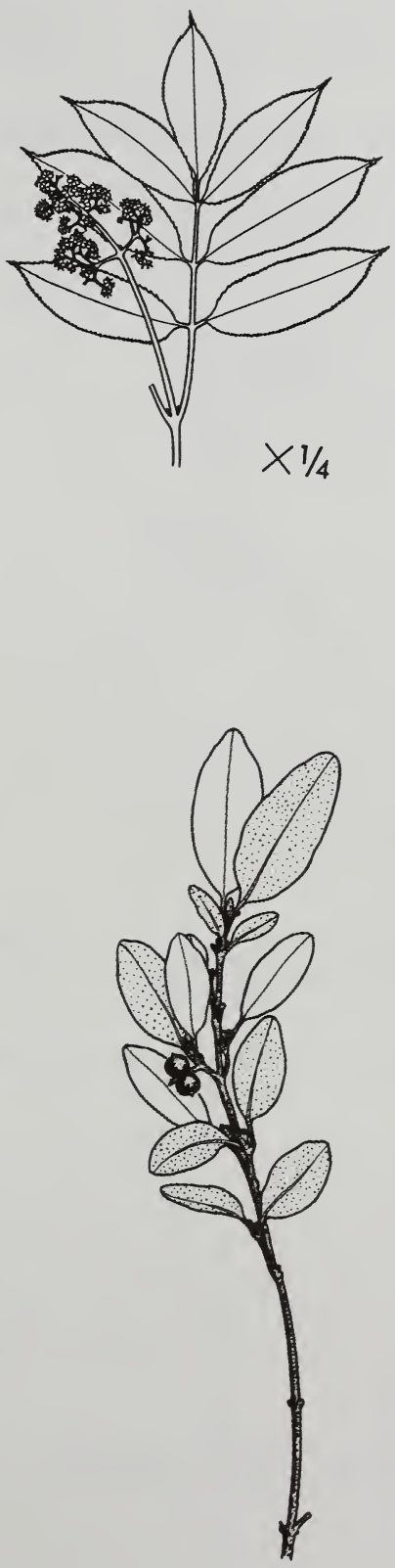
Sibbaldia procumbens

creeping sibbaldia
SIPR

Rosaceae

HABIT: Mat forming, 2-6 inches.

KEY CHARACTERS: Leaves with 3 leaflets, 3-toothed at apex, hairy on both surfaces. Flowers inconspicuous with pale yellow petals.

HABITAT: Alpine tundra, open woods in the subalpine zone.

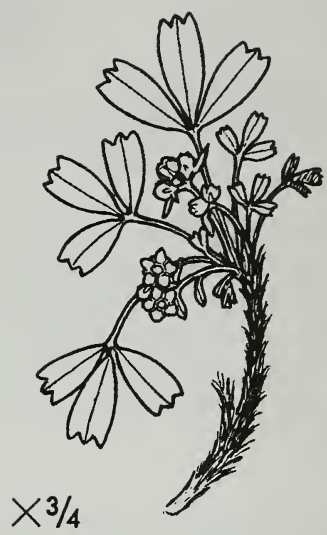

Sorbus scopulina western mountain-ash

\section{SOSC2}

Rosaceae

HABIT: Deciduous many-stemmed shrub, 3-13 feet, or small tree to 20 feet.

KEY CHARACTERS: Leaves alternate, singly or doubly toothed, pointy at tip, with 11-13 leaflets. Buds sticky. Buds and twigs with white hairs. Flowers white, in terminal, rounded inflorescences. Fruit applelike, orange to red, less than $3 / 8$ inch in diameter.

HABITAT: Forests, subalpine.

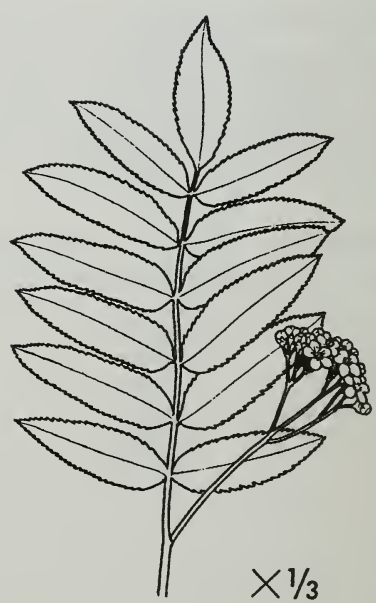


Sorbus sitchensis

Sitka mountain-ash
SOSI2

Rosaceae

HABIT: Deciduous shrub, 4-8 feet, or small tree, to 20 feet.

KEY CHARACTERS: Leaves alternate, toothed, rounded at tip, with 7-11 leaflets. Buds and twigs with brown hairs. Twigs with odor and bitter taste of cherry. Flowers white, fragrant, in terminal rounded inflorescences. Fruits apple-like, red with bluish bloom, $1 / 2$ inch in diameter.

HABITAT: Sea level to timberline.

Spiraea beauverdiana

Beauverd spiraea
SPBE

Rosaceae

HABIT: Deciduous low to midsize manybranched shrub, 1-2 feet.

KEY CHARACTERS: Leaves alternate, toothed especially toward tip. Young branches reddish-brown. Flowers white or pink in a flat-topped or hemispheric inflorescence. Fruit pod-like, persistent through winter.

HABITAT: Low elevation to alpine.

Forests, meadows, black spruce muskegs, tundra.
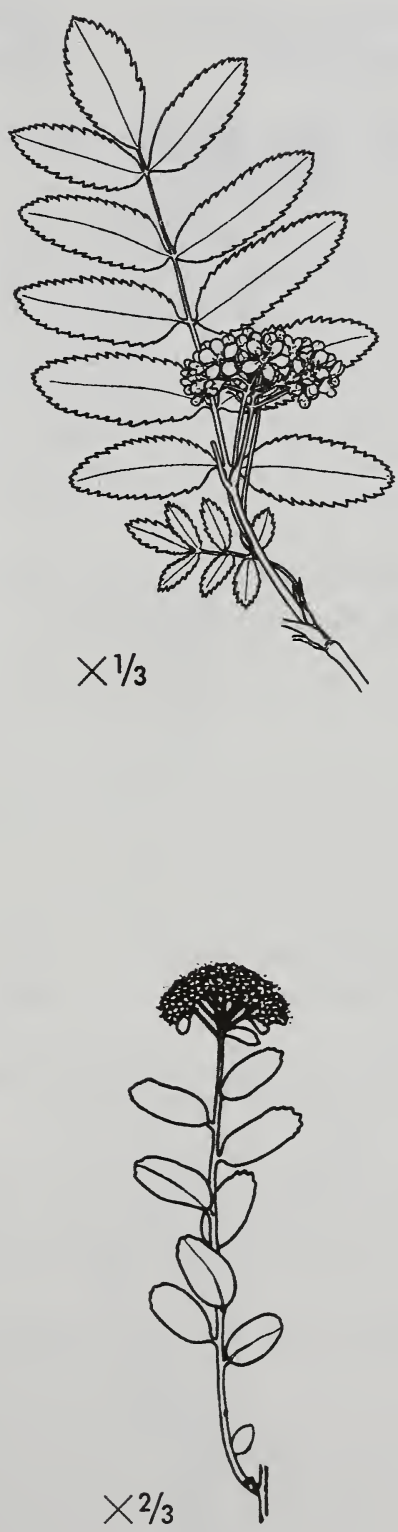
Vaccinium alaskensis

Alaska blueberry

HABIT: Deciduous spreading to upright shrub, up to 6 feet.

KEY CHARACTERS: Leaves alternate, with small teeth in lower half or no teeth, glands on midvein underneath. Flowers pink, solitary, from leaf bases, appearing with leaves or after leaves partially developed. Fruit blue-black berries, usually without bloom. Fruit stalk straight, enlarged below fruit, often longer than $3 / 8$ inch.

HABITAT: Spruce-hemlock forests on the coast, especially in openings.

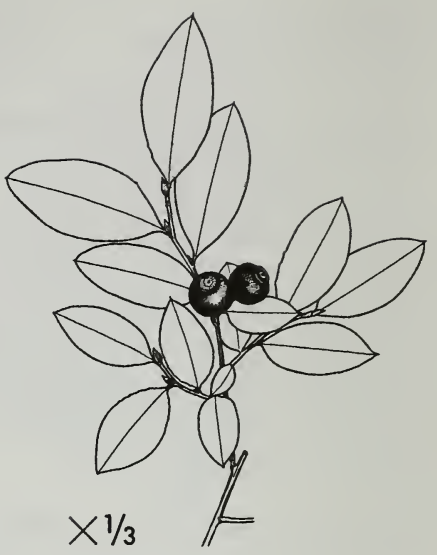

Vaccinium caespitosum VACA13 dwarf blueberry

Ericaceae

HABIT: Deciduous spreading muchbranched shrub, 4-16 inches.

KEY CHARACTERS: Leaves alternate, toothed. Flowers pink to white, solitary, from leaf bases. Fruit blue to black berries.

HABITAT: Open forests, muskegs.

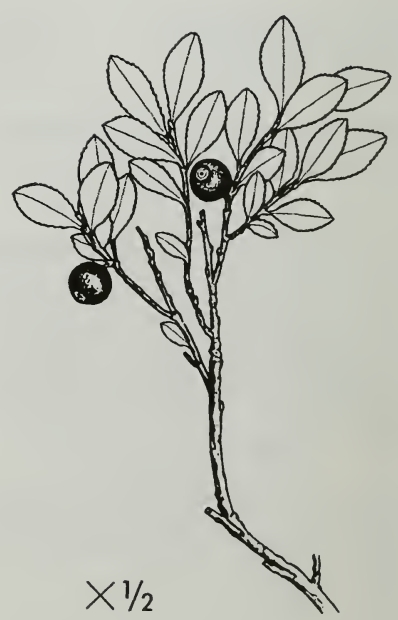


Vaccinium ovalifolium early blueberry
VAOV

Ericaceae

HABIT: Deciduous spreading shrub, to 5 feet.

KEY CHARACTERS: Leaves alternate, usually with no teeth or glands. Largest leaves at end of twigs. Flowers pink, solitary, from leaf bases, longer than broad, appearing before or with leaves. Fruit bluish or blue-black berries, with whitish bloom. Fruit stalk curved, not enlarged below fruit, usually shorter than $3 / 8$ inch.

HABITAT: To subalpine. Forests, muskegs, openings.

Vaccinium uliginosum bog blueberry
VAUL

Ericaceae

HABIT: Deciduous prostrate to upright much-branched shrub, 8-16 inches.

KEY CHARACTERS: Leaves alternate, pale and distinctly veined underneath, leathery. Flowers pink, terminal or from leaf bases. Fruit blue-black berries with bluish bloom.

HABITAT: Open forests, heaths, muskegs.
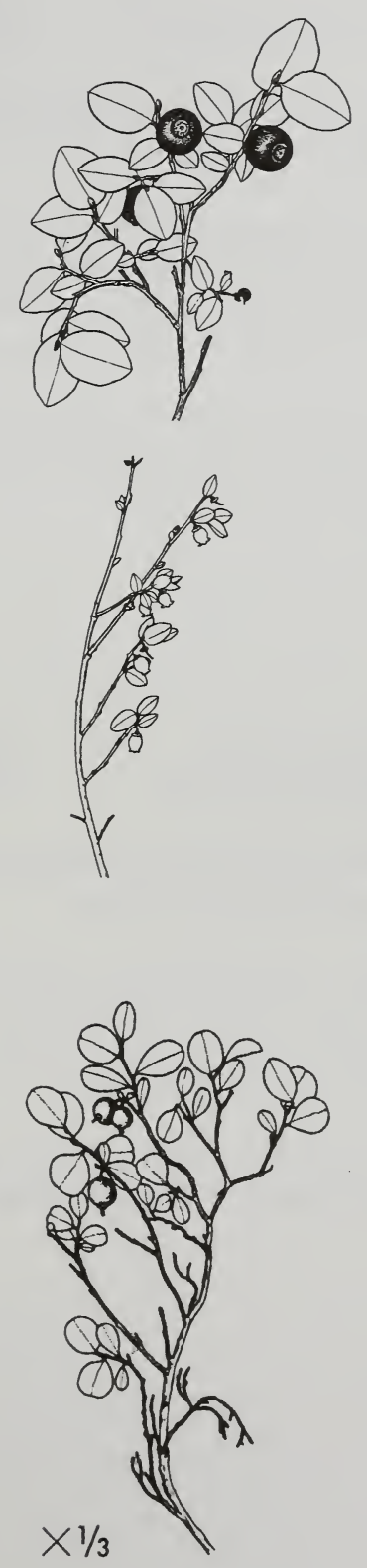
Vaccinium vitis-idaea lowbush cranberry
VAVI

Ericaceae

HABIT: Evergreen creeping mat-forming shrub, 2-6 inches.

KEY CHARACTERS: Leaves alternate, edges slightly rolled under, shiny, leathery, with dark dots and brown hairs underneath. Flowers pink, terminal. Fruit red berries.

HABITAT: Spruce and birch forests, muskegs, tundra, dry alpine slopes, acidic poorly drained soil.

VIED

Viburnum edule highbush cranberry

Caprifoliaceae

HABIT: Deciduous ascending manystemmed shrub, 2-8 feet.

KEY CHARACTERS: Leaves opposite, 3-lobed, toothed. Buds dark red-brown. Flowers white, in dense terminal clusters. Fruit red-orange drupes.

HABITAT: Forests, thickets, muskegs, streambanks, beaches, tidal meadows.
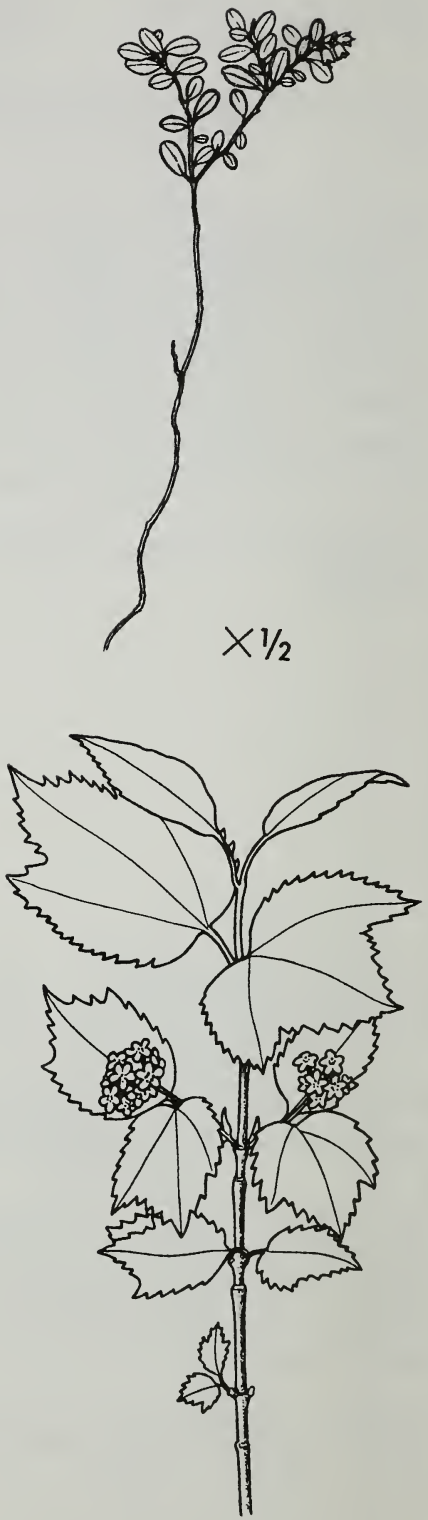


\section{FORBS}


Achillea borealis

yarrow
$\mathrm{ACBO}$

Compositae

HABIT: To 3 feet.

KEY CHARACTERS: Leaves alternate, 2-3 times pinnately divided, woolly, fragrant. Stem hairy. Flowers white, in several terminal clusters. Fruit hairless achenes.

HABITAT: To 5400 feet. Forests, meadows, sandy slopes, beaches.

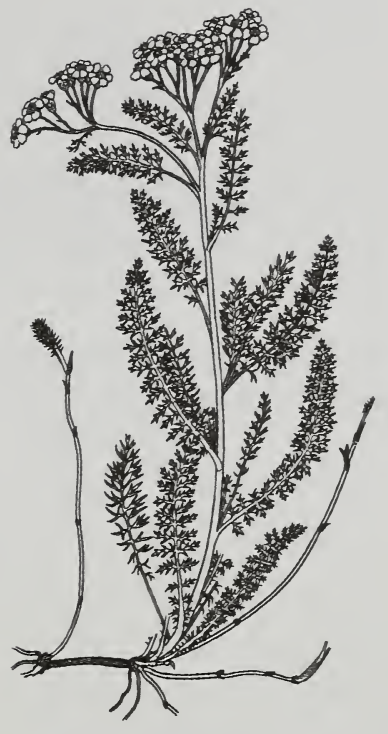

Aconitum delphinifolium ACDE2 monkshood Ranunculaceae

HABIT: Thin, straight, to 3-50 inches.

KEY CHARACTERS: Leaves alternate, rounded in outline, palmately 5-lobed, hairless, few. Flowers dark blue, hooded, in few-flowered terminal raceme. Fruit follicles. Poisonous.

HABITAT: To alpine. Meadows, streambanks.

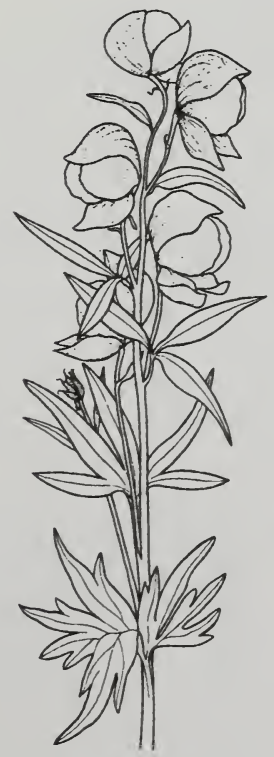


Actaea rubra

baneberry
ACRU2

Ranunculaceae

HABIT: Branched, 1-3 feet.

KEY CHARACTERS: Leaves alternate, ternate, toothed. No basal leaves. Flowers white, in a terminal raceme. Fruit red or white pea-sized berries.

HABITAT: Forests, streambanks, open slopes, beaches.

Anemone narcissiflora narcissus anemone

HABIT: 2-25 inches.

KEY CHARACTERS: Basal leaves rounded in outline, palmately compound, the leaflets with many divisions. Stem leaves whorled. Stems and leaves often hairy. Flowers large, white to creamy white, few to several per stem, terminal. Fruit hairless achenes.

HABITAT: Meadows, snowbeds in tundra.
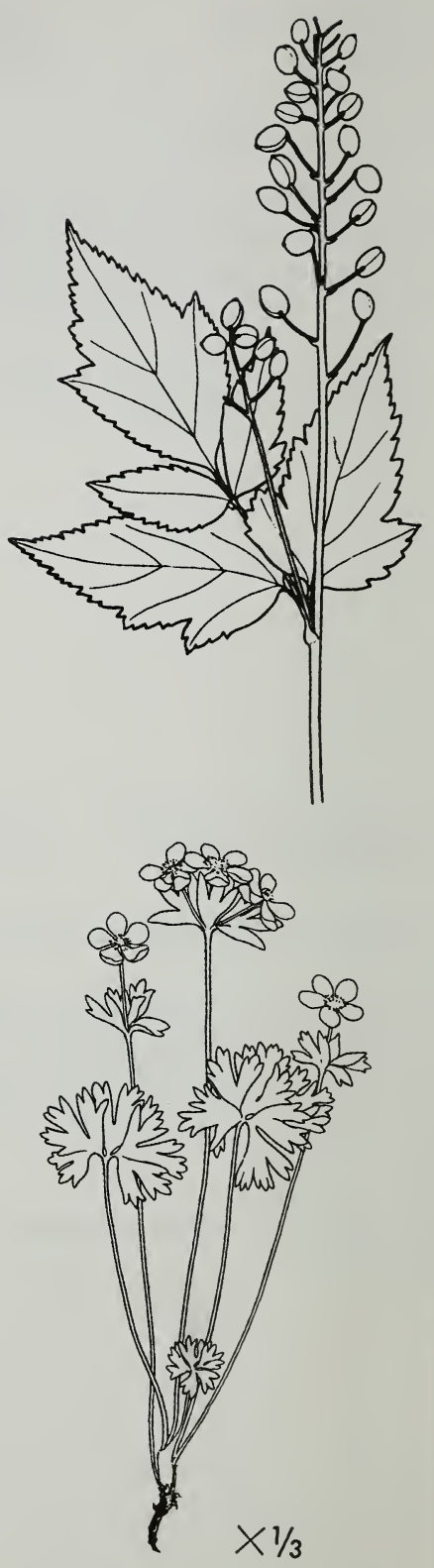
Anemone richardsonii

yellow anemone

HABIT: 2-12 inches.

KEY CHARACTERS: Leaves from rhizome, rounded, palmately 3-lobed, the lobes divided and toothed. Stem leaves in single whorl, with no petiole. Flowers yellow, solitary, terminal. Fruit hairless achenes with very long beak. Poisonous.

HABITAT: Meadows, snowbeds.
ANRI

Ranunculaceae

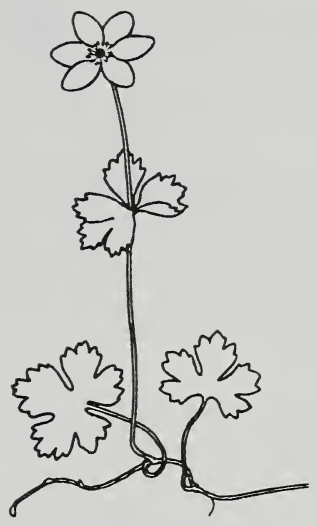

$\times 1 / 2$

Angelica genuflexa bent-leaved angelica
ANGE2

Umbelliferae

HABIT: $2-4 \frac{1}{2}$ feet.

KEY CHARACTERS: Stem leaves alternate, ternate to bipinnate, toothed, bending downward, with hairy edges and viens.

Stem hollow, often purple. Flowers white to pinkish, in terminal umbel. Fruits with lateral wings.

HABITAT: Meadows, swamps, streambanks, wet areas, beaches.

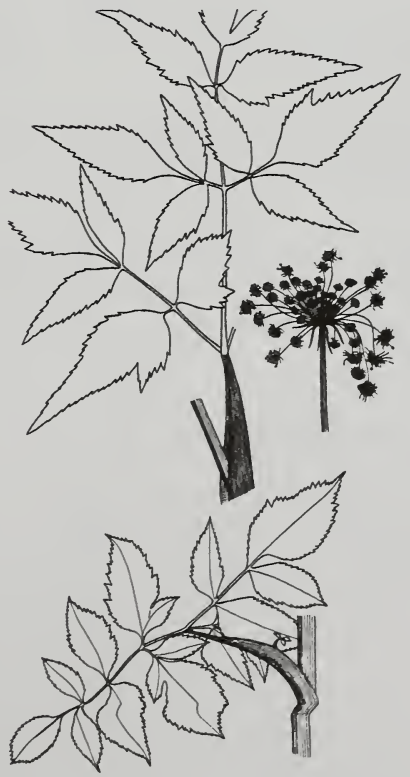


Angelica lucida

seacoast angelica
ANLU

Umbelliferae

HABIT: To 3 feet.

KEY CHARACTERS: Stem leaves alternate, 2-3 times ternate, toothed, with inflated petiole. Flowers greenish-white, in terminal umbel. Fruits with all ribs narrowly winged.

HABITAT: Meadows, thickets, wet areas, beaches.

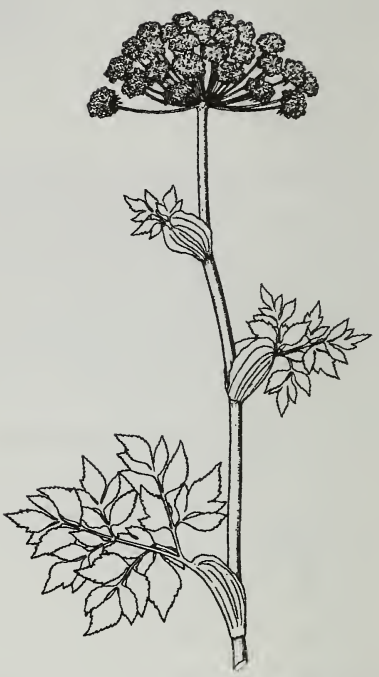

Antennaria monocephala ANMO9 pygmy pussytoes Compositae

HABIT: Mat forming, with stolons, 1-6 inches tall.

KEY CHARACTERS: Basal leaves 1/5-4/5 inches long, grayish hairy to hairless below, mostly hairless above. Flower heads solitary.

HABITAT: Alpine tundra.

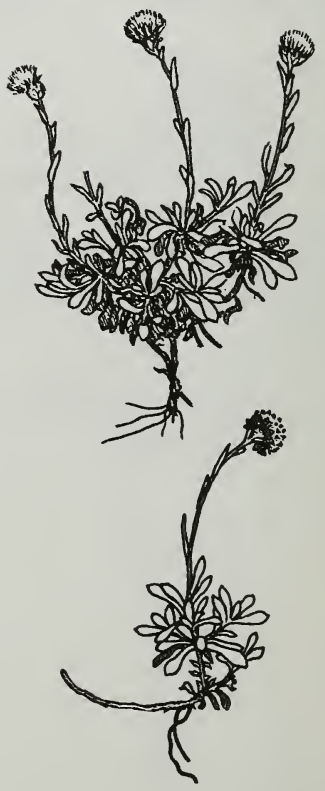


Apargidium boreale

apargidium
APBO

Compositae

HABIT: 4-20 inches.

KEY CHARACTERS: Leaves 2-10 inches long, $1 / 10-1 / 2$ inches wide. Petals yellow, often drying whitish or pinkish.

HABITAT: Wet meadows.

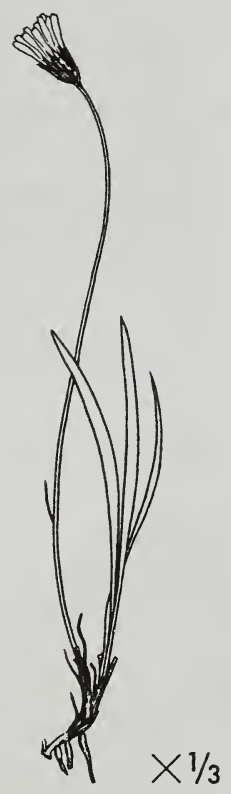

Aquilegia formosa

western columbine
AQFO

Ranunculaceae

HABIT: Branched, 1-3 feet.

KEY CHARACTERS: Leaves mostly basal, white underneath, twice ternate. Stem leaves alternate. Flowers with red sepals and spurs with yellow petals. Flowers 2-4 per stem, terminal, nodding. Fruit hairy follicles.

HABITAT: Moist forests, meadows, open areas, beaches.

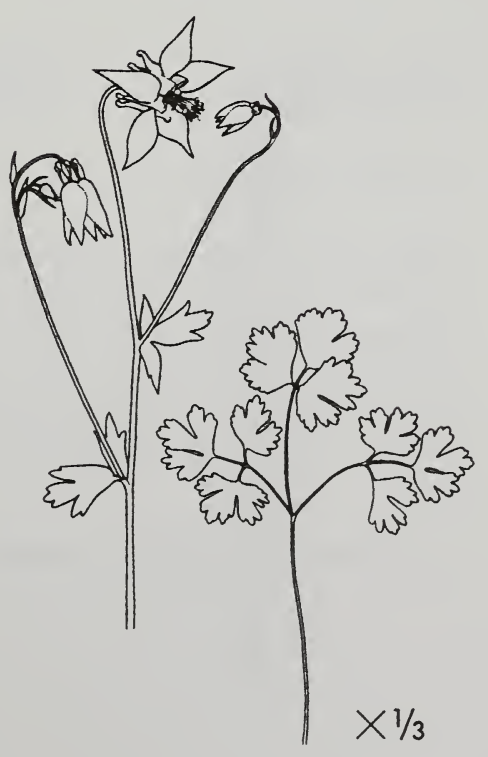


Arabis lyrata

lyreleaved rockcress
ARLY2

Cruciferae

HABIT: Branched, to 8 inches.

KEY CHARACTERS: Basal leaves reddish-purple, lyre-shaped to pinnately divided. Stem leaves alternate, dull purple, pinnately divided to linear. Flowers white or pink, few to several in terminal raceme. Fruit hairless capsules (siliques).

HABITAT: Moist stony places, scree slopes.

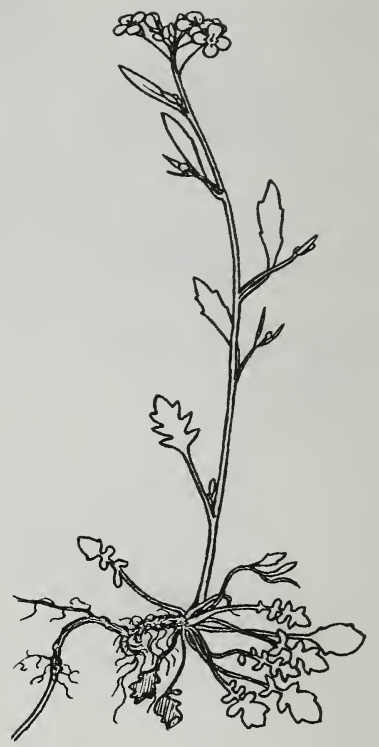

Arnica latifolia

ARLA8

broadleaf arnica

Compositae

HABIT: 4-25 inches.

KEY CHARACTERS: Basal leaves smaller than the stem leaves, basal leaves usually withered by flowering time, stem leaves $3 / 4-4$ inches long and $1 / 5-3$ inches wide, usually toothed, petiole shorter than the blades. Flowers yellow, 1-5 per plant. Fruit achenes.

HABITAT: Open woods, meadows, heath.

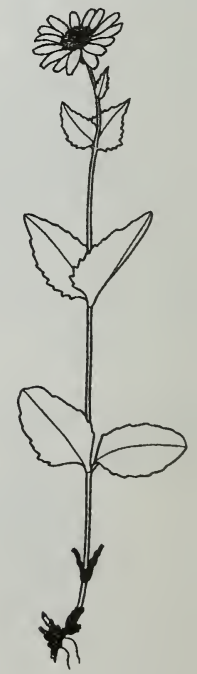


HABIT: Stems from a stout, woody base with short runners.

KEY CHARACTERS: Leaves at base bipinnately dissected into about 5-7 pairs, with linear, pointed, ultimate lobes. Stem leaves are reduced. Nodding dense cluster of flowers, the lower long-stalked. Yellow, fuzzy, disc flowers. Smooth fruit.

HABITAT: Meadows, in the mountains to 6500 feet.

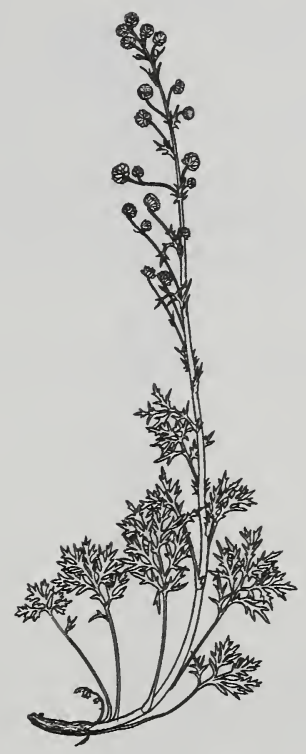

Artemisia tilesii

Aleutian mugwort

HABIT: $1-4$ feet.

KEY CHARACTERS: Leaves alternate, 1-2 times pinnately divided, hairy underneath. Flowers yellow, tinged with red, numerous nodding heads in spike-like inflorescences. Fruit hairless achenes.

HABITAT: Lowlands to mountains. Roadsides, gravelly or sandy areas.

ARTI

Compositae

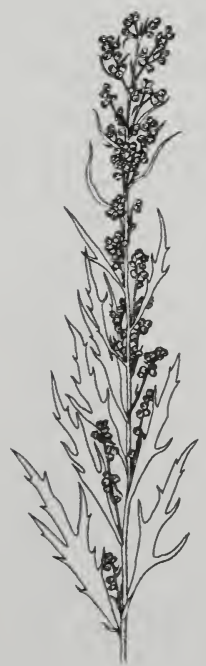


Aruncus sylvester goatsbeard
ARSYA

Rosaceae

HABIT: 3-6 feet.

KEY CHARACTERS: Leaves alternate, ternate, doubly toothed. Flowers white, several in terminal clusters. Fruit upright follicles.

HABITAT: Moist forests, meadows, streambanks.

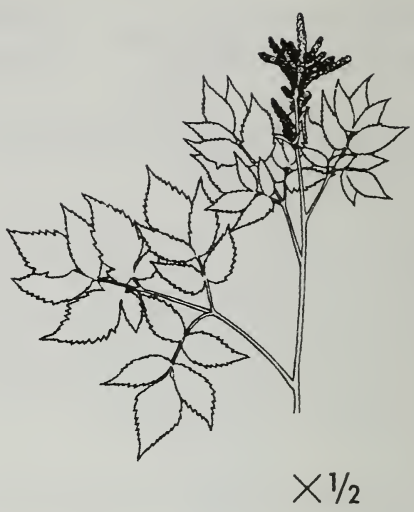

Boschniakia rossica ground-cone

BORO

Orobanchaceae

HABIT: Cone-like, 4-16 inches.

KEY CHARACTERS: Leaves scale-like, yellowish to purplish. Flowers purplish, several in terminal inflorescence.

HABITAT: Forests, beaches. Commonly found under alder stands. Parasitic on alder roots. 


\section{Callitriche hermaphroditica CAHE2}

northern waterstarwort

Callitrichaceae

HABIT: Slender plants, 2-10 inches long.

KEY CHARACTERS: Linear 1-veined leaves with indentation at tip. Fruit with conspicuous wings. Plant dark green.

HABITAT: Submerged in shallow ponds.

Callitriche verna spring waterstarwort
CAVE2

Callitrichaceae

HABIT: Slender plants, 2-10 inches long.

KEY CHARACTERS: Linear 1-veined leaves with indentation at tip. Fruit not distinctly winged. Plant light green.

HABITAT: Floating in shallow ponds, mud.

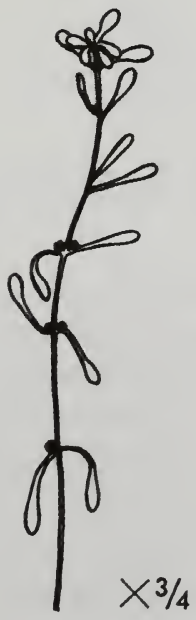


Caltha palustris

yellow marsh-marigold
CAPA5

Ranunculaceae

HABIT: Decumbent, 4-24 inches.

KEY CHARACTERS: Leaves basal and on stem, toothed. Stem hollow. Flowers yellow, often greenish or purplish on back, solitary, terminal, with 5-8 petal-like sepa1s. Fruit follicles, with hooked beak. Poisonous.

HABITAT: Slow running water, moist places.

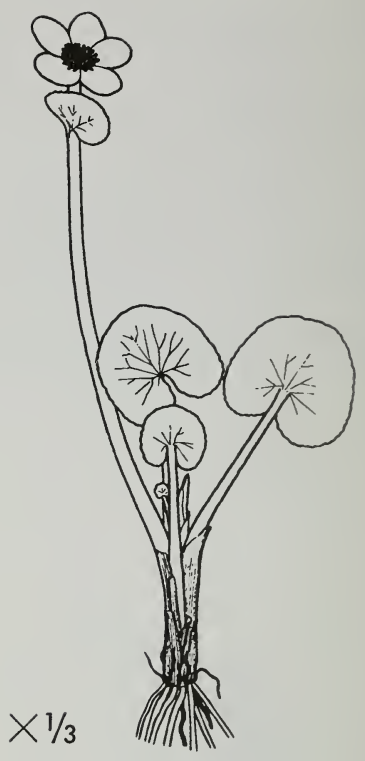

Campanula lasiocarpa mountain harebell
CALA7

Campanulaceae

HABIT: Stems from thin, branching rhizomes.

KEY CHARACTERS: Leaves at base are long-stalked, tear-shaped and pointed, entire or usually coarsely dentate. Stem leaves are not stalked and are gradually reduced. Single, large blue flowers with linear, pointed, narrow-lobed segments.

HABITAT: Alpine heaths, sandy tundra, in

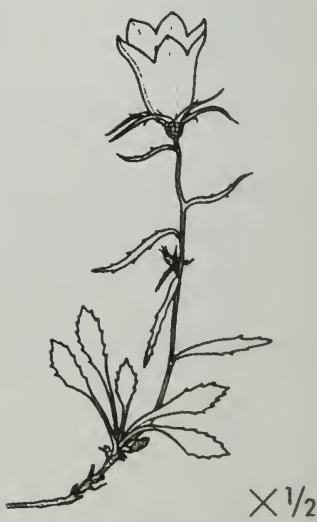
the mountains to at least 5000 feet. 
Campanula rotundifolia bluebells of Scotland
CARO2

Campanulaceae

HABIT: One to several prostrate to upright stems, to 8 inches.

KEY CHARACTERS: Leaves narrow, with few teeth. Flowers purplish-blue, solitary, terminal. Sepals bent backward. Fruit nodding capsules.

HABITAT: Open areas, grassy slopes, rock outcrops along coast.

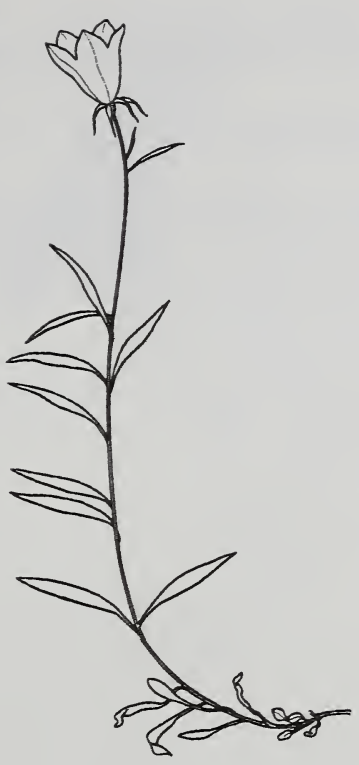

Cardamine umbellata

CAUM3

little western bittercress

Cruciferae

HABIT: To 20 inches.

KEY CHARACTERS: Basal leaves with 1-3 pairs of leaflets and a 3-lobed terminal leaflet. Stem leaves alternate, with narrower leaflets. Flowers small, white, in terminal inflorescence. Fruit long, thin capsules (siliques).

HABITAT: Wet areas, disturbed areas.

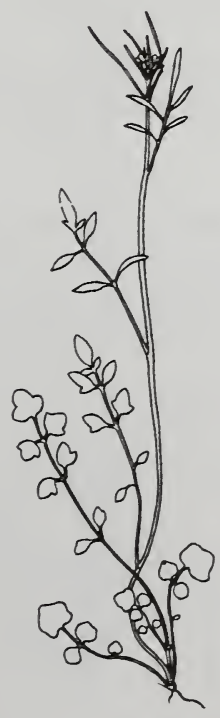


Castilleja unalaschcensis CAUN4

Alaska Indian paintbrush Scrophulariaceae

HABIT: 8-32 inches.

KEY CHARACTERS: Stem leaves pubescent, with 3-5 veins. No basal leaves. Flower bracts yellowish, lobed, at top of stem. Fruit capsules.

HABITAT: Open forests, meadows, tidal flats.

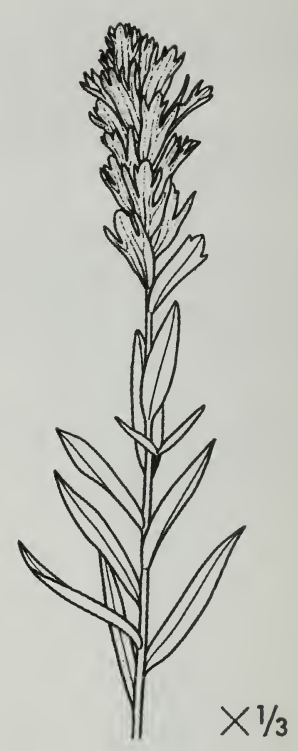

Cicuta douglasii

western water hemlock
CIDO

Umbelliferae

HABIT: Stout with single stems, or a few together, from tuberous and chambered roots.

KEY CHARACTERS: Leaves 1-3 pinnate with serrate, oblong leaflets. Several umbels of flowers with 12-20 rays. Fruit ovate to circular. Poisonous.

HABITAT: Marshes, along streams.

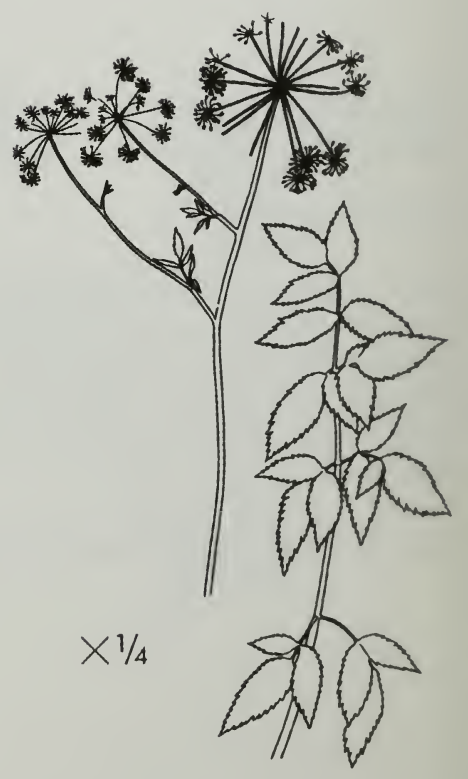


Cicuta mackenzieana

CIMA

Mackenzie's water hemlock Umbelliferae

HABIT: Stout, single stems or a few together, from tuberous roots.

KEY CHARACTERS: Leaves 1-3 pinnate, leaflets lanceolate and thin. Several umbels of flowers with 7-14 rays. Ripe fruit much broader than long. Poisonous.

HABITAT: Marshes.

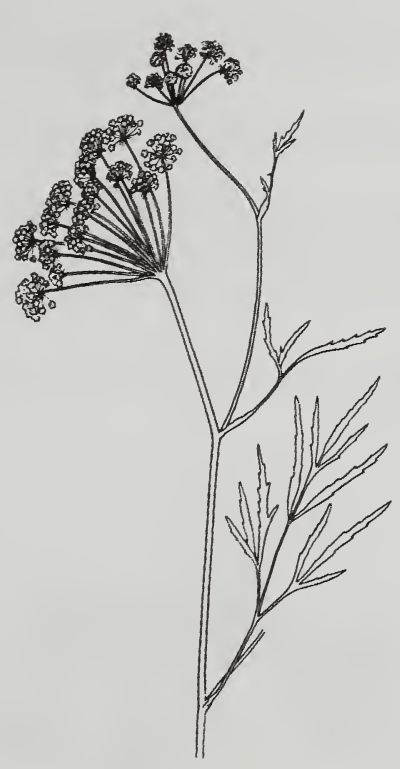

Circaea alpina

CIAL

enchanter's nightshade

Onagraceae

HABIT: 2-10 inches.

KEY CHARACTERS: Leaves opposite, toothed. Flowers white to pink, in racemes, petals 2-lobed. Fruits covered with soft bristles.

HABITAT: Moist forests, streambanks, seeps, with Sitka alder along beaches.

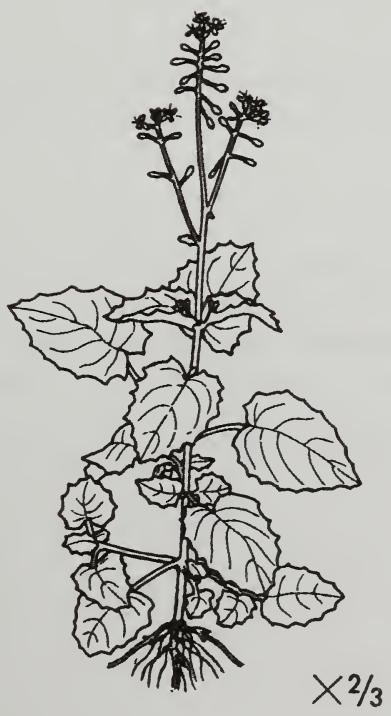


Coeloglossum viride longbract frog orchid
COVI6

Orchidaceae

HABIT: Single upright stem, 21/2-25 inches.

KEY CHARACTERS: Stems with several leaves. Flowers green to yellowish green in several to many-flowered raceme.

HABITAT: Tundra, heath, woods, meadows.

Conioselinum chinense western hemlock-parsley
COCHP

Umbelliferae

HABIT: 6-40 inches.

KEY CHARACTERS: Leaves alternate, 2-3 times pinnately compound, leaflets again lobed, with inflated petiole. Flowers white, in umbels. Fruits with lateral wings.

HABITAT: Meadows, sandy shores.

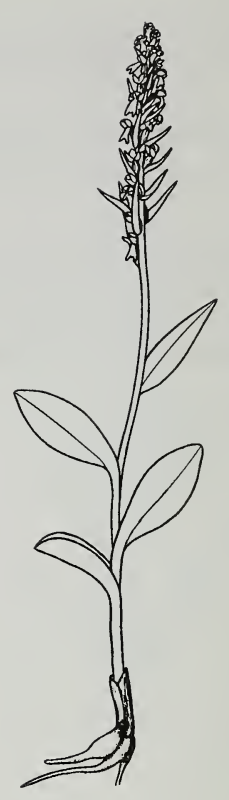


Coptis aspleniifolia

fern leaf goldthread

HABIT: Evergreen, 4-14 inches.

KEY CHARACTERS: Leaves basal, toothed, with 5 leaflets. Flowers white, 1-3 per plant, on long leafless stem. Fruit follicles, speading in wheel-shape.

HABITAT: Forests, muskegs, moist places.

COAS

Ranunculaceae

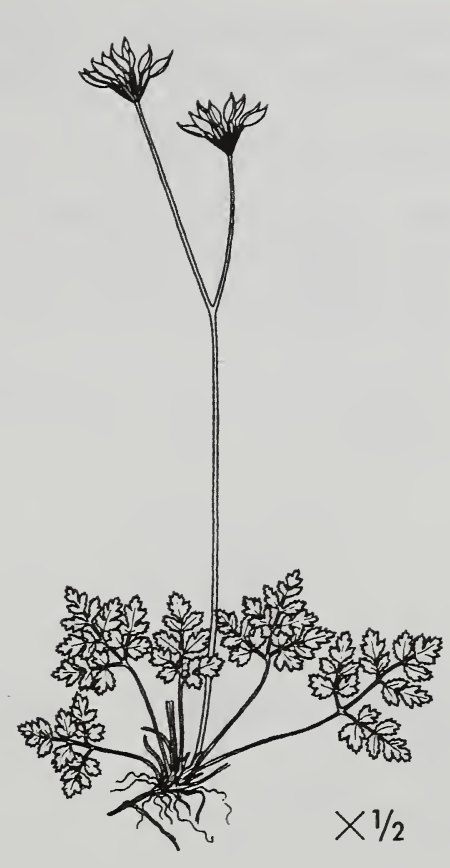

Coptis trifolia trifoliate goldthread

HABIT: Evergreen, 2-6 inches.

KEY CHARACTERS: Leaves pale below, toothed, shiny, with 3 leaflets. Flowers white, solitary, on long leafless stem. Fruit follicles.

HABITAT: Forests, meadows, muskegs, tundra.
COTR2

Ranunculaceae

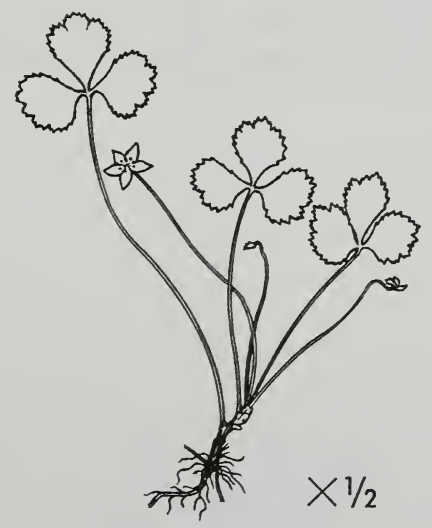


Cornus canadensis

bunchberry
COCA13

Cornaceae

HABIT: 2-8 inches.

KEY CHARACTERS: Leaves 4-6 in a terminal whorl with 1-2 pairs much smaller, opposite stem leaves below. Flower bracts white, petal-like. Fruit clustered red berries.

HABITAT: Spruce and birch forests, muskegs.

DEGL3

Delphinium glaucum larkspur

Ranunculaceae

HABIT: $1 \frac{1}{2}-5$ feet.

KEY CHARACTERS: Leaves alternate, palmately 5-7 lobed, the lobes divided and toothed. Flowers dark violet-purple, spurred, in many-flowered terminal raceme. Fruit hairless follicles.

HABITAT: Wet meadows, streambanks.
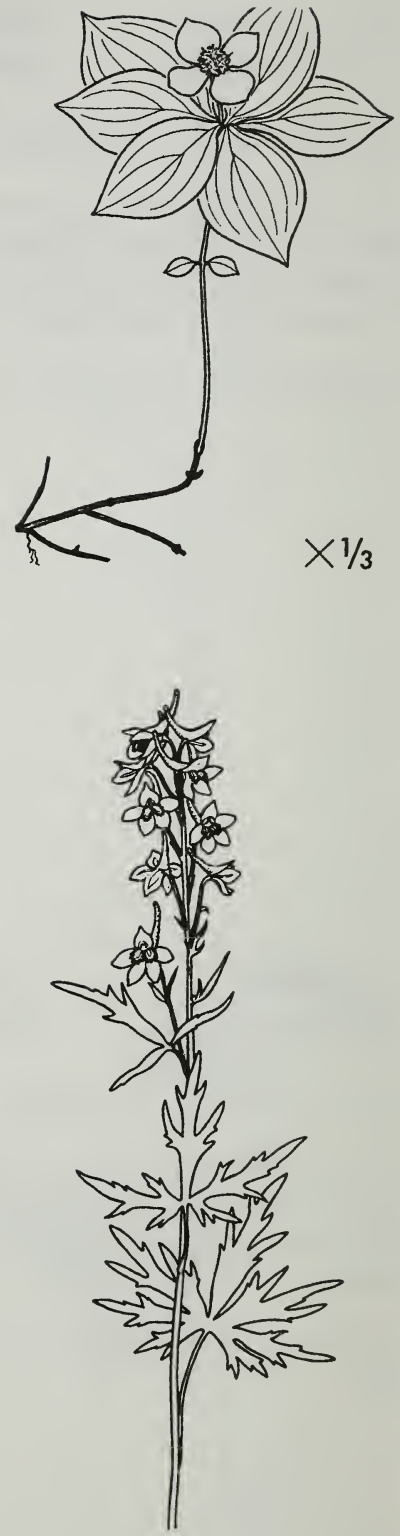
Dodecatheon pulchellum DOPU

pretty shooting star

Primulaceae

HABIT: 5-24 inches.

KEY CHARACTERS: Leaves basal, thick, with long, winged petiole. Flowers purplish-lavender with a yellow ring at base, several per long leafless stem. Fruit ovoid capsules.

HABITAT: Muskegs, open areas, saline meadows.

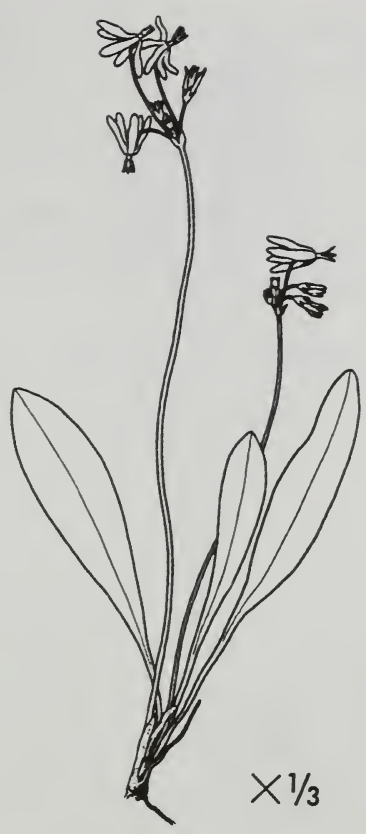

Drosera rotundifolia

DRRO

round-leaf sundew

Droseraceae

HABIT: 3 inches.

KEY CHARACTERS: Leaves in basal rosettes, nearly round, lying flat on the ground, with sticky red-stalked glands. Flowers white, several in leafless longstemmed inflorescence. Fruit capsules.

HABITAT: Muskegs, swamps, wet areas.

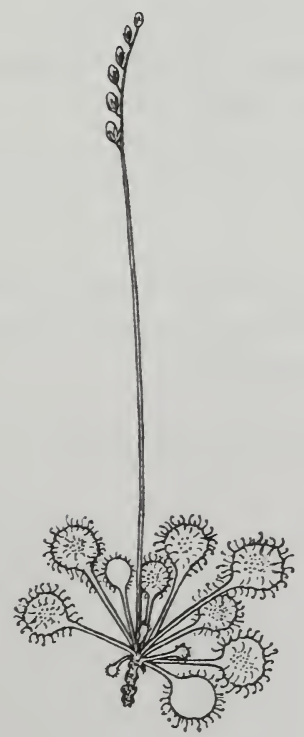


Epilobium adenocaulon

northern willow herb
EPAD

Onagraceae

HABIT: Upright, branched. 4-30 inches

KEY CHARACTERS: Leaves mostly opposite, toothed. Flowers small, white to red. Fruit capsules.

HABITAT: Disturbed sites, roadsides.

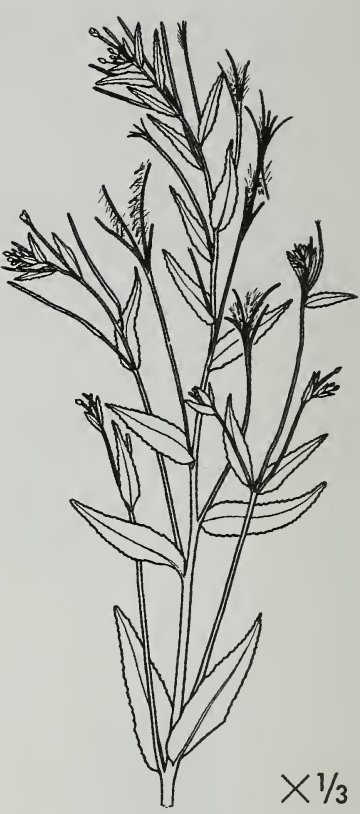

Epilobium anagalladifolium EPAN4 alpine willow herb Onagraceae

HABIT: Low-growing, single stem, 4-6 inches.

KEY CHARACTERS: Leaves opposite, small. Flowers small, reddish-violet to pink. Fruit capsules.

HABITAT: Moist places.

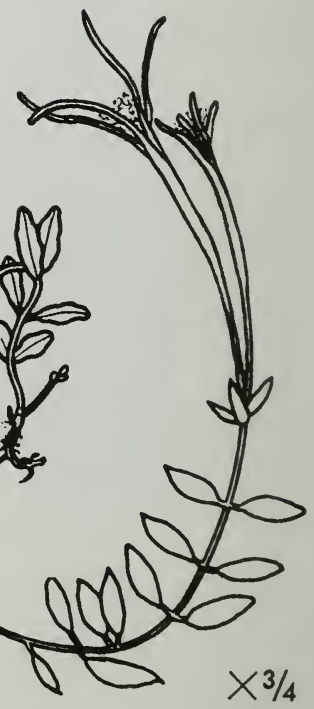


Epilobium angustifolium tall fireweed
EPAN2

Onagraceae

HABIT: Unbranched, $1 \frac{1}{2}-5$ feet.

KEY CHARACTERS: Leaves alternate, pale and distinctly veined underneath. Stem densely leafy. Flowers large, lilac-purple, in terminal raceme. Fruit hairy capsules, 1-4 inches long.

HABITAT: Forests, meadows, gravel bars, beach fringe, burned areas.

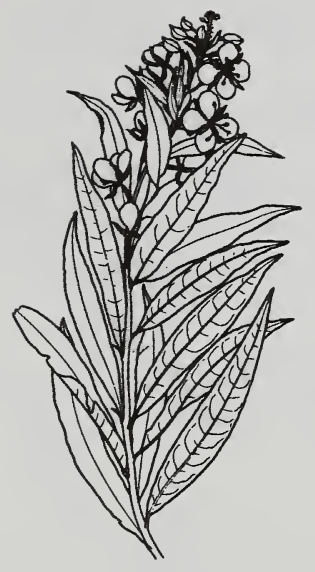

Epilobium glandulosum fringed willow herb

EPGL4

Onagraceae

HABIT: $10-40$ inches.

KEY CHARACTERS: Stems single or branched in upper portion of plant. Leaves mostly opposite, serrate. Petals pink to purplish.

HABITAT: Moist sites in woods, thickets, meadows, and beaches.

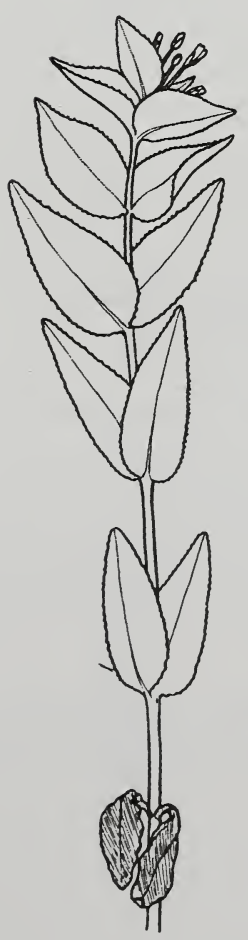


Epilobium hornemannii EPHO

Hornemann's willow herb Onagraceae

HABIT: $1 \frac{1}{2-10}$ inches.

KEY CHARACTERS: Stems mostly single. Leaves mostly opposite (the upper ones may be alternate), sparsely serrate. Petals lilac-pink.

HABITAT: Near seeps and springs, along streams, in moist meadows, bogs.

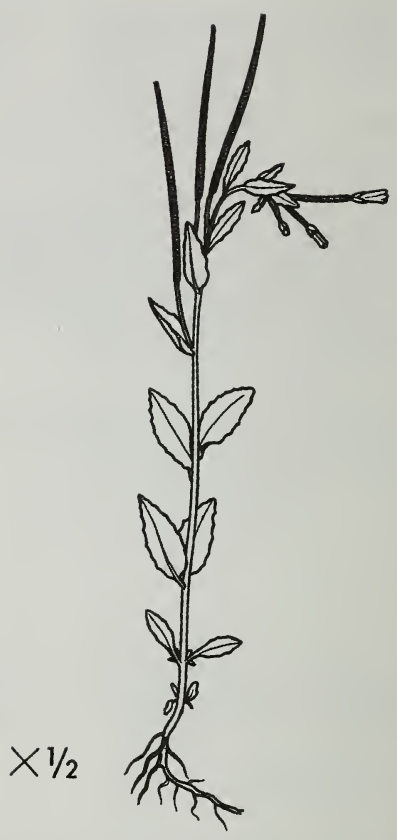

Epilobium latifolium dwarf fireweed

EPLA

Onagraceae

HABIT: Decumbent, 4-12 inches.

KEY CHARACTERS: Leaves alternate, whitish, finely pubescent, fleshy, not veiny. Flowers large, purple or rose, numerous from leaf bases. Fruit purplish hairy capsules.

HABITAT: To 6000 feet. Meadows, steambanks, river bars, scree slopes.

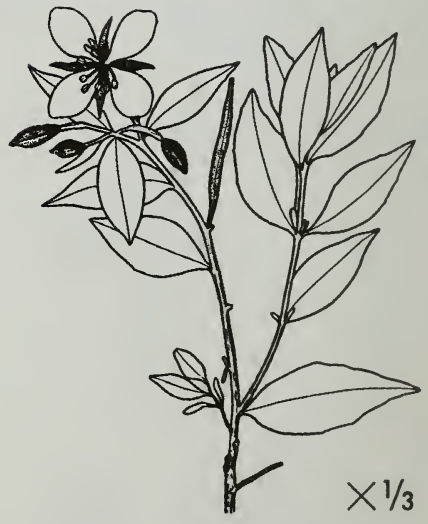


Epilobium leptocarpum EPLE slenderfruit willow herb Onagraceae

HABIT: Many branched, usually less than 4 inches.

KEY CHARACTERS: Leaves mostly opposite, few teeth. Petals whitish to pink.

HABITAT: Moist places.

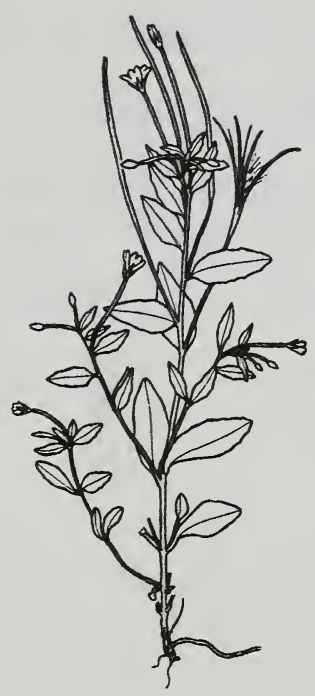

EPPA

Onagraceae
Epilobium palustre swamp willow herb

HABIT: Branched or unbranched, 4-16 inches.

KEY CHARACTERS: Leaves opposite low on stem, alternate high on stem, narrow, with no petiole. Flowers small, pink or whitish. Fruit hairy capsules.

HABITAT: Meadows, muskegs, steambanks, wet places.

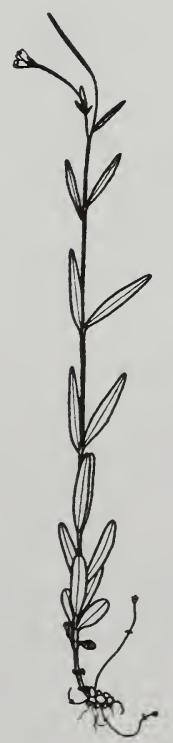


Erigeron peregrinus subalpine fleabane
ERPE3

Compositae

HABIT: 2-24 inches.

KEY CHARACTERS: Leaves alternate, with hairy edges and no petiole. Flowers white, pink, purple, or blue, usually 1 per plant and solitary, terminal. Fruit achenes.

HABITAT: Open forests, meadows, muskegs, alpine.

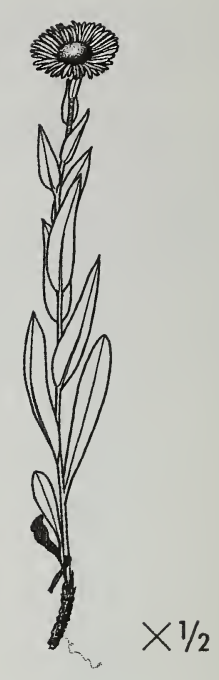

Fauria crista-galli

FACR

deer cabbage

Gentianaceae

HABIT: 8-12 inches.

KEY CHARACTERS: Leaves basal, kidney-shaped, fleshy, with rounded teeth and slightly notched tip. Flowers white, in leafless long-stemmed inflorescence. Fruit capsules.

HABITAT: Open forests, muskegs, swamps, wet meadows, seeps.

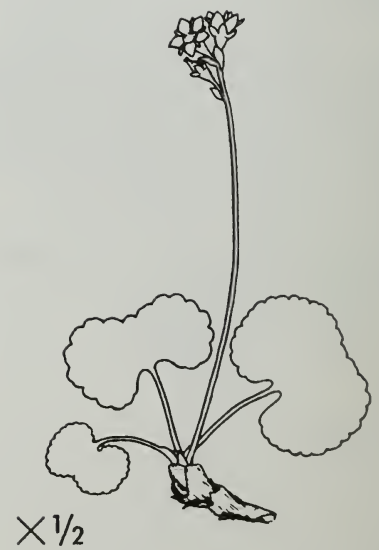


Fragaria chiloensis beach strawberry
FRCH

Rosaceae

HABIT: Plant with stout rhizomes and long, brown stolons.

KEY CHARACTERS: Thick leaves with three leaflets, coarsely serrate, the upper surface green and shiny, the lower surface pale and usually hairy. Petals white. Fruit mostly at least $1 / 2$ inches in diameter.

HABITAT: Along the coast in woods, meadows, and gravelly beaches.

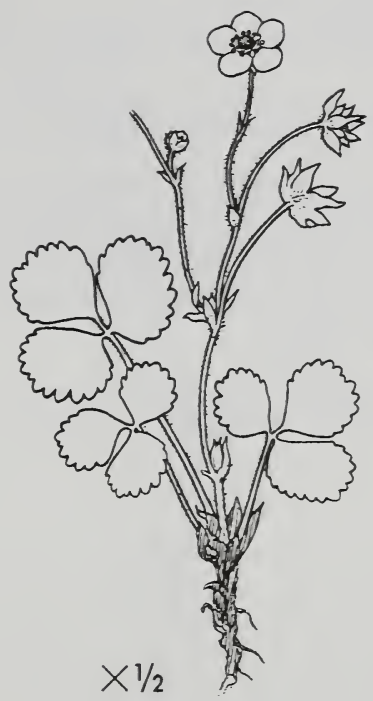

Fritillaria camschatcensis chocolate lily

FRCA5 Liliaceae

HABIT: 8-24 inches.

KEY CHARACTERS: Leaves 5-9 in a whorl. Flowers purple-black, 1-several per stem, terminal, nodding, with unpleasant odor. Fruit capsules.

HABITAT: Meadows, tide flats.

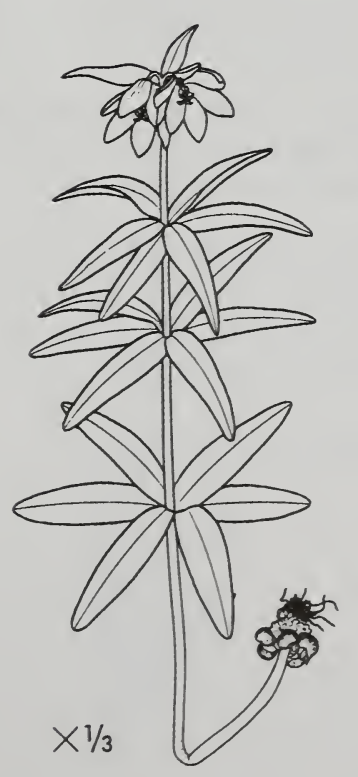


Galium boreale northern bedstraw
GABO2

Rubiaceae

HABIT: Upright, branched, 8-32 inches.

KEY CHARACTERS: Leaves 4 in a whorl, rounded at the tip, with 3 veins and no petiole. Stems square. Flowers white to cream, several in terminal inflorescences. Fruit with hairs.

HABITAT: Meadows, rocky slopes.

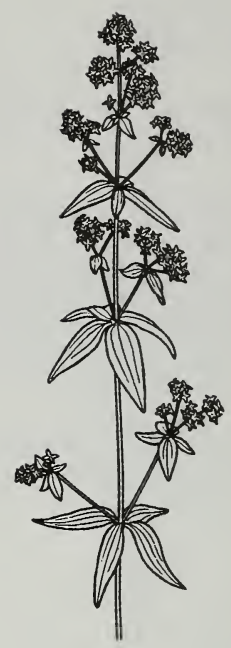

Galium trifidum

threepetal bedstraw
GATR2

Rubiaceae

HABIT: Weak, slender, branched stems with upper internodes rough to the touch, from slender rhizome.

KEY CHARACTERS: Leaves in whorls of 4 , linear to oblong, blunt and bent downward. Whitish, lobed flowers, single or 3 together. Smooth fruit.

HABITAT: Wet places.

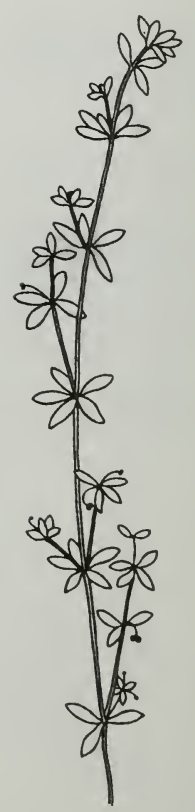


Galium triflorum fragrant bedstraw
GATR3

Rubiaceae

HABIT: Perennial, sprawling, $8-40$ inches long.

KEY CHARACTERS: Leaves 6 in a whorl, pointed at tip, with 1 vein and no petiole. Stems square and bristly. Flowers greenish-white, several per plant, usually 3 per stalk, from leaf bases. Fruits with hairs and prickles.

HABITAT: Moist forests, thickets, open areas.

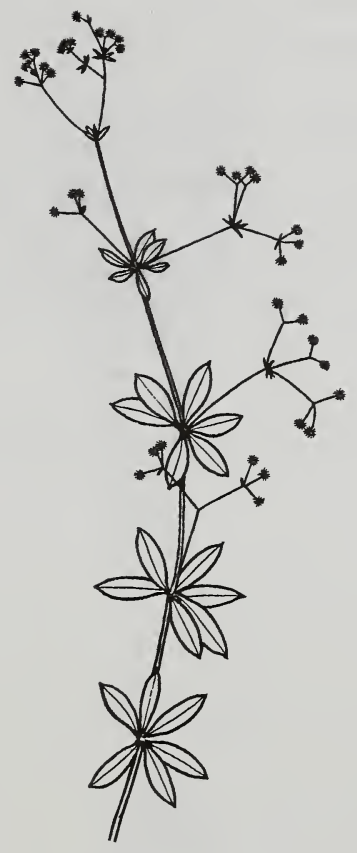

Gentiana douglasiana

GEDO swamp gentian

Gentianaceae

HABIT: Branched, 2-11 inches.

KEY CHARACTERS: Leaves opposite. Flowers white, bluish on outside, solitary, or in inflorescence, terminal or from leaf bases. Fruit oblong stalkless capsules.

HABITAT: Muskegs, wet meadows, alpine.

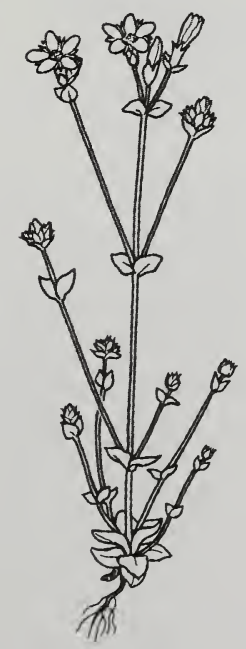


Gentiana glauca pale gentian
GEGL

Gentianaceae

\section{HABIT: 1-6 inches}

KEY CHARACTERS: Smooth, yellowish-green leaves in clusters. Stem leaves in 1-3 pairs, elliptical to rounded. Inner flower blue, dark blue, or greenish-blue with ovate, obtuse lobes.

HABITAT: Meadows in the alpine and subalpine zones, to at least 5000 feet.

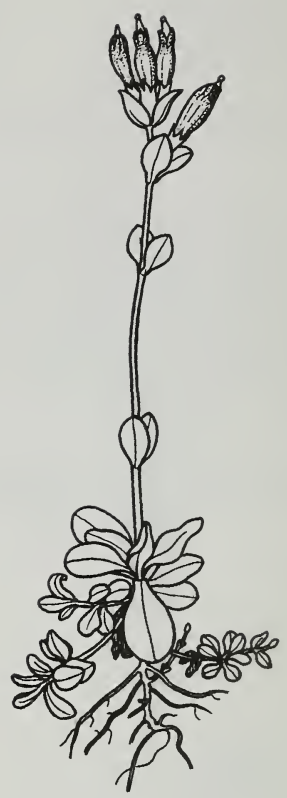

Gentiana platypetala spotted gentian

HABIT: 4-14 inches.

KEY CHARACTERS: Leaves opposite, with no petiole. No basal leaves. Flowers blue, mostly solitary, terminal, with no stalk. Fruit oblong capsules.

HABITAT: Sea level to alpine. Wet meadows, grassy slopes.

\section{GEPL}

Gentianaceae

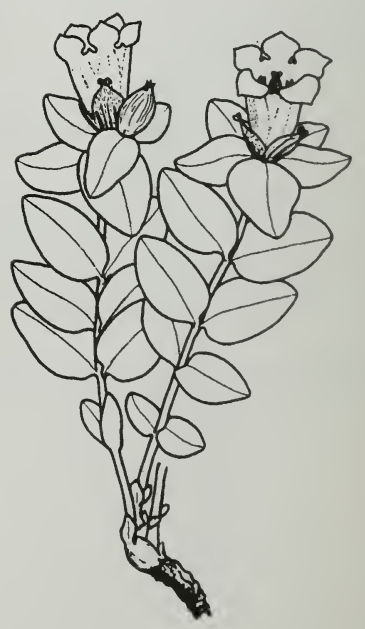


Geocaulon lividum

northern comandra
GELI2

Santalaceae

HABIT: To 6 inches.

KEY CHARACTERS: Leaves alternate, often bi-colored. No basal leaves. Flowers inconspicuous, from leaf bases, with no petals. Fleshy orange fruits.

HABITAT: Poplar flats, muskeg, dry places, tundra.

Geranium erianthum

GEER2

northern geranium

Geraniaceae

HABIT: 8-32 inches.

KEY CHARACTERS: Basal leaves round in outline, palmately 3-5 lobed, pubescent, with long petiole. Stem leaves with no petiole. Flowers rose or violet, 3-5 in clusters. Fruit pubescent.

HABITAT: Forests, meadows, streambanks, rocky areas, alpine, beaches.
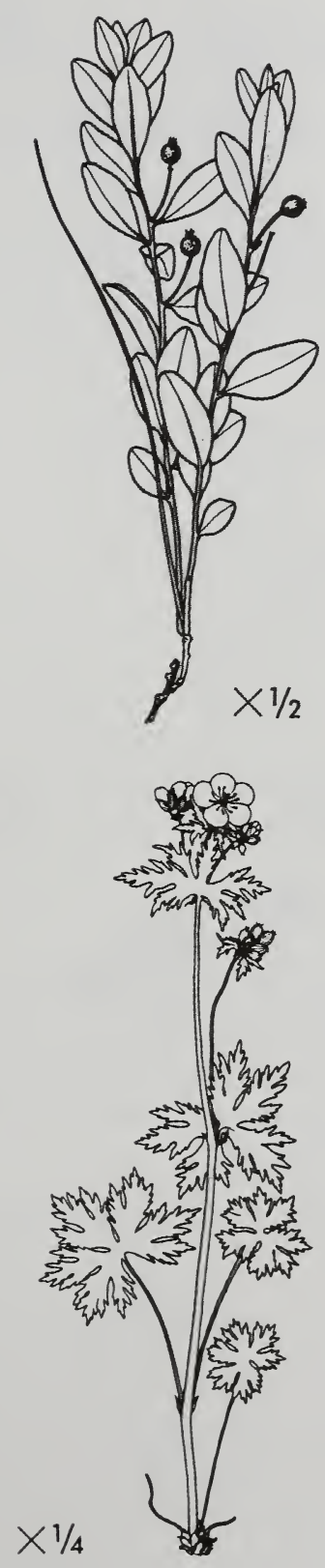
Geum calthifolium

calthaleaf avens
GECA6

Rosaceae

HABIT: Stems from dark, stout woody base.

KEY CHARACTERS: Leaves at base fuzzy on both sides, with short, yellow hairs. Very small lower lobes and a large, orbicular end lobe. Yellow, heart-shaped flower petals.

HABITAT: Wet meadows.

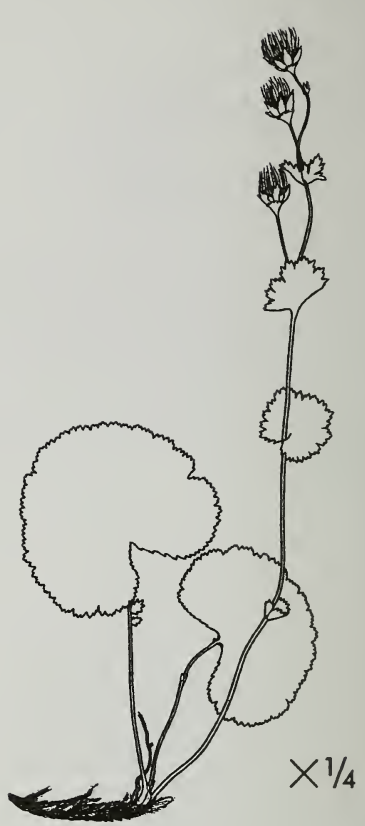

Geum macrophyllum large-leaf avens
GEMA4

Rosaceae

HABIT: $11-40$ inches.

KEY CHARACTERS: Basal leaves lyre-shaped and pinnately compound, hairy. Stem leaves with fewer leaflets. Stems hairy. Flowers yellow, several in inflorescences. Fruit hairy nutlets in burr-like heads.

HABITAT: Forests, muskegs, beaches.

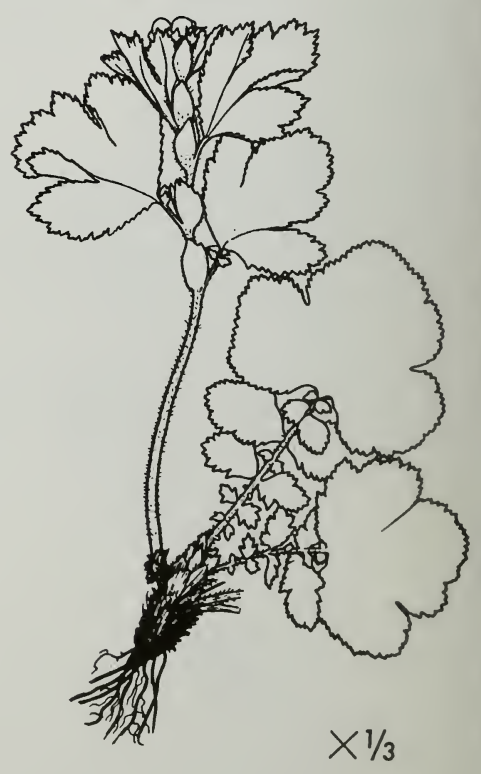


Hedysarum alpinum

alpine sweetvetch
HEAL

Leguminosae

HABIT: Upright to decumbent stems, 4-40 inches tall.

KEY CHARACTERS: Compound leaves with 9-23 leaflets. Flowers in raceme from 1-7 inches long. Petals pink to pink purple (rarely white). Fruits are loments with 1-4 segments.

HABITAT: Alpine tundra, heath, woods. Mostly on sandy or gravelly soils.

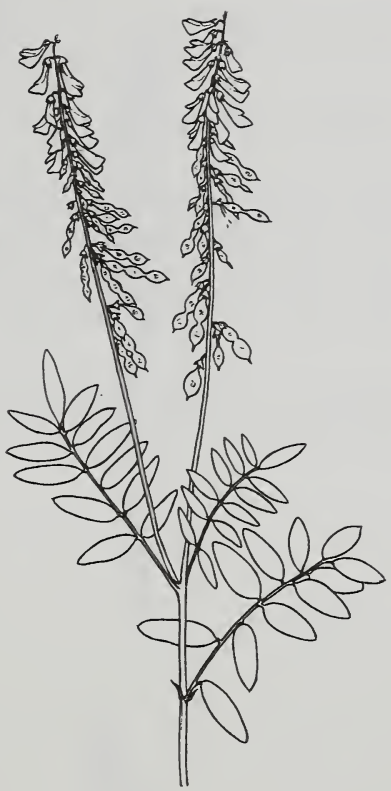

Heracleum lanatum

HELA4 cow parsnip

Umbelliferae

HABIT: $1 \frac{1}{2}-6$ feet.

KEY CHARACTERS: Leaves alternate, large, lobed and toothed, upper with inflated sheaths. Flowers white, in umbels. Fruit with lateral wings.

HABITAT: Forests, meadows, streambanks, beaches.

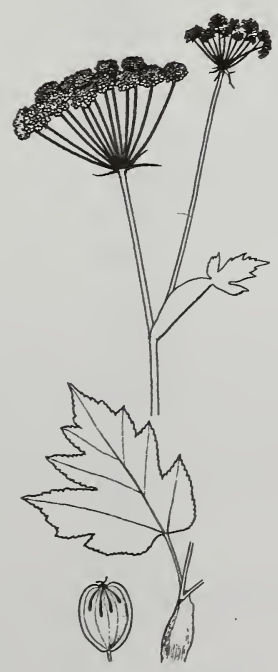


Heuchera glabra alpine heuchera
HEGL5

Saxifragaceae

HABIT: 6-24 inches.

KEY CHARACTERS: Leaves mostly basal, 3-5 lobed, toothed. Flowers white, in long-stemmed inflorescence with 1-3 reduced leaves. Fruit capsules.

HABITAT: Streambanks, moist rocks, sea cliffs, alpine meadows along seeps.

Hieracium triste

woolly hawkweed
HITR2

Compositae

HABIT: 1-10 inches.

KEY CHARACTERS: Leaves at base tearto spoon-shaped, entire with hairs in margin, long-stalked, smooth above, sparsely fuzzy beneath. Large terminal flower heads, with grayish, long, soft hairs. Short, yellow ligules.

HABITAT: Stony slopes.

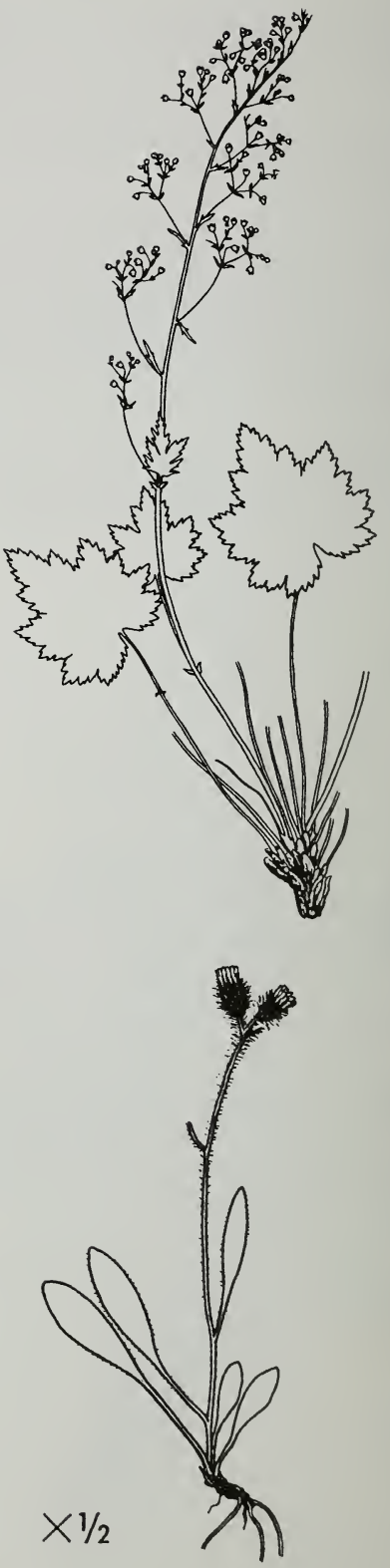


Hippuris tetraphylla

fourleaf marestail
HITE

Haloragaceae

HABIT: Stem from stout, creeping rhizome.

KEY CHARACTERS: Leaves 4-6 in a whorl, $1 / 10-1 / 5$ inches wide, entire, not pointed, and shorter than the internodes.

HABITAT: Shallow ponds and mud flats.

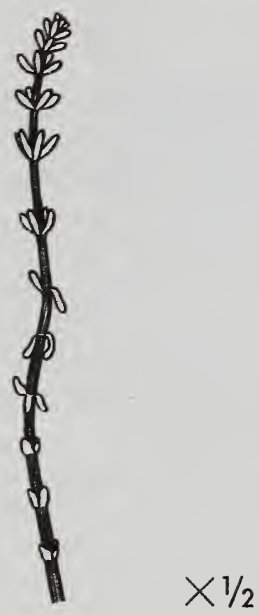

Hippuris vulgaris common marestail
HIVU2

Haloragaceae

HABIT: Stem from stout, creeping rhizome

KEY CHARACTERS: Leaves 6-12 in a whorl, linear, entire, pointed and longer than the internodes. In submerged forms thin, pale green. Small flowers in leaf-notch of submerged leaves.

HABITAT: Shallow ponds, streams, and mud flats.

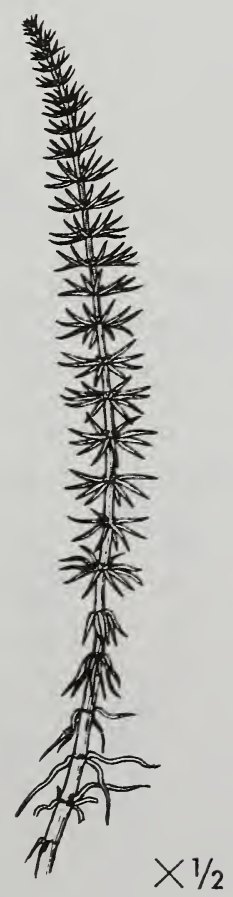


Honckenya peploides seaside sandplant
HOPE

Caryophyllaceae

HABIT: Forming loose to dense mats 4-30 inches broad or more, from deep taproot and horizontal stolons/rhizomes.

KEY CHARACTERS: Leaves opposite, fleshy, lower leaves smaller than those of the middle stem, stem leaves 3-10 pairs or more. Flowers solitary in the upper leaf axils, petals white or greenish-white.

HABITAT: Maritime beaches.

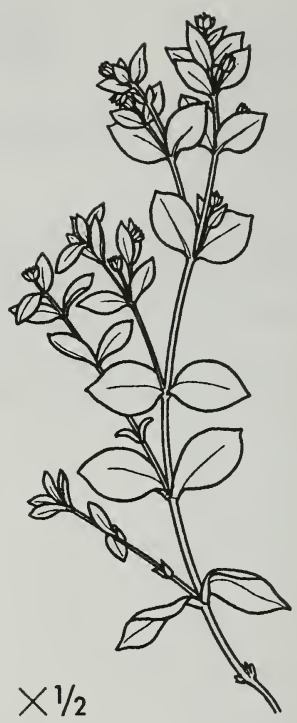

Iris setosa

IRSE

wild iris

Iridaceae

HABIT: 12-28 inches.

KEY CHARACTERS: Leaves sword-shaped. Flowers large, blue with dark viens, few per plant. Fruit capsules. Poisonous.

HABITAT: Meadows, shores, tidal flats.

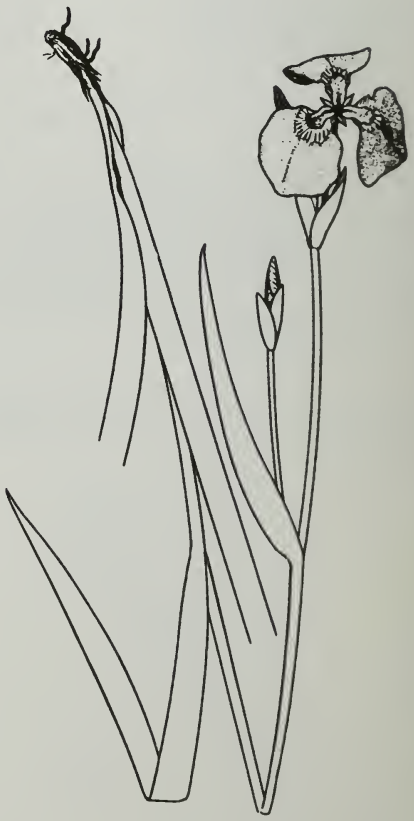


Lathyrus maritimus beach pea
LAMA3

Leguminosae

HABIT: From rhizomes.

KEY CHARACTERS: Fuzzy. Leaves with 6-12 leaflets, tendrils simple to branched. Stipules leaf-like. Pink-purple flowers.

HABITAT: In coastal areas on beaches, strands, and cliff bases.

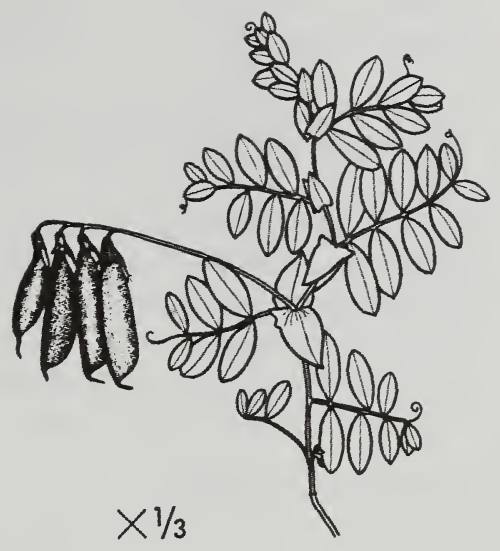

Lathyrus palustris vetchling
LAPA4

Leguminosae

HABIT: From slender rhizomes.

KEY CHARACTERS: Fuzzy. Leaves with 4-8 linear leaflets, branched tendrils. Stipules much smaller than the leaflets. Winged stem. Bluish-violet flowers.

HABITAT: Meadows, tidal flats, beaches, woods, lake shores.

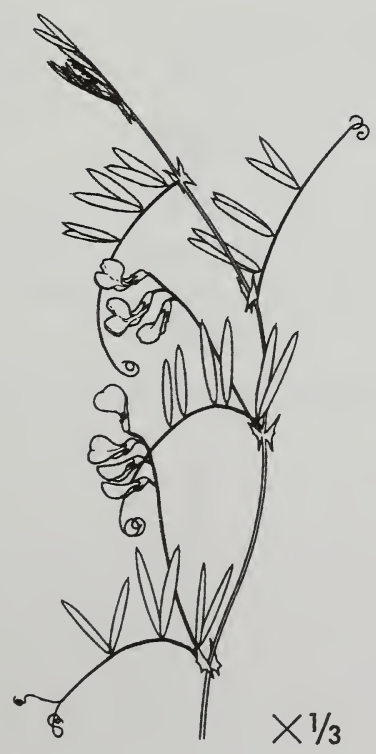


Ligusticum scoticum beach lovage
LISC3

Umbelliferae

HABIT: Single stemmed, smooth and reddish-violet at the base, from a thick root.

KEY CHARACTERS: Thick leaves, divided into threes with ovate, coarsely-toothed leaflets. Stem leaves reduced, with long, often violet, sheaths. Flowers in rays of 7-11 umbels, with white or pinkish petals. Fruit has 3 winged ribs on back.

HABITAT: Seashores.

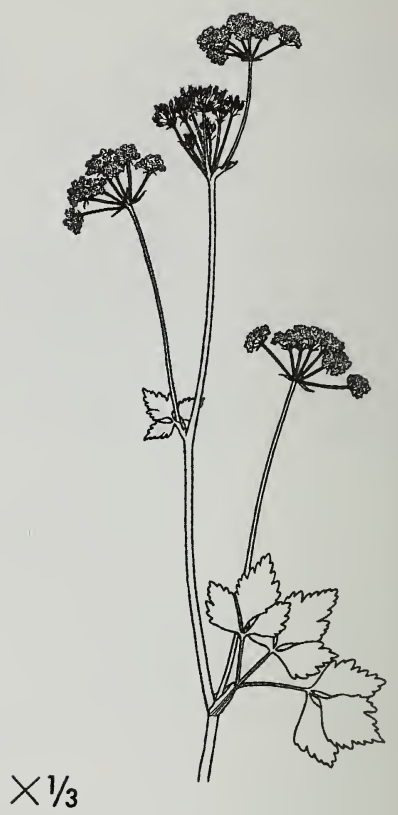

Listera borealis

northern twayblade

HABIT: To 8 inches.

KEY CHARACTERS: Leaves paired on stem. Flowers green or yellowish-green, in terminal raceme. Lowest petal with 2 broad lobes. Fruit capsules.

HABITAT: Moist forests, muskegs.

\section{LIBO4}

Orchidaceae

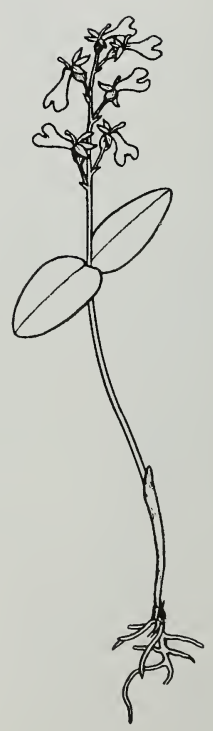


HABIT: 4-14 inches.

KEY CHARACTERS: Leaves paired on stem. Flowers yellowish-green, in terminal raceme. Lowest petal rounded at tip. Fruit capsules.

HABITAT: Evergreen forests.

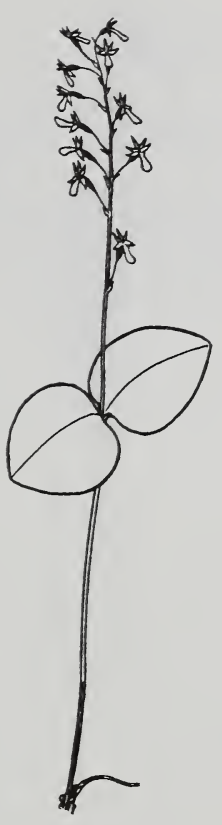

Listera cordata

heart-leaf twayblade
LICO6

Orchidaceae

HABIT: $3-10$ inches.

KEY CHARACTERS: Leaves paired on stem, heart-shaped at base. Flowers green or dark purple, in terminal raceme. Lowest petal with 2 linear lobes. Fruit capsules.

HABITAT: Forests.

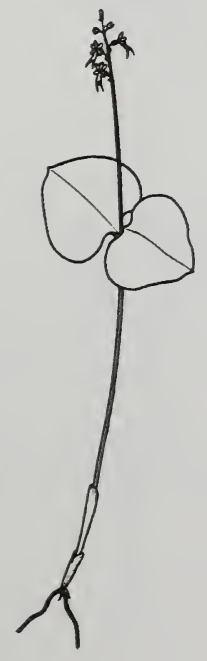


Lloydia serotina common alplily
LLSE

Liliaceae

HABIT: Upright, 2-6 inches.

KEY CHARACTERS: Basal leaves linear, $3 / 4-3$ inches long. Stem leaves alternate, reduced upward, linear, $2 / 5-1 \frac{1}{2}$ inches long. Flowers creamy white.

HABITAT: Alpine tundra and heath.

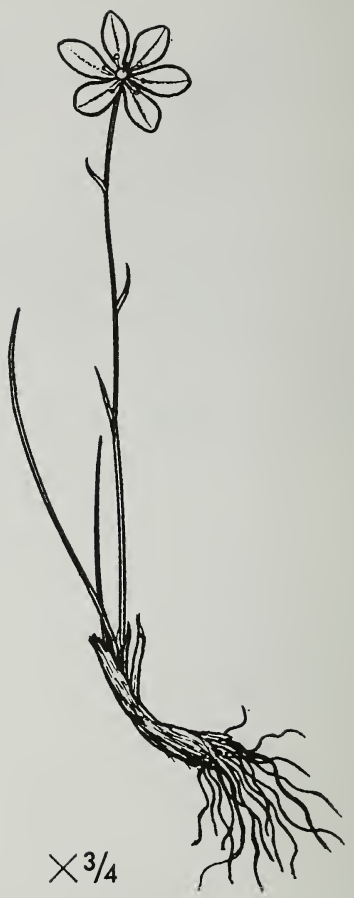

Lupinus nootkatensis

Nootka lupine

HABIT: $1-4$ feet.

KEY CHARACTERS: Leaves alternate, white to brown pubescent on both sides or hairless above, with 5-9 palmately compound leaflets. Flowers blue, in densely pubescent terminal raceme. Fruit peapod-like, black. Poisonous.

HABITAT: Sea level to alpine. Open forests, gravel bars, dry slopes, beaches.
LUNO

Leguminosae

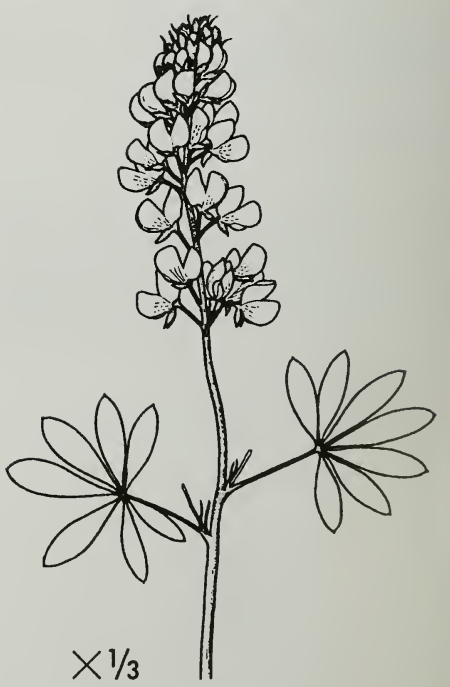


Lysichiton americanum

skunk cabbage
LYAM3

Araceae

HABIT: 1-5 feet.

KEY CHARACTERS: Leaves in basal cluster, 12-51 inches long, fleshy. Flowers small, green, in terminal spike, surrounded by yellow bract. Fruit green to red berries. With unpleasant odor.

HABITAT: Wet forests, edges of muskegs, marshes, streambanks.

Lysimachia thyrsiflora

LYTH2

tufted loosestrife

Primulaceae

HABIT: Upright, 8-30 inches.

KEY CHARACTERS: Lower leaves scale-like. Middle and upper leaves opposite, 1-6 inches long, $1 / 5-2 \frac{1}{2}$ inches wide, dotted with black glands. Flowers on long peduncles in dense racemes from the leaf axils.

HABITAT: Along streams, lake shores, and ponds.
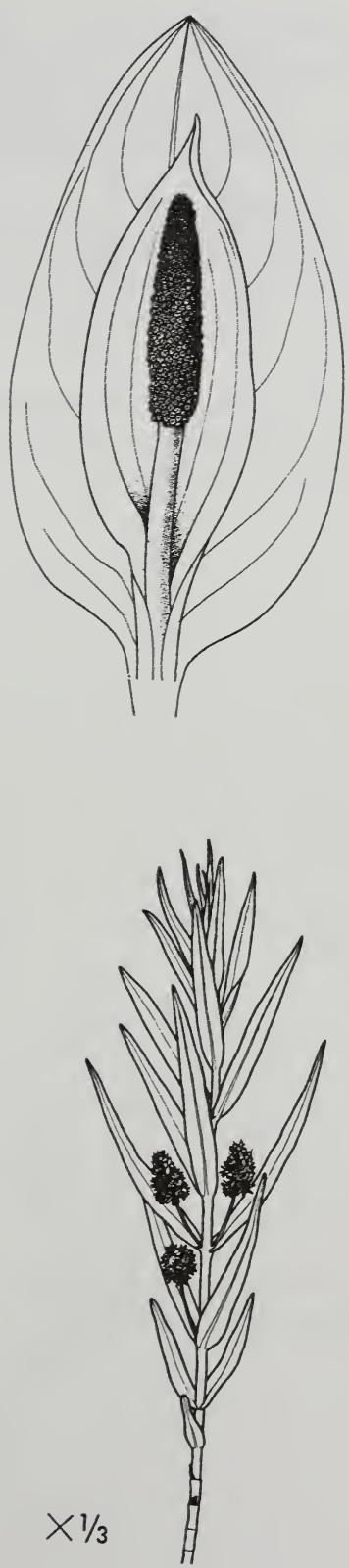
Maianthemum dilatatum deerberry
MADI

Liliaceae

HABIT: Evergreen, 8-23 inches.

KEY CHARACTERS: 2-3 alternate stem leaves, heart- to arrowhead-shaped, parallel veined. Flowers cream to white, in terminal raceme. Fruit red and white mottled berries.

HABITAT: Moist forests, meadows, alder thickets, streambanks, lakeshores, open grassy beach forests.

Menyanthes trifoliata buckbean

\section{METR3}

Gentianaceae

HABIT: Aquatic submerged, to 10 inches.

KEY CHARACTERS: Leaves with 3

leaflets. Flowers white to pink, in terminal raceme, petals with long white hairs. Fruit capsules.

HABITAT: Muskegs, swamps, ponds, wet meadows.
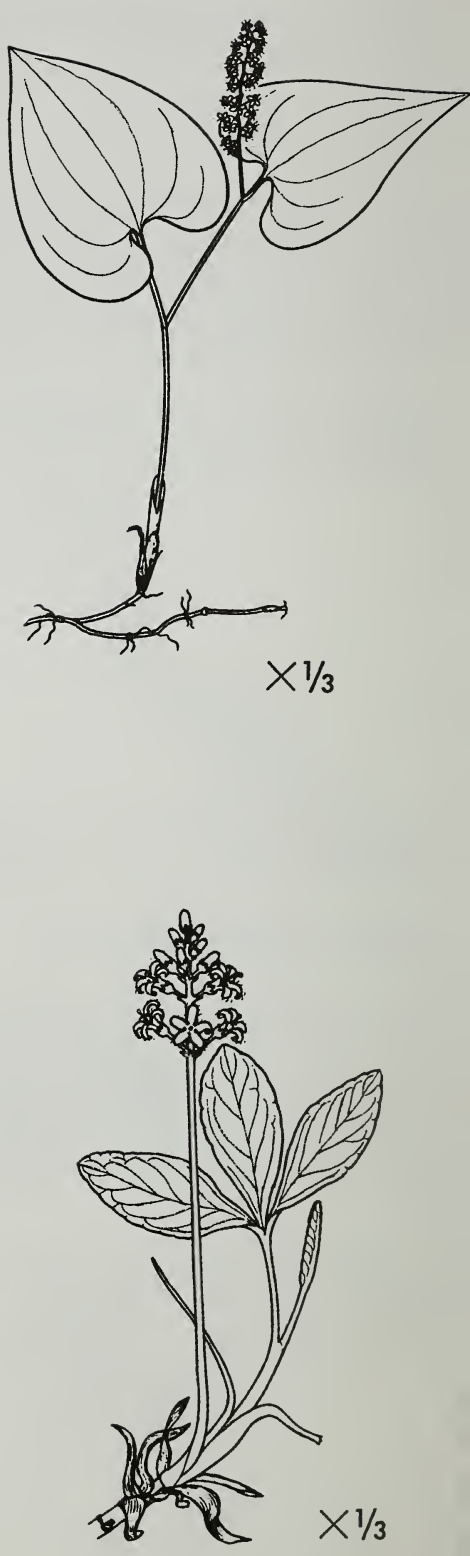
Mertensia paniculata chiming bells
MEPA

Boraginaceae

HABIT: 18-30 inches.

KEY CHARACTERS: Basal leaves with long, winged petiole. Stem leaves alternate, with minute petiole. Flowers blue, in leafless long-stemmed racemes, nodding. Fruit nutlets.

HABITAT: Forests, streambanks, subalpine meadow, gravel bars.

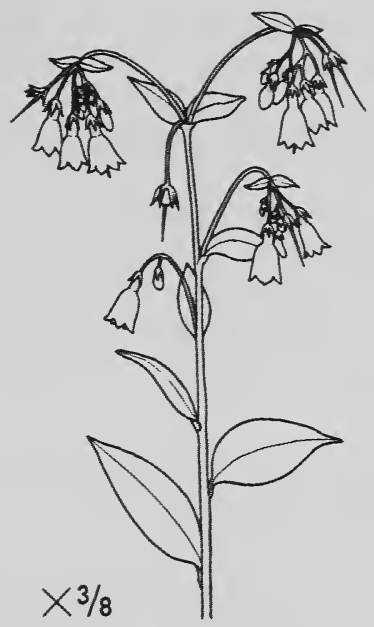

Minuartia arctica MIAR3 arctic stitchwort Caryophyllaceae

HABIT: Upright, branched, to 4 inches.

KEY CHARACTERS: Leaves opposite, hairy underneath and on edges. Largest leaves at middle of stem. Basal leaves narrowly linear. Stem hairy. Flowers white, upright, solitary or 2-5 in terminal inflorescence. Fruit egg-shaped capsules.

HABITAT: Forests, dry meadows, thickets, gravel bars, beaches.

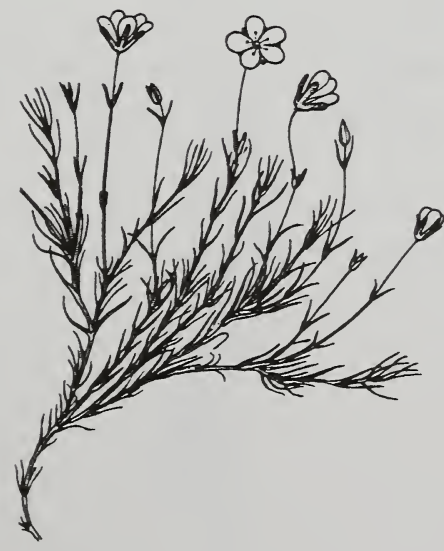


Moehringia lateriflora grove sandwort
MOLA6

Caryophyllaceae

HABIT: Upright, branched, to 4 inches.

KEY CHARACTERS: Leaves opposite, hairy underneath and on edges. Largest leaves at middle of stem. Stem hairy. Flowers white, upright, solitary or 2-5 in terminal inflorescence. Fruit egg-shaped capsules.

HABITAT: Forests, dry meadows, thickets, gravel bars, beaches.

MOUN2

Pyrolaceae

single delight

HABIT: 2-6 inches.

KEY CHARACTERS: Leaves rounded, toothed. Flowers white, waxy, fragrant, nodding, 1 per plant, on long 1-2-bracted stalk. Fruit upright capsules.

HABITAT: Dense forests on rotten wood, organic soil at low elevation.
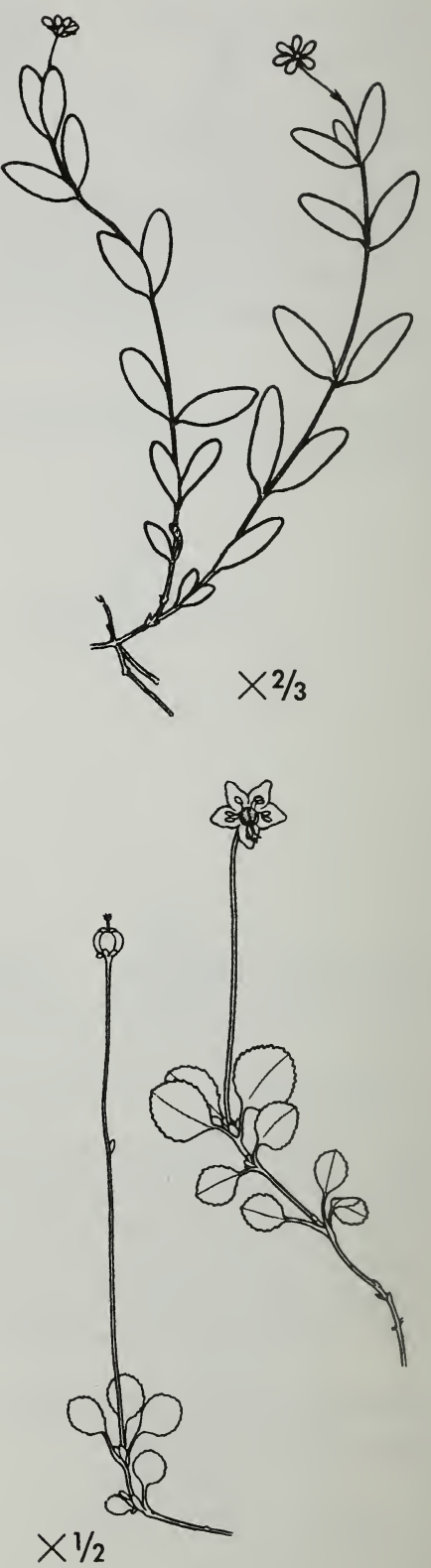
Myosotis alpestris

forget-me-not
MYAL

Boraginaceae

The Alaska State Flower

HABIT: To 20 inches.

KEY CHARACTERS: Basal leaves pubescent, with long petiole. Stem leaves alternate, pubescent, with no petioles. Flowers blue with yellow center, in terminal inflorescence, upright. Fruit nutlets.

HABITAT: Meadows, rocky areas, alpine and subalpine.

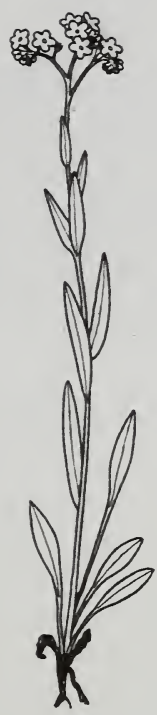

Myriophyllum sibiricum MYSI

shortspike watermilfoil Haloragaceae

HABIT: Stem from elongated, creeping rhizome.

KEY CHARACTERS: Pinnate leaves, usually forming a whorl. Spike emerging above water. Female flower has small petals, male flower has larger petals.

HABITAT: Shallow water.

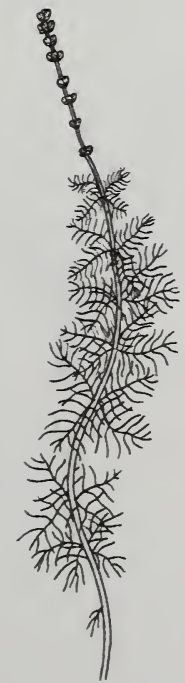


Nuphar polysepala yellow pondlily
NUPO2

Nymphaeaceae

HABIT: Stout herbaceous plant from submerged rhizomes.

KEY CHARACTERS: Leaves with petioles to 40 inches long or longer, the blades floating to emergent, leathery, 3-10 inches long, 4-9 inches wide. Flowers yellowish to purple.

HABITAT: Ponds and slowly flowing streams.

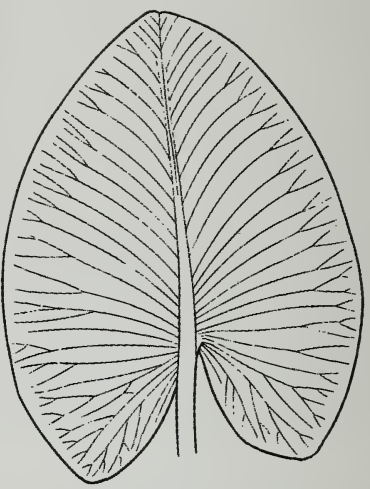

Osmorhiza depauperata blunt-fruit sweet-cicely

OSDE

Umbelliferae

HABIT: 6-28 inches.

KEY CHARACTERS: Leaves alternate, 1-3 times ternate, the leaflets again lobed and toothed. Flowers greenish-white or white, in umbel. Fruits club-shaped, hairy at base, $1 / 2-1$ inch long.

HABITAT: Forests.
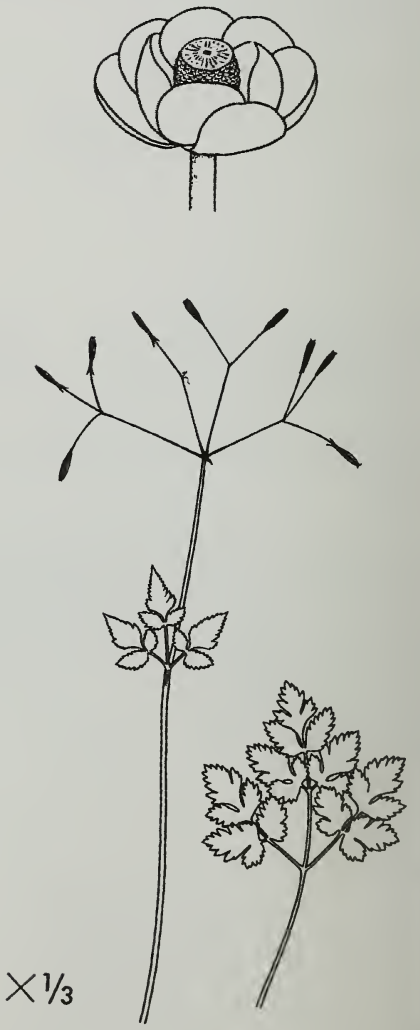

Osmorhiza purpurea
OSPU
Sitka sweet-cicely
Umbelliferae

HABIT: $12-40$ inches.

KEY CHARACTERS: Leaves alternate, 1-3 times-ternate, the leaflets again lobed and toothed. Flowers green to purple, in umbel. Fruits widest at middle, hairy, shorter than $1 / 2$ inch.

HABITAT: Forests, steambanks.

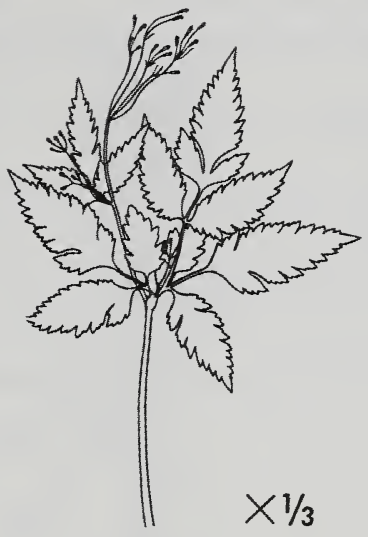

Oxytropis nigrescens blackish oxytrope
OXNI

Leguminosae

HABIT: Tufted to loosely matted.

KEY CHARACTERS: Leaves with 5-15 leaflets, leaflets hairy on both sides. Inflorescences with usually 2-3 flowers, petals purplish to blue.

HABITAT: Alpine tundra, heath, stony

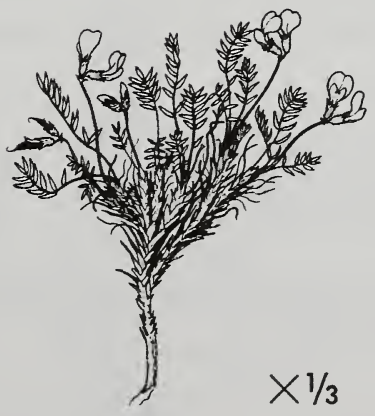
slopes. 
Parnassia palustris

PAPA8

northern grass-of-parnassus Saxifragaceae

HABIT: 4-18 inches.

KEY CHARACTERS: Leaves mostly basal, with short petioles. Flowers white, 1-several per plant, solitary, on long 1-leaved stem. Fruit capsules.

HABITAT: Heaths, wet meadows.

Pedicularis capitata capitate lousewort
PECA2

Scrophulariaceae

HABiT: Single stem, 2-6 inches tall.

KEY CHARACTERS: Leaves pinnately compound, the leaflets lobed or dissected. Inflorescence in a head of 1-8 flowers, petals cream colored.

HABITAT: Alpine tundra, heath, rocky slopes.
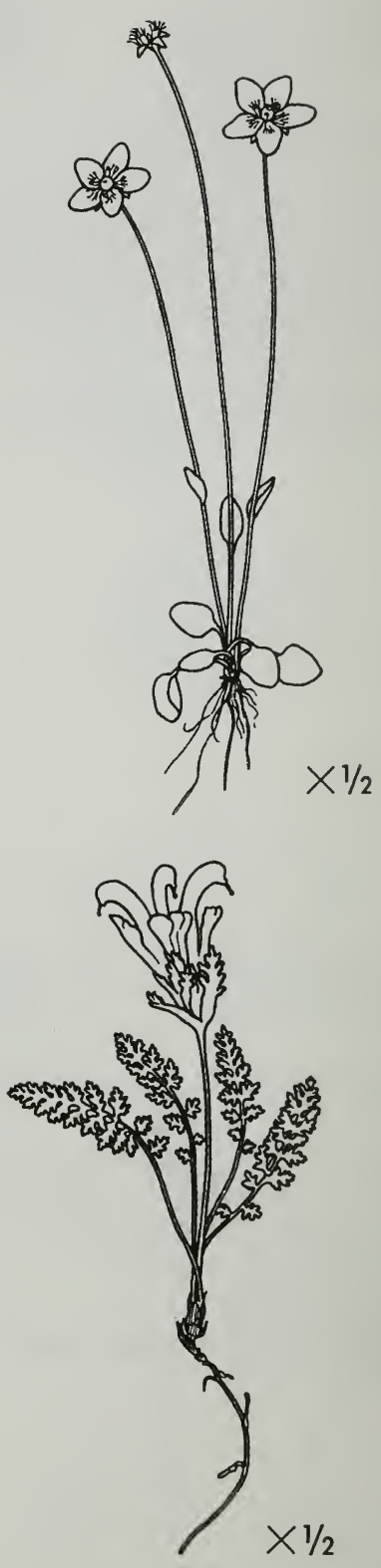
Pedicularis kanei woolly lousewort
PEKA7

Scrophulariaceae

HABIT: Single stem, hairy in the inflorescence, 2-10 inches tall.

KEY CHARACTERS: Leaves pinnately compound, the leaflets dissected into many segments. Inflorescence elongated and many flowered, petals rose pink to lavender.

HABITAT: Alpine tundra, heath, rocky areas.

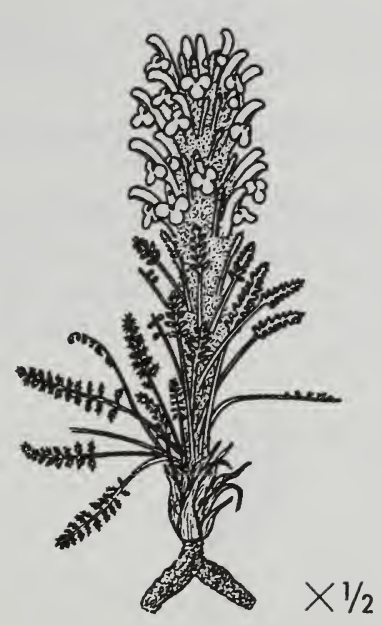

Pedicularis labradorica PELA

Labrador lousewort Scrophulariaceae

HABIT: Branched or unbranched, 6-10 inches.

KEY CHARACTERS: Leaves alternate, pinnately divided. Stem with white hairs. Flowers yellow or reddish, several per plant, upper petal forming hood enclosing stamens. Fruit capsules.

HABITAT: To alpine. Forests.

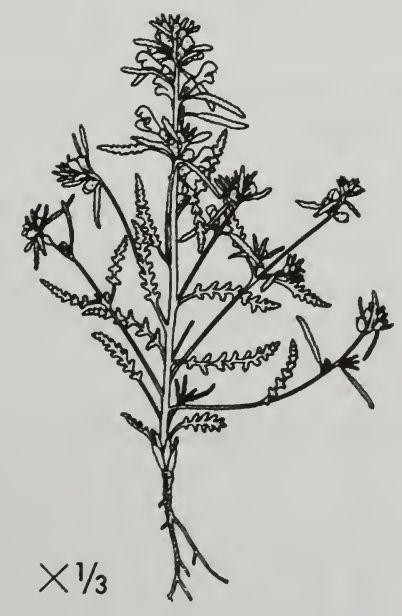


Pedicularis langsdorfii Langsdorf's lousewort
PELA3

Scrophulariaceae

HABIT: Single stem, 2-9 inches tall.

KEY CHARACTERS: Leaves lobed to pinnatifid, the lobes toothed or smooth.

Inflorescence in an elongated head, several to many-flowered, flowers rose pink to pink-purple.

HABITAT: Dry to moist tundra and heathlands.

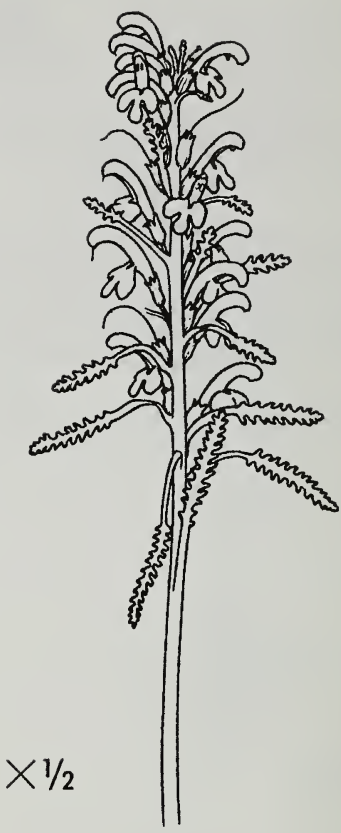

Pedicularis parviflora

PEPA4

smallflower lousewort Scrophulariaceae

HABIT: Simple stem, or mostly branched from the base.

KEY CHARACTERS: Leaves along stem oblong and deeply pinnately lobed, with dentate segments. Flowers in 2 parts, rose-colored, lower lip 3-lobed and broader than long.

HABITAT: Swamps, muskeg.

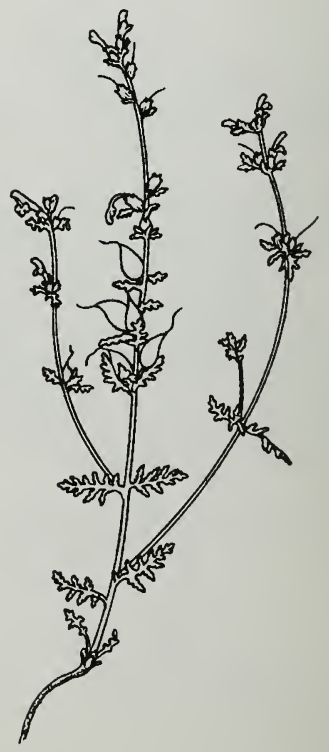


HABIT: Stems single or several, 3-16 inches tall.

KEY CHARACTERS: Basal leaves with long petioles. Stem leaves with short petioles or sessile, whorled. All leaves pinnatifid, the lobes toothed or incised. Inflorescence in a head or elongate with many flowers, flowers rose pink.

HABITAT: Alpine tundra, heath, meadows, woods, rocky slopes.

\section{Petasites hyperboreus \\ PEHY5 \\ arctic sweet coltsfoot \\ Compositae}

HABIT: Simple stems, from creeping rootstocks, with reddish scales.

KEY CHARACTERS: Leaves deeply lobed into 3-5 broad, grossly toothed segments, smooth above with white fuzz beneath. Flowers purplish.

HABITAT: Wet tundra, shores, along creeks.
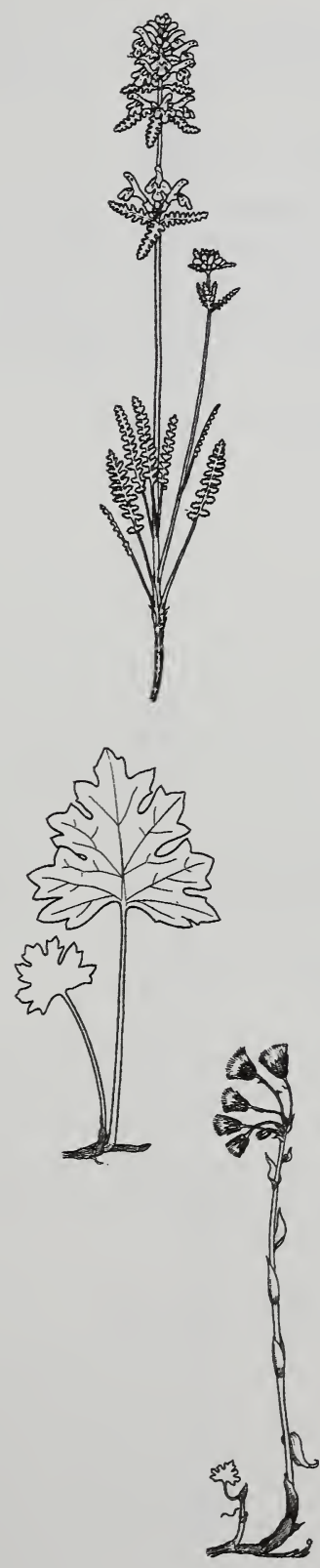
Pinguicula vulgaris common butterwort

PIVU

Lentibulariaceae

HABIT: 3-7 inches.

KEY CHARACTERS: Leaves in basal rosette, shiny, fleshy. Flowers blue to violet, longer than $1 / 2$ inch, 1-2 per plant, solitary, on long leafless stems, nodding, with blunt or pointy spur. Fruit spherical capsules.

HABITAT: Wet areas.

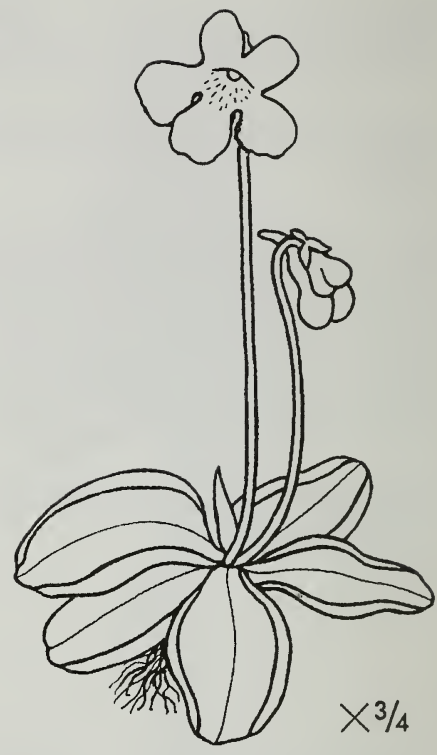

Plantago maritima goosetongue plantain
PLMA3

Plantaginaceae

HABIT: $3-7$ inches.

KEY CHARACTERS: Leaves linear-lanceolate in basal rosette, shiny, fleshy. Flowers blue to violet, longer than 1 inch, 1-2 per plant, solitary, on long leafless stems, nodding, with blunt or pointy spur. Fruit spherical capsules.

HABITAT: Wet areas.

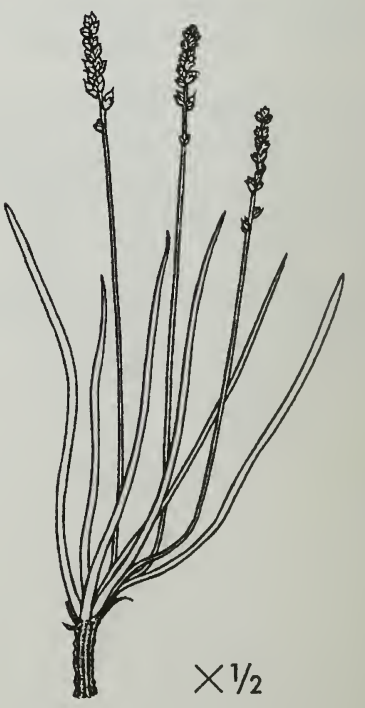


HABIT: Stems from fleshy, rootlike tuberoids.

KEY CHARACTERS: Leaves ovate to lanceolate. Many-flowered spike with white flowers, sweet-scented.

HABITAT: Wet meadows, bogs.

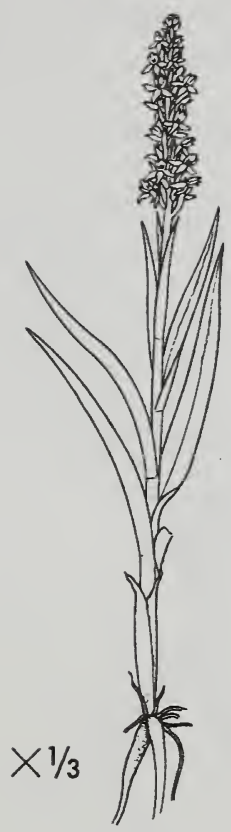

Platanthera saccata

slender bog orchid
PLSA6

Orchidaceae

HABIT: Upright, 6-20 inches.

KEY CHARACTERS: Stems with several leaves, oblanceolate to lanceolate. Inflorescence a few to many-flowered raceme. Flowers greenish.

HABITAT: Open woods, thickets, muskegs, meadows.

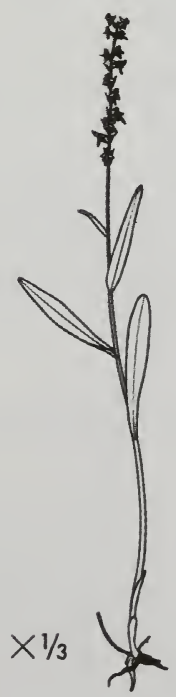


Polemonium acutiflorum tall Jacob's-ladder
POAC

Polemoniaceae

HABIT: 8-40 inches.

KEY CHARACTERS: Leaves alternate, with several hairless pinnately compound leaflets. Stem hairy. Flowers blue to violet, several per plant, with pointy, hairy-edged petals. Fruit round capsules.

HABITAT: Streambanks, wet meadows.

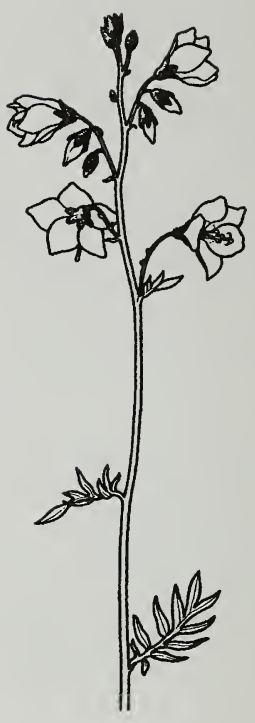

Polygonum viviparum

POVI3

alpine bistort

Polygonaceae

HABIT: Thick, hard, usually contorted rhizome.

KEY CHARACTERS: Leaves from base lanceolate-oblong, shiny above, grayish below, smooth. Spike with lower flowers replaced by bulblets with white or pink flowers.

HABITAT: Dry meadows, heaths, to at least 7000 feet.

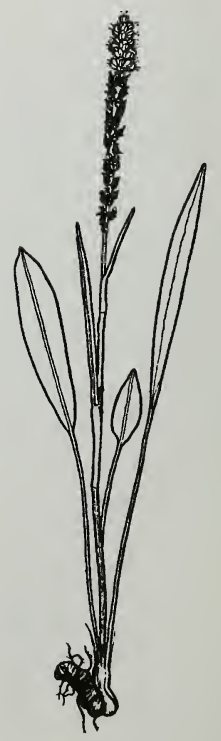


Potamogeton filiformis POFI2

slender-leaved pondweed Potamogetonaceae

HABIT: Threadlike stem, branched especially at the base.

KEY CHARACTERS: Bristlelike leaves, brownish-green and blunt. Long flower stalk, spike with 3-4, usually widely separated whorls of flowers.

HABITAT: Shallow water.

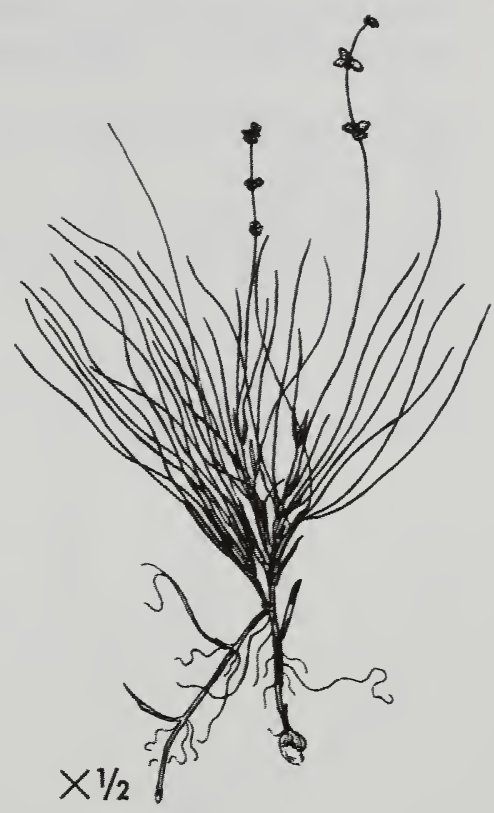

Potamogeton natans

PONA4

floating pondweed

Potamogetonaceae

HABIT: Rounded stem up to 40 inches long.

KEY CHARACTERS: Submerged leaves linear with no differentiation between the blade and the petiole. Floating leaves up to $3 \frac{1}{2}$ inches long and $1 \frac{1}{2}$ inches wide, thick, with many prominent parallel veins. Green fruits on a spike $1 \frac{1}{2}-2$ inches long.

HABITAT: Lakes, ponds, streams with sluggish water.

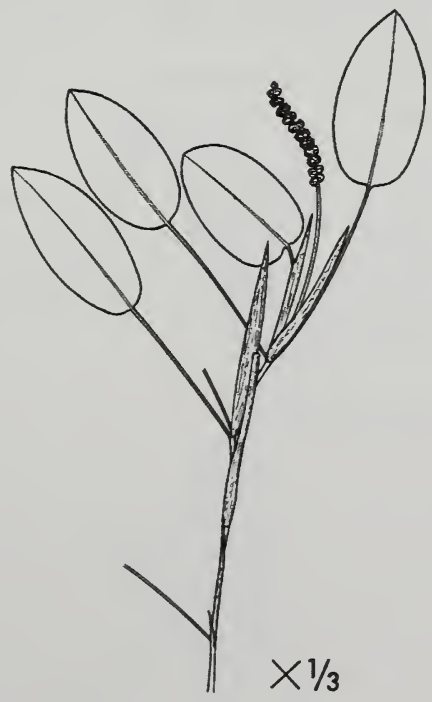


Potamogeton pectinatus

POPE6

sago pondweed

Potamogetonaceae

HABIT: Rounded stem up to 15 inches long.

KEY CHARACTERS: Leaves all submerged, hairlike. Fruits in about 5 whorls on a spike.

HABITAT: Lakes and ponds (often with saline or brackish water).

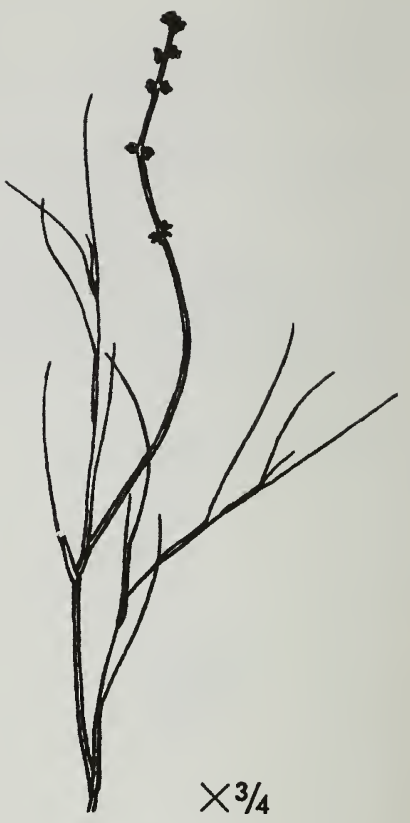

Potamogeton perfoliatus POPER5

claspingleaf pondweed Potamogetonaceae

HABIT: Rounded stem up to 40 inches long.

KEY CHARACTERS: All leaves submerged. Stem densely covered with ovate, dark-green leaves, often clasping the stem all around. Short, curved flower stalk with a brown spike.

HABITAT: Lakes.

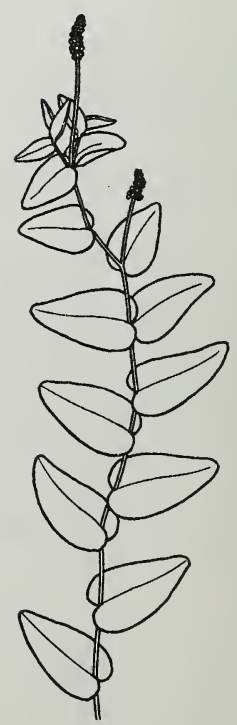




\section{Potentilla egedii}

Pacific silverweed

POEG

Rosaceae

HABIT: Hairy stolons.

KEY CHARACTERS: All leaves are at or near the base, compound, completely or almost completely smooth, with 2-5 pairs of leaflets. Single-flowered with yellow petals.

HABITAT: Often found in wet places.
Potentilla palustris marsh fivefinger
POPA14

Rosaceae
HABIT: Prostrate to ascending, 4-40 inches.

KEY CHARACTERS: Leaves toothed, pale and pubescent underneath, with 5-7 leaflets. Flowers brownish-purple, several per plant. Fruit achenes.

HABITAT: Wet meadows, streams, shallow water.
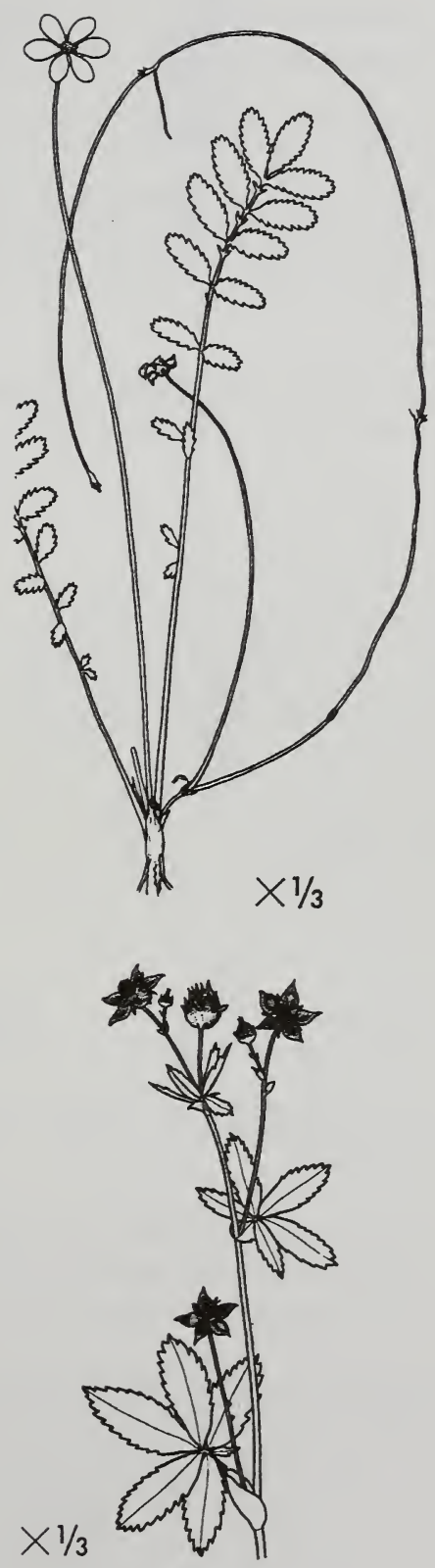
Prenanthes alata rattlesnake root
PRAL

Compositae

HABIT: 8-30 inches.

KEY CHARACTERS: Leaves alternate, white below, arrowhead-shaped, toothed. Flowers white, several per plant. Fruit achenes.

HABITAT: Streambanks, wet open areas, beach fringe, subalpine forests.

Primula cuneifolia wedgeleaf primrose
PRCU

Primulaceae

HABIT: Stem up to 3 inches long. Leaves all basal.

KEY CHARACTERS: Leaves thick, 5-11 teeth at apex. Inflorescence an umbel with 1-9 flowers. Petals pink to rose or white.

HABITAT: Alpine tundra, heath, wet meadows.
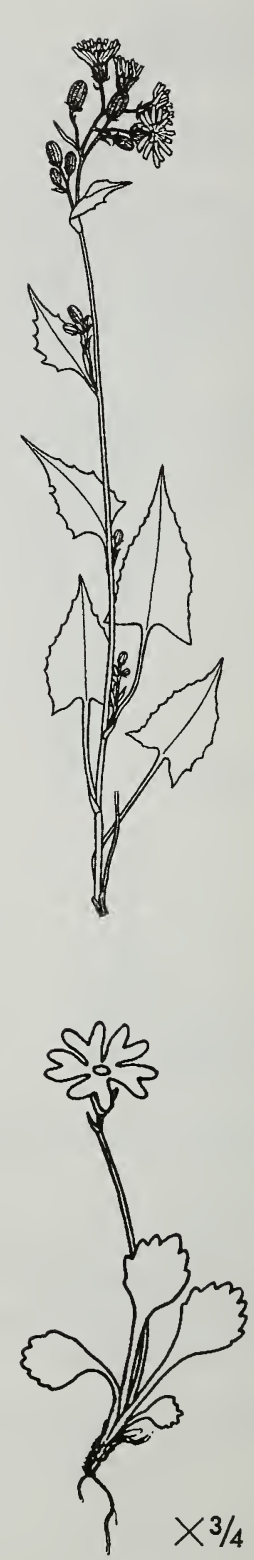

Pyrola asarifolia
PYAS
liverleaf wintergreen
Pyrolaceae

HABIT: Perennial, 5-16 inches.

KEY CHARACTERS: Leaves basal, purplish underneath, rounded, toothed, leathery. Flowers crimson to pink, nodding, several in 1-3 bracted, long-stemmed terminal raceme, nodding, style long and curved. Fruit capsules.

HABITAT: Forests, gravelly open areas, beach forests.

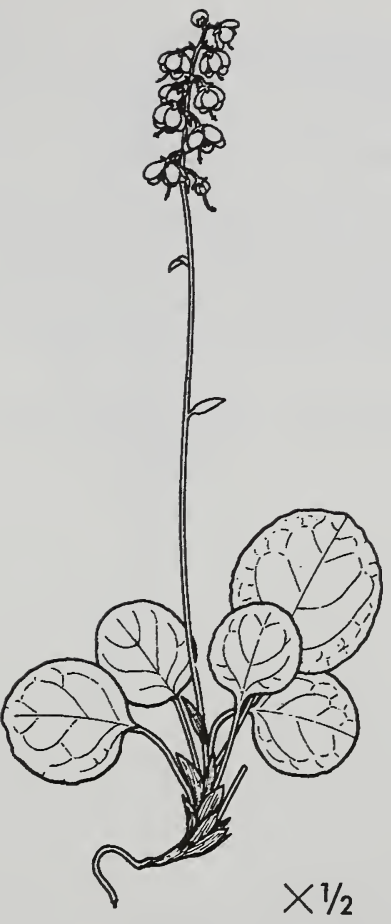

Pyrola chlorantha

greenish wintergreen
PYCH

Pyrolaceae

HABIT: Perennial, $1 / 2-1$ inch.

KEY CHARACTERS: Leaves basal, small, round, toothed, leathery. Flowers pale yellowish or greenish-white, few-10 in long leafless raceme, style curved. Fruit capsules.

HABITAT: Forests.

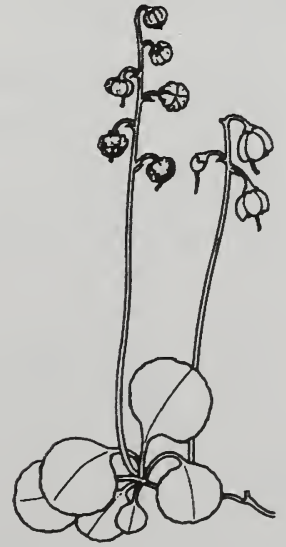


Pyrola grandiflora

largeflowered wintergreen

PYGR

Pyrolaceae

HABIT: $2 \frac{1}{2}-5$ inches.

KEY CHARACTERS: Leaves basal, the blades thick, lustrous, rounded.

Inflorescence a 4-11 flowered raceme.

Petals white or greenish-white.

HABITAT: Alpine tundra, heath, woods.

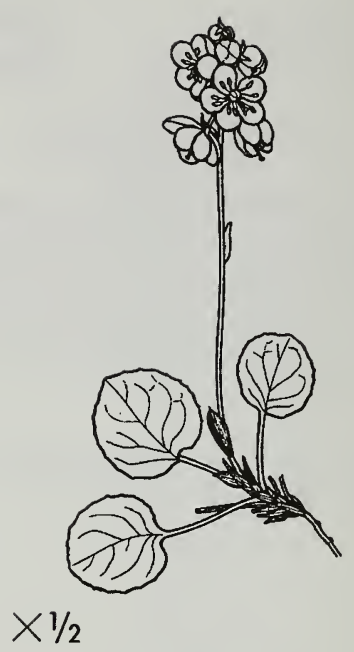

Pyrola minor

snowline wintergreen

PYMI

Pyrolaceae

HABIT: $2 \frac{1}{2}-5$ inches.

KEY CHARACTERS: Leaves basal. Inflorescence a 5-13 flowered raceme. Petals white or pinkish.

HABITAT: Meadows, heath, woods.

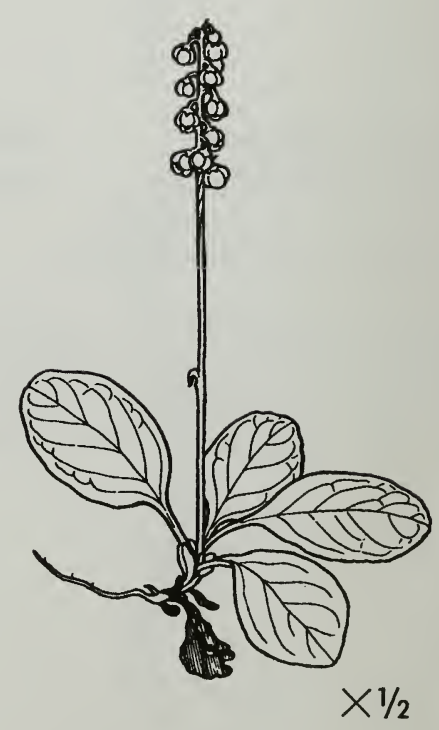



Pyrola secunda
PYSE
one-sided wintergreen
Pyrolaceae

HABIT: Perennial, 3-8 inches.

KEY CHARACTERS: Leaves mostly basal, toothed. Flowers greenish-white, several in 1-5 bracted long-stemmed one-sided inflorescence. Fruit capsules.

HABITAT: Open forests.

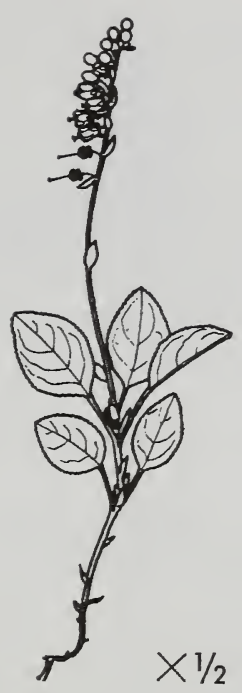

Ranunculus cymbalaria seaside buttercup

HABIT: Tufted, threadlike stolons.

KEY CHARACTERS: Rounded, kidney-or heart-shaped leaves. Small flowers with usually 5 yellow petals. Ovate to cylindrical fruiting head and a hairy receptacle.

HABITAT: Moist places, brackish water, sometimes apparently spread by human activity.

RACY

Ranunculaceae

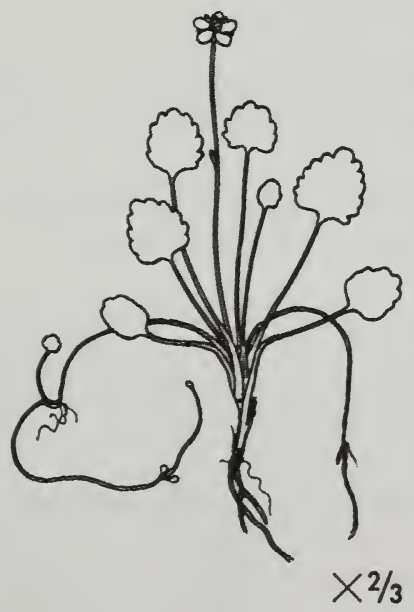


Ranunculus occidentalis RAOC

western buttercup Ranunculaceae

HABIT: 3-25 inches.

KEY CHARACTERS: Basal leaves 3-parted, the parts lobed or toothed. Stem leaves alternate, with no petiole. Flowers yellow, several per plant, solitary. Fruit achenes in hemispherical head.

HABITAT: Moist meadows, tundra.

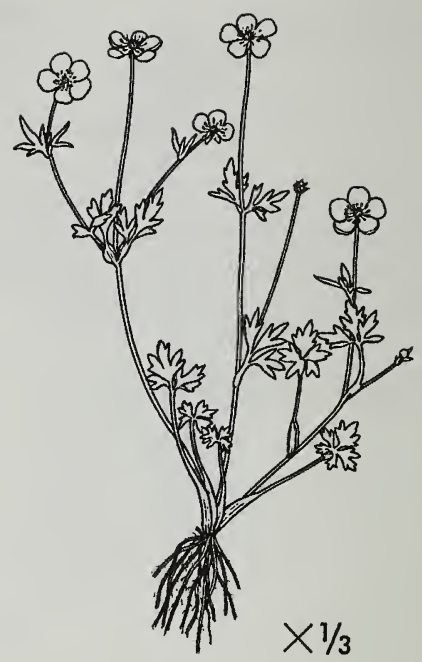

Ranunculus trichophyllus RATR

white water crowfoot Ranunculaceae

HABIT: Aquatic, stems floating or submerged, 8-80 inches long.

KEY CHARACTERS: Leaves dark green, finely dissected into hairlike segments, the upper leaves palmately divided into 3-5 lobes. Flowers white.

HABITAT: Ponds and streams.

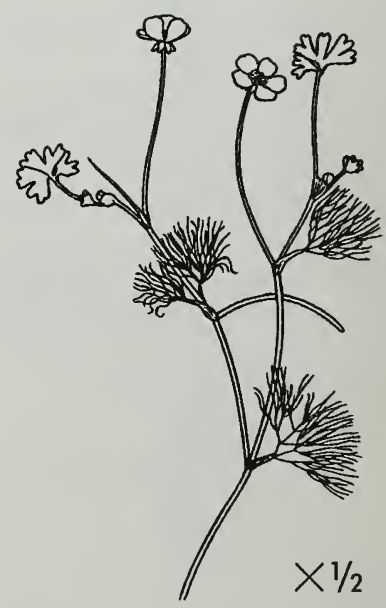


Rhinanthus minor yellow rattle
RHMI13

Scrophulariaceae

HABIT: Somewhat branched or unbranched, to 30 inches.

KEY CHARACTERS: Leaves opposite, narrow, toothed. Flowers yellow, numerous. Fruit capsules.

HABITAT: Meadows, disturbed areas, beaches.

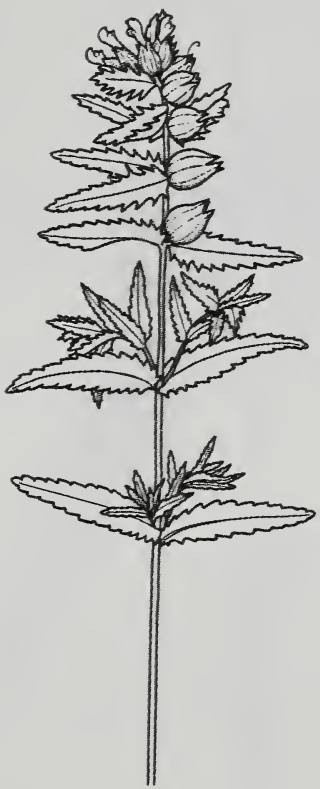

Rubus arcticus nagoonberry

HABIT: 1-6 inches.

KEY CHARACTERS: Leaves alternate, palmately compound or 3-lobed. Flowers pink, few per plant, usually solitary, terminal, with narrow petals. Fruit red to purplish.

HABITAT: Forests, meadows, muskegs, streambanks, beach fringe.

RUAR

Rosaceae
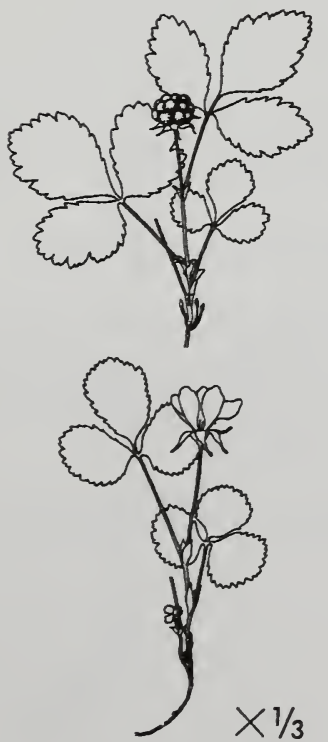
Rubus chamaemorus cloudberry
RUCH

Rosaceae

HABIT: 2-12 inches.

KEY CHARACTERS: Leaves roundish, 3-5 lobed, toothed, leathery. Flowers white, solitary, terminal. Fruits yellow-orange when ripe.

HABITAT: Meadows, muskegs, alpine.

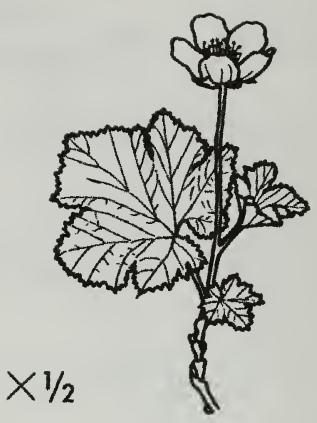

Rubus pedatus

fiveleaf bramble
RUPE

Rosaceae

HABIT: Trailing, 1-4 inches.

KEY CHARACTERS: Leaves toothed, with 5 palmately compound leaflets.

Flowers white, solitary, on few-bracted stalk. Fruits red.

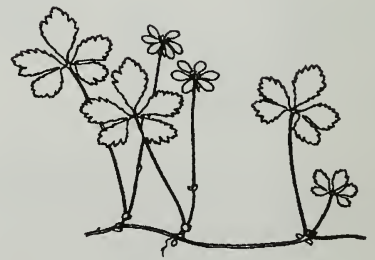

$\times 1 / 3$

HABITAT: Forests, meadows, muskegs. 
Rumex fenestratus western dock
RUFE3

Polygonaceae

HABIT: Upright from taproots, 20-80 inches, usually unbranched below the inflorescence.

KEY CHARACTERS: Lower leaves up to 12 inches long, the upper leaves smaller. Inflorescence a dense panicle.

HABITAT: Marshes, beaches, stream banks, tidal flats.

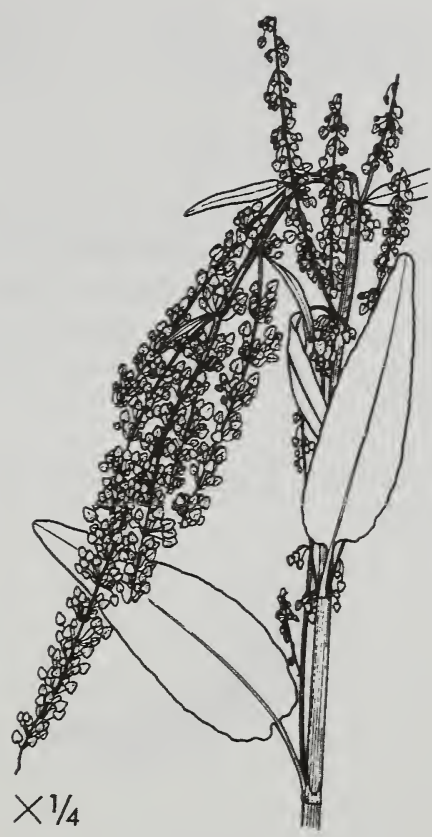

Sanguisorba stipulata

SAST11

Sitka burnet

Rosaceae

HABIT: 10-30 inches.

KEY CHARACTERS: Leaves pinnately compound, toothed. Flowers

greenish-white, in a dense, long-stemmed, 1-3 inch spike with 1-3 reduced leaves, stamens very long and conspicuous. Fruit achenes.

HABITAT: Meadows, muskegs, swamps, tundra.

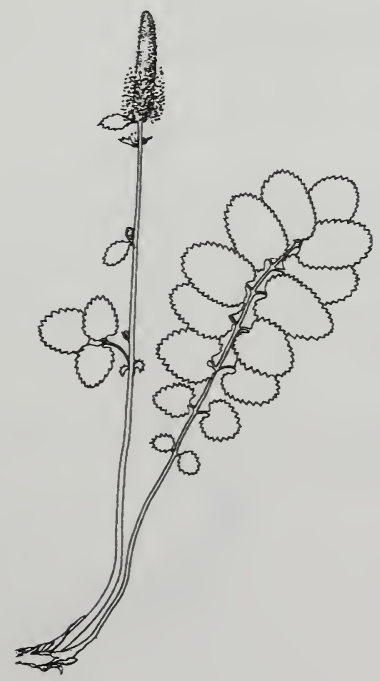


Saxifraga bronchialis

yellowdot saxifrage
SABR6

Saxifragaceae

HABIT: 1-6 inches.

KEY CHARACTERS: Leaves leathery, with no petiole, pointed tips, and stout hairs on edges. Flowers white or cream, spotted with yellow or red, in several-leaved long-stemmed inflorescence. Fruit capsules.

HABITAT: Rocky soil, rock crevices, cliffs, tundra.

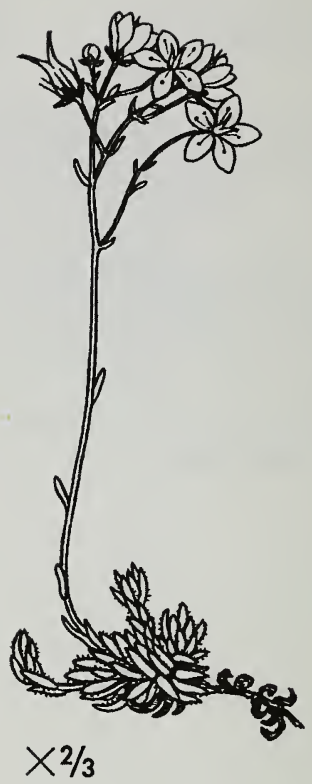

Saxifraga punctata

heart-leaved saxifrage
SAPU6

Saxifragaceae

HABIT: 2-24 inches.

KEY CHARACTERS: Leaves

kidney-shaped, with many large teeth.

Flowers white to pink, in leafless

long-stemmed inflorescence. Fruit capsules.

HABITAT: Streambanks, open areas, alpine meadows.

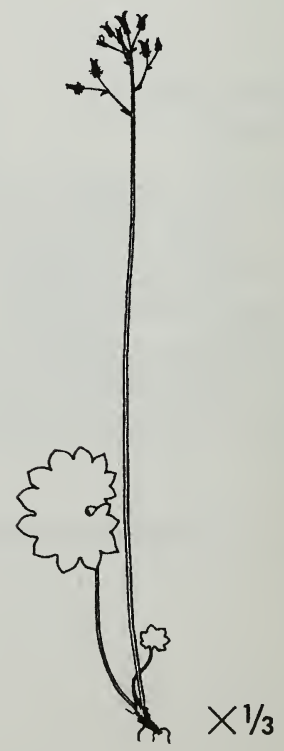


HABIT: Evergreen, 1-10 inches.

KEY CHARACTERS: Leaves brownishgreen, leathery, with 3 teeth and hairy edges. Flowers white with yellow spots at base and red spots at tip, in long-stemmed inflorescence with several reduced leaves. Fruit capsules.

HABITAT: Gravelly slopes, rock crevices, cliffs, ridges, tundra.

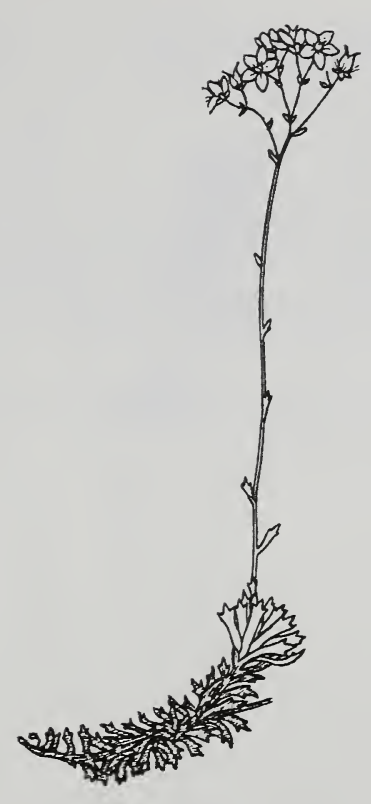

Sedum rosea

roseroot stonecrop
SEROI3

Crassulaceae

HABIT: Upright from thick rhizome, 3-10 inches.

KEY CHARACTERS: Stem with many leaves. Leaves reduced and scalelike on the lower stem, becoming larger higher up. Flowers purple.

HABITAT: Scree slopes, rocky places, alpine tundra, heath, open woods.

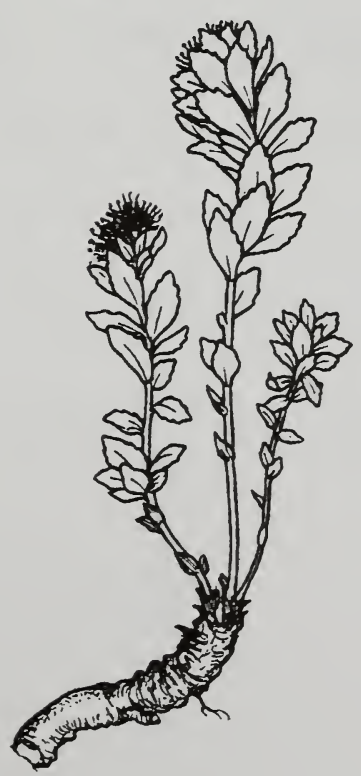


Senecio triangularis arrowleaf groundsel

SETR

Compositae

HABIT: $1-4$ feet.

KEY CHARACTERS: Leaves alternate, triangular, toothed. No basal leaves. Flowers yellow, several in flat-topped inflorescences. Fruit hairless achenes.

HABITAT: Streambanks, wet meadows, open areas.

Solidago multiradiata northern goldenrod
SOMU

Compositae

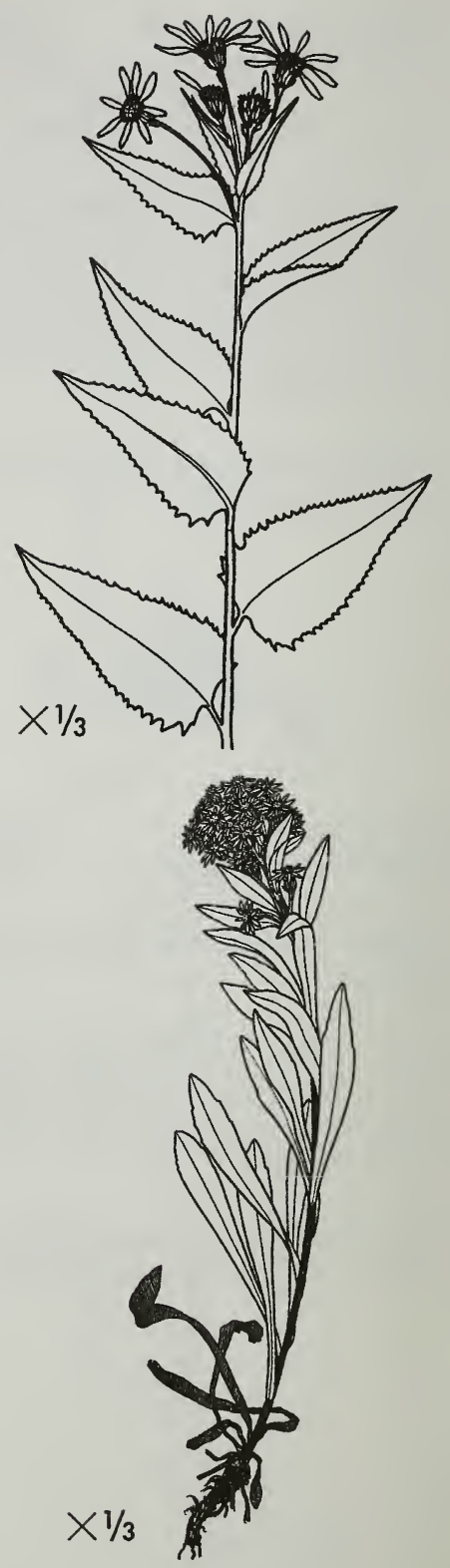

HABIT: Upright, 4-20 inches.

KEY CHARACTERS: Leaves alternate, toothed, with hairy edges at base. Flowers small, yellow, several in dense inflorescence. Fruit hairy achenes.

HABITAT: From lowlands to the lower alpine. Meadows, open areas, rocky soil. 
Sparganium angustifolium SPAN2

floating burreed

Sparganiaceae

HABIT: Aquatic plant with stems 6-25 inches long.

KEY CHARACTERS: Leaves usually floating, distinctly named. Fruit with pointed beak longer than $1 / 20$ inches.

HABITAT: Ponds and sluggish streams.

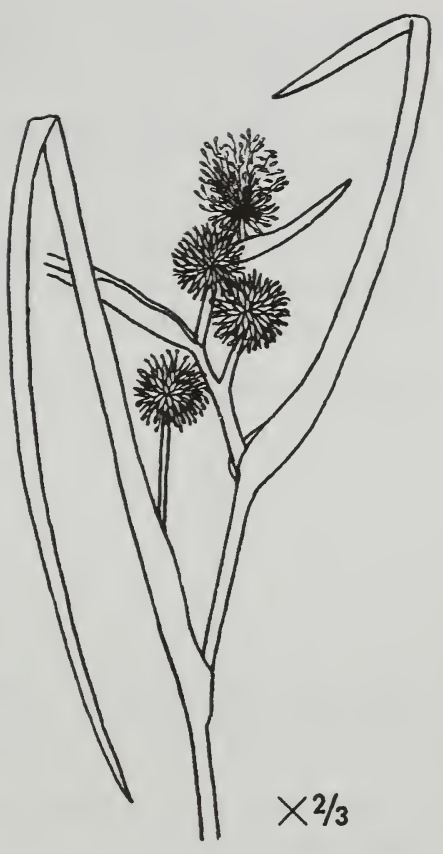

Sparganium hyperboreum SPHY northern burreed Sparganiaceae

HABIT: Aquatic plant with stems 4-10 inches long.

KEY CHARACTERS: Leaves upright or floating. Fruit nearly beakless.

HABITAT: Ponds and lakes.

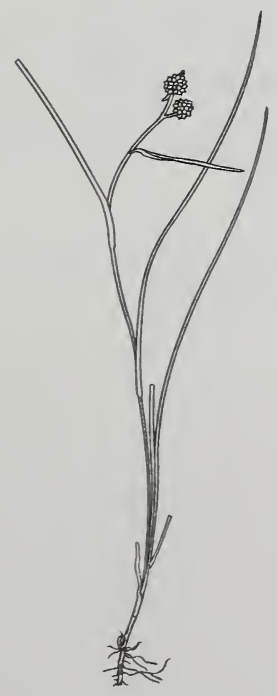


Sparganium minimum

small burreed
SPMI

Sparganiaceae

HABIT: Aquatic plant with stems up to 15 inches long.

KEY CHARACTERS: Leaves upright or floating, lacking median nerve. Fruit with short cone-shaped beak.

HABITAT: Ponds and lakes.

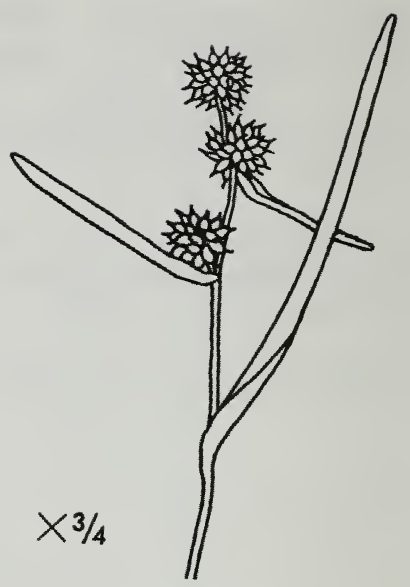

Spiranthes romanzoffiana SPRO hooded ladies' tresses

Orchidaceae

HABIT: Stem leafy in lower part, from fleshy tuberoid roots.

KEY CHARACTERS: Flowers in 3 spiral rows, fragrant and white to creamy. Sepals and 2 petals forming hood, lip constricted above the middle with a terminal round to ovate lobe.

HABITAT: Bogs, marshes, in mountains to at least 3000 feet.

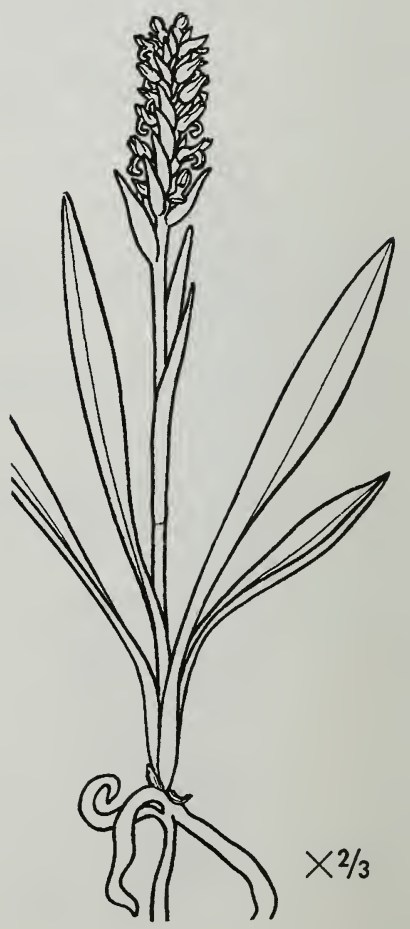


Stellaria calycantha northern starwort
STCA

Caryophyllaceae

HABIT: Weak stem, curving upward, branching, smooth or slightly rough.

KEY CHARACTERS: Leaves without stalks, opposite, ovate-lanceolate, pointed. Single flowers in forks of stem.

HABITAT: Wet places.

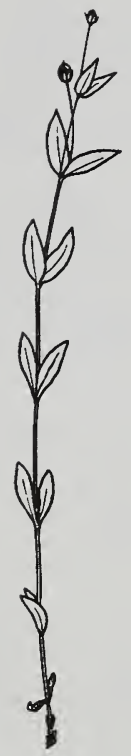

Stellaria crassifolia fleshy starwort
STCR

Caryophyllaceae

HABIT: Forms mats or clumps, stems 1-10 inches long.

KEY CHARACTERS: Leaves opposite, without petiole, mostly much longer than broad. Flowers $1-3$ in open cymes, petals white.

HABITAT: Muskegs, open woods, lake shores, snow beds.

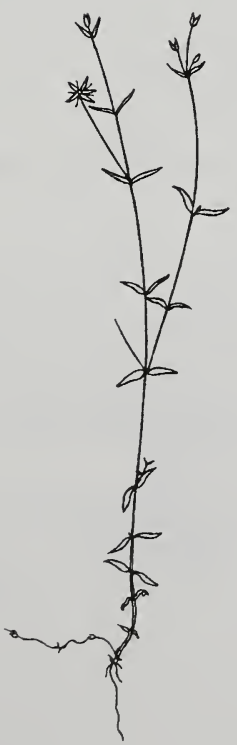


HABIT: Forms mats, stems 4-20 inches long.

KEY CHARACTERS: Leaves opposite, without petiole, egg-shaped in outline, sharply pointed at the apex. Flowers solitary in leaf axils, petals usually lacking.

HABITAT: Wet soil in woods, on stream banks, and on beaches.

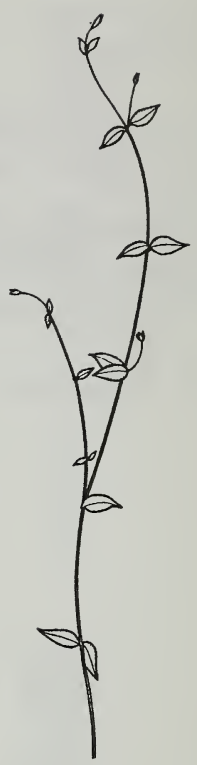

Stellaria sitchana STSI3

Sitka starwort Caryophyllaceae

HABIT: Forms clumps, stems up to 20 inches long.

KEY CHARACTERS: Leaves opposite, without petiole, mostly much longer than broad. Inflorescences mostly open cymes with several to many flowers, petals usually absent (white when present).

HABITAT: Wet meadows, river banks, thickets, open woods.

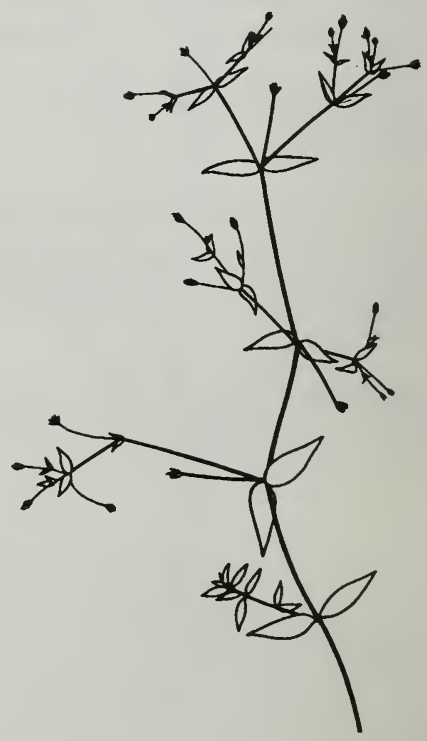


Streptopus amplexifolius twistedstalk
STAM2

Liliaceae

HABIT: Upright, branched, 10-40 inches.

KEY CHARACTERS: Leaves alternate, clasping stem, with prominent veins.

Flowers white, 1-2 per stalk, from leaf bases. Fruit red berries.

\section{HABITAT: Forests.}

Subularia aquatica awlwort

SUAQ

Cruciferae

HABIT: Aquatic, often submerged, stem up to 4 inches tall.

KEY CHARACTERS: Leaves linear and tapering to a sharp point. Flowers very small, petals white.

HABITAT: Ponds, streams, shores.
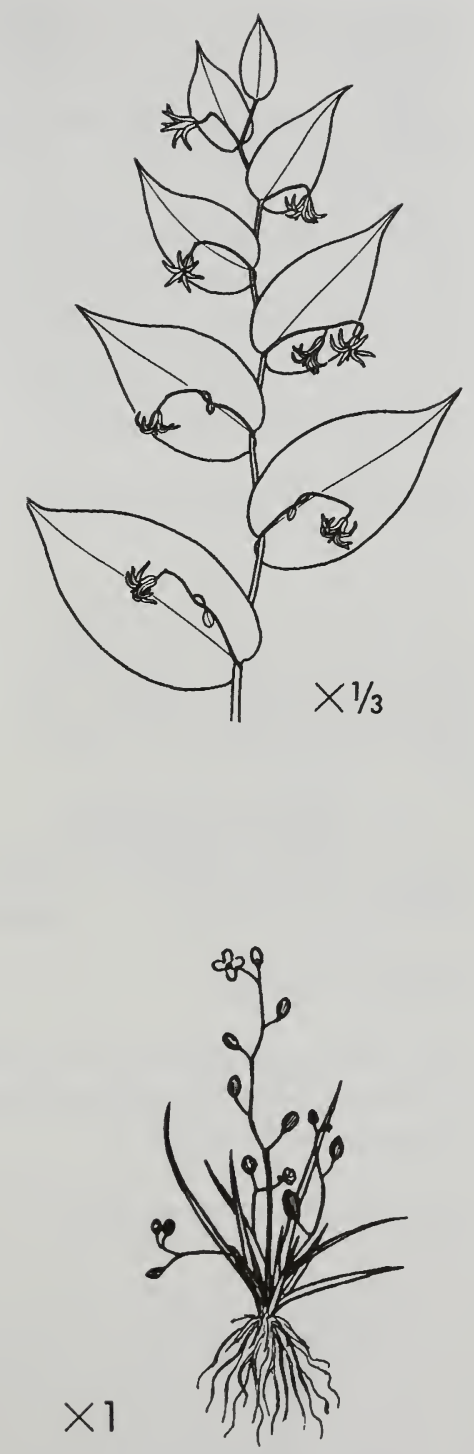
HABIT: Smooth, straight stem, simple, from woody base.

KEY CHARACTERS: Leaves at base long-stalked, elliptic to oblong, entire. Upper stem leaves not stalked, alternate or in pairs, somewhat pointed. Flowers 5-lobed and pointed, grayish-blue, mottled, variable in color, rarely white.

HABITAT: Subalpine meadows.

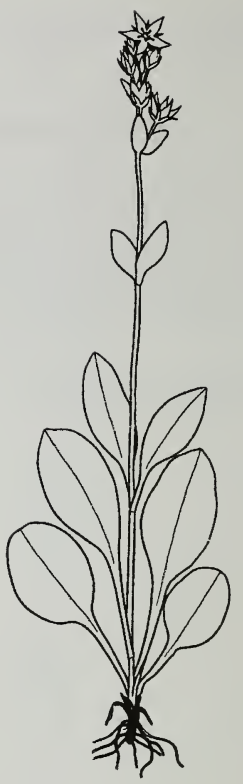

Thalictrum sparsiflorum THSP

fewflower meadowrue Ranunculaceae

HABIT: To 4 feet.

KEY CHARACTERS: Leaves alternate, 2-3 times ternate. Flowers pinkish-white, no petals. Fruit achenes.

HABITAT: Forests, meadows.

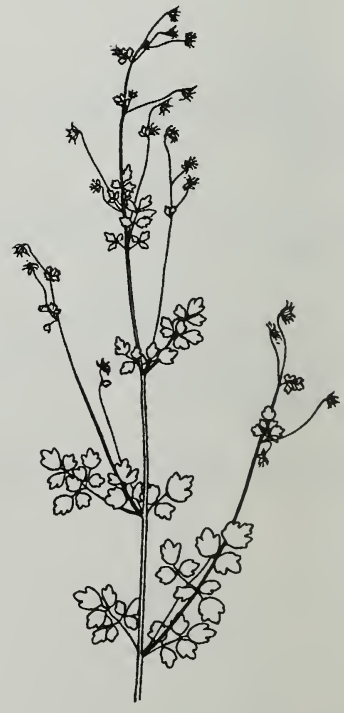


Tiarella trifoliata foamflower
TITR

Saxifragaceae

HABIT: Perennial, 6-20 inches.

KEY CHARACTERS: Leaves mostly basal, toothed, with 3 leaflets. Flowers white, in 1-2 leaved long-stemmed inflorescence. Fruit sugarscoop-like capsules.

HABITAT: Forests, meadows, seeps, floodplains.

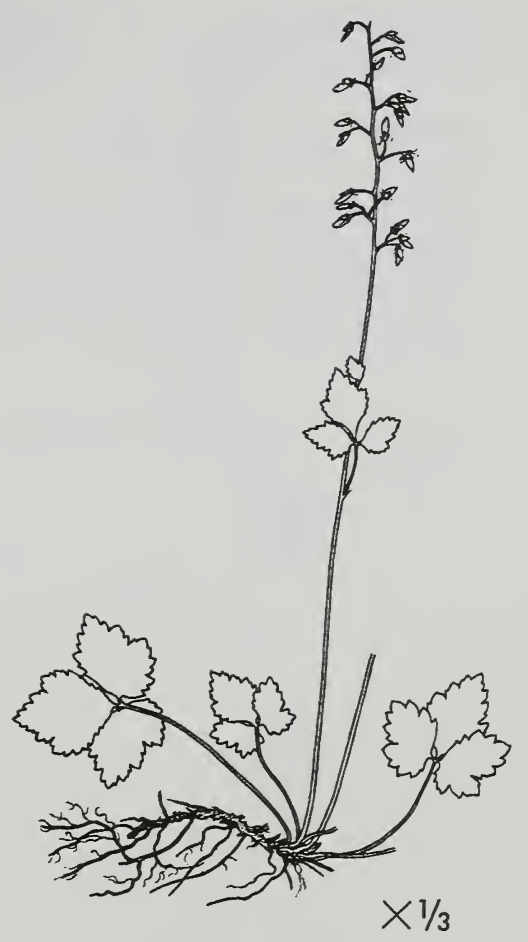

Tofieldia coccinea TOCO northern asphodel Liliaceae

HABIT: Tender, 2-4 inches.

KEY CHARACTERS: Leaves mostly basal, slender, on 2 sides of stem. Stems not sticky. Flowers yellowish, in spike-like raceme. Fruit capsules.

HABITAT: Alpine tundra, heath, stony areas.

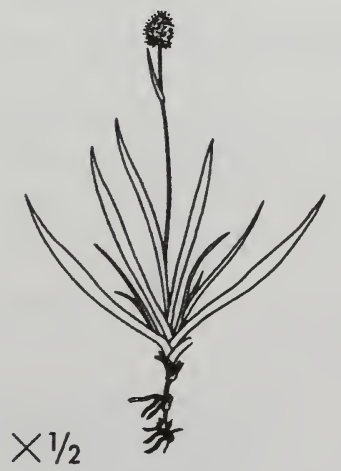


Tofieldia glutinosa

sticky false-asphodel
TOGL2

Liliaceae

HABIT: Stout, 6-12 inches.

KEY CHARACTERS: Leaves mostly basal, slender, on 2 sides of stem. Stems sticky. Flowers yellowish, in leafless to 1-bracted long-stemmed spike-like raceme. Fruit capsules.

HABITAT: Open forests, meadows, muskegs.

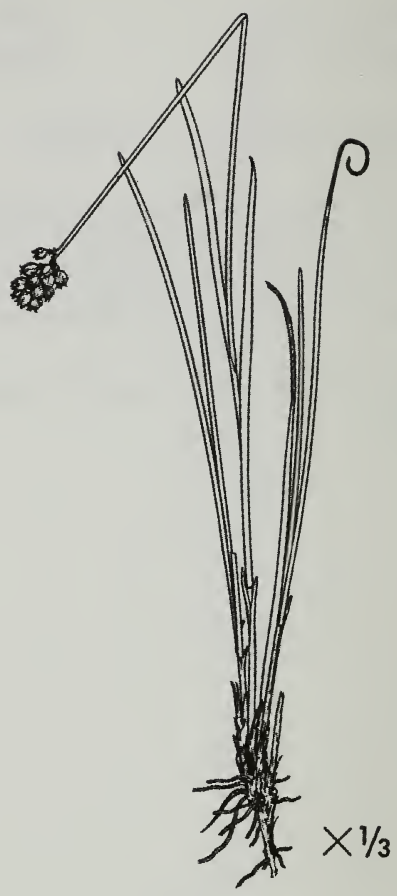

Trientalis europaea starflower

TREU

Primulaceae

HABIT: Unbranched, upright, 2-6 inches.

KEY CHARACTERS: Leaves 5-6 in terminal whorl with much smaller, alternate stem leaves below. Flowers white, 1-3 per plant, solitary, terminal. Fruit capsules.

HABITAT: Forests, meadows, muskegs, open areas.

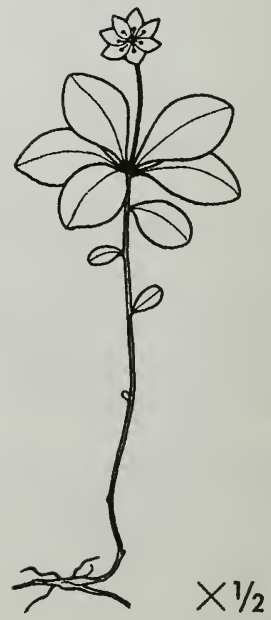


Triglochin maritimum seaside arrowgrass
TRMA4

Juncaginaceae

HABIT: 5-35 inches.

KEY CHARACTERS: Leaves all basal, fleshy, linear, from thick woody rhizome. Inflorescence a several to many-flowered raceme. Flower segments greenish or yellowish.

HABITAT: Muskegs, saline meadows, tidal flats, open woods.

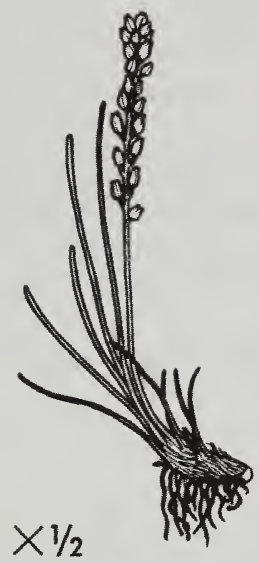

Utricularia vulgaris common bladderwort

UTVU

Lentibulariaceae

HABIT: Free floating.

KEY CHARACTERS: Leaves much dissected, the leaf segments hair like. Leaves all with bladders. Flowers 5-15, yellow.

HABITAT: Ponds and lakes.

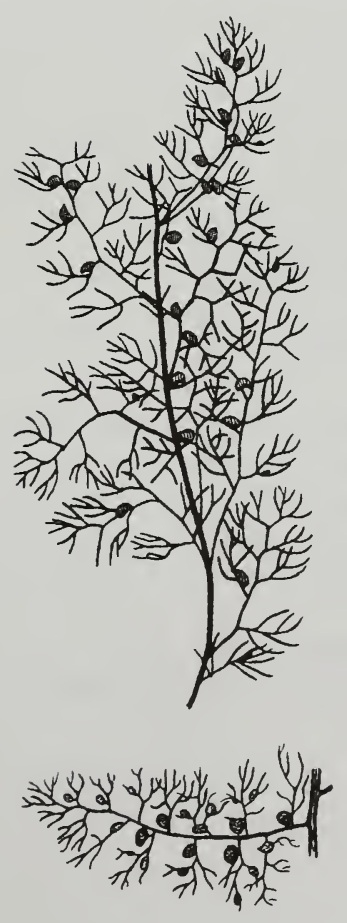


Valeriana sitchensis

Sitka valerian
VASI

Valerianaceae

HABIT: $1-3$ feet.

KEY CHARACTERS: Leaves mostly on stem, opposite, toothed, with 3-5 leaflets. Flowers white or lilac, in dense terminal inflorescence. Fruit achenes.

HABITAT: Moist places, tundra.

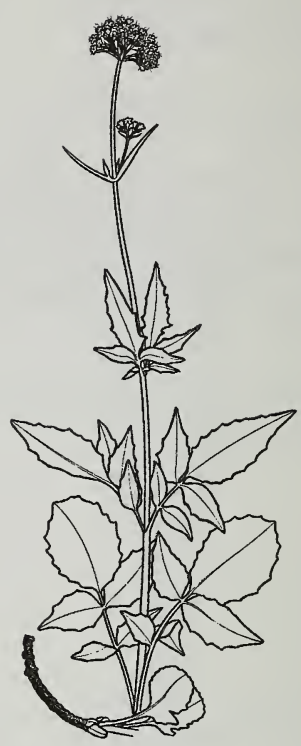

Veratrum viride

VEVI

false hellebore

Liliaceae

HABIT: 3-8 feet.

KEY CHARACTERS: Leaves alternate, broad, with parallel veins, woolly pubescent underneath. Flowers yellowish-green, in terminal inflorescence with long, hanging branches. Fruit 3-parted capsules.

HABITAT: Meadows, moist places, shores in Prince William Sound.

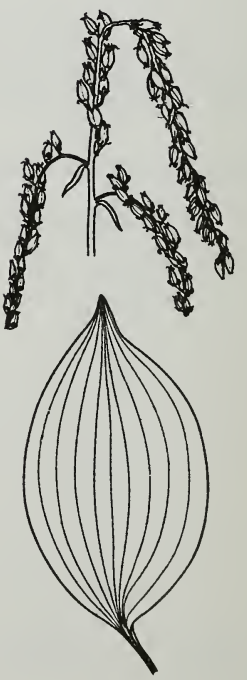


Viola epipsila marsh violet
VIEP

Violaceae

HABIT: 2-10 inches.

KEY CHARACTERS: Leaves basal, toothed, shiny, thin, with rounded tip.

Flowers lilac, solitary, on long stem with pair of bracts, nodding, with short and thick spur. Fruit capsules.

HABITAT: Muskegs, steambanks, wet meadows, open areas.

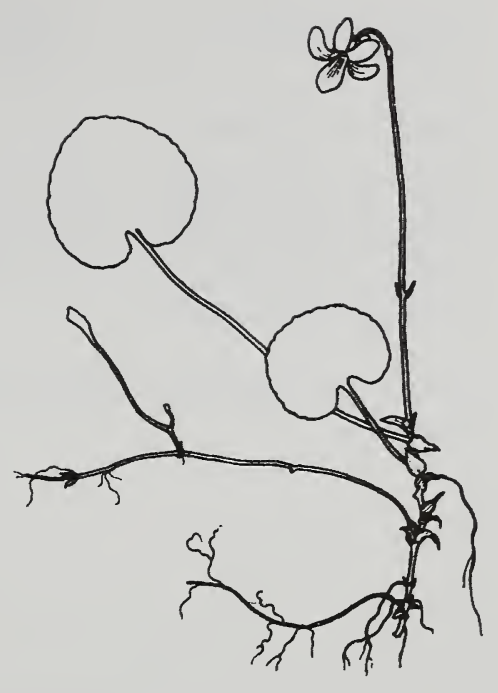

$\times 1 / 2$

Viola glabella yellow violet

HABIT: 2-12 inches.

KEY CHARACTERS: Basal leaves toothed, with pointed tip. Stem leaves opposite. Flowers yellow, solitary, nodding. Fruit capsules.

HABITAT: Moist forests, riverbanks.

VIGL

Violaceae

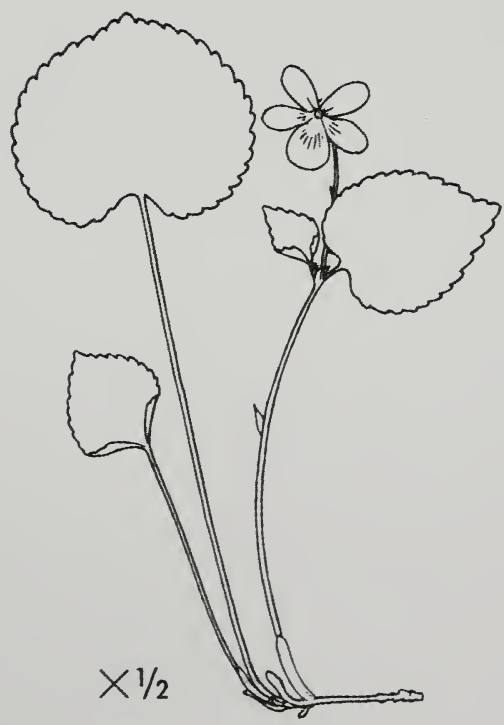


Viola langsdorffii

Alaska violet
VILA6

Violaceae

HABIT: 2-10 inches.

KEY CHARACTERS: Leaves basal, toothed, with rounded tip. Flowers purple, solitary, on long stem with pair of bracts, nodding. Fruit capsules.

HABITAT: Steambanks, moist meadows, snowbeds.
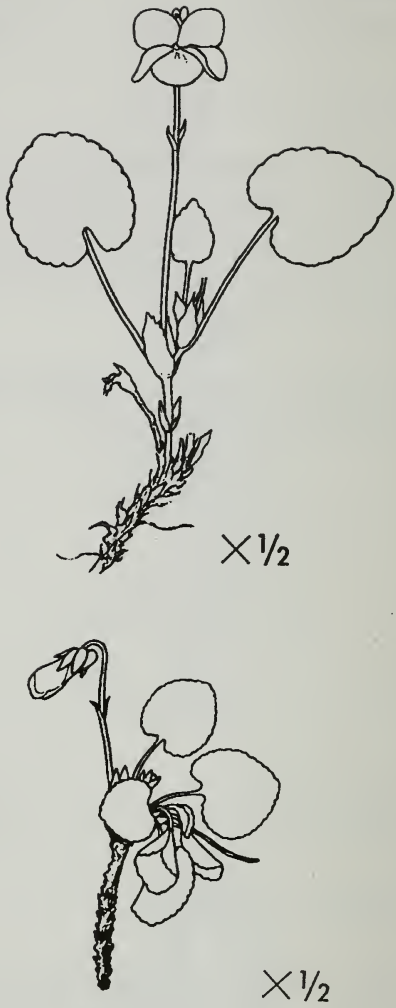
GRAMINOIDS 


\section{SEDGES/CYPERACEAE}

Stems triangular, 3-ranked (leaves on 3

sides of stem) and mostly solid. Fruit usual-

ly an achene, never a caryopsis. 1 bract

below flowers. Anthers attached at base.

\section{GRASSES/GRAMINEAE}

Stems round or flattened, 2-ranked (leaves on 2 sides of stem), and hollow. Fruit usually a caryopsis. 2 bracts below flowers.

Anthers attached centrally.

\section{RUSHES/JUNCACEAE}

Stems round and mostly solid, 3-ranked.

Flowers usually have a well-developed perianth of six segments. Fruit capsular with numerous seeds. Petals and sepals present. 
Agrostis aequivalvis arctic bentgrass
AGAE

Gramineae

Refer to Podagrostis aequivalis in Hultén (1968).

HABIT: Perennial, rhizomatous, forming small tufts, to 30 inches.

KEY CHARACTERS: Inflorescence a 2-6 inch long, loose, open panicle. Spikelets with 1 flower. Lemmas shorter than glumes, awnless.

HABITAT: Bogs and lake margins.

Agrostis alaskana

Alaska bentgrass
AGAL2

Gramineae

HABIT: Perennial, shortly rhizomatous, forming small to large tufts, to 30 inches.

KEY CHARACTERS: Inflorescence a 1-6 inch long, narrow to open panicle. Spikelets with 1 flower. Lemmas shorter than glumes, awnless or awned.

HABITAT: Bogs, lake shores, sea beaches.
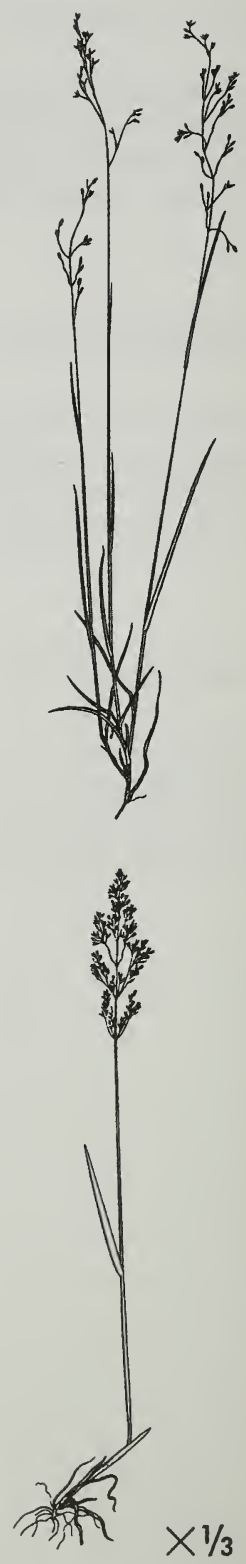
Arctagrostis latifolia polargrass
ARLA2

Gramineae

HABIT: Perennial, rhizomatous, tufted or solitary, 1-5 feet.

KEY CHARACTERS: Few basal leaves. Inflorescence a 2- to 26-inch long purplish open or short-branched panicle. Spikelets with 1 flower. Lemmas finely hairy, longer than glumes, awnless.

HABITAT: Forests, meadows, gravel bars, tundra.

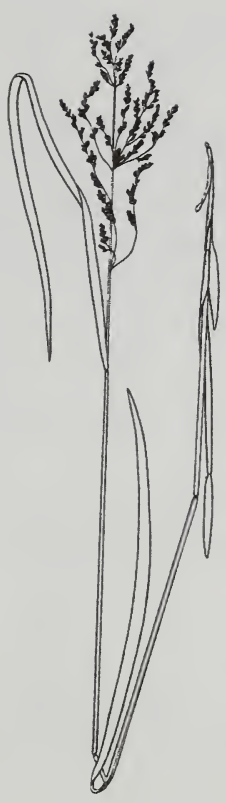

Arctophila fulva

pendant grass
ARFU2

Gramineae

HABIT: Perennial, rhizomatous, 8-30 inches tall.

KEY CHARACTERS: Panicle open, the branches recurved or drooping, 2-9 inches long. Spikelets with 1-7 flowers, glumes shorter than lemmas.

HABITAT: Lake and pond margins, stream banks.

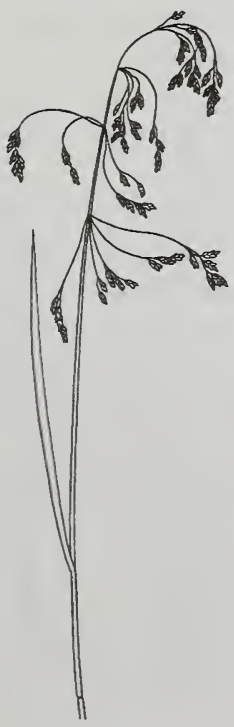


Calamagrostis canadensis bluejoint reedgrass
CACA4

Gramineae

HABIT: Rhizomatous, to 6 feet.

KEY CHARACTERS: Inflorescence a 2-9 inch long open panicle. Spikelets with 1 flower. Florets with hairs longer than lemmas. Lemmas distinctly to barely shorter than glumes, with straight or curved dorsal awn, the awn shorter to slightly longer than glumes. Glumes usually purplish.

HABITAT: Open forests, meadows, shrublands, open areas, beaches.

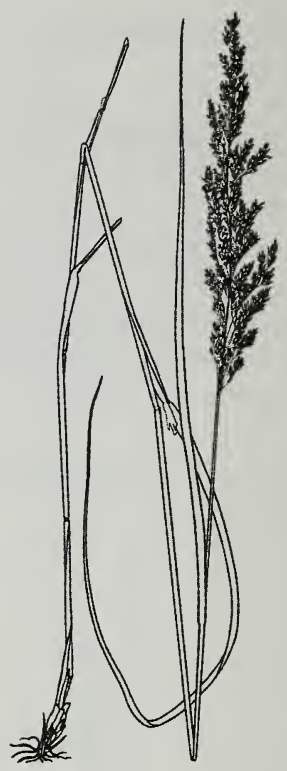

Calamagrostis nutkaënsis

Pacific reedgrass

CANU

Gramineae

HABIT: Rhizomatous, tufted, to 3 feet.

KEY CHARACTERS: Inflorescence a 4-8 inch long narrow greenish or purplish open panicle. Spikelets with 1 flower. Florets with hairs not more than half as long as lemmas. Lemmas shorter than glumes, with awn straight or bent at base.

HABITAT: Wet places along coast.

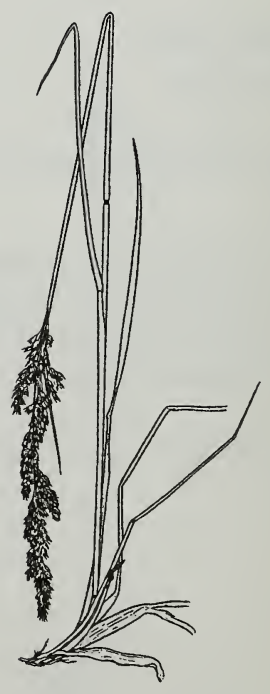


Carex anthoxanthea arctic sedge
CAAN10

Cyperaceae

HABIT: Culms with bladeless sheaths at base, 2-5 inches tall, in rows from creeping, yellowish-brown rhizome.

KEY CHARACTERS: Solitary spike, smooth, fusiform perigynia, yellowishgreen.

HABITAT: Grassy slopes.

Carex aquatilis water sedge
CAAQ

Cyperaceae

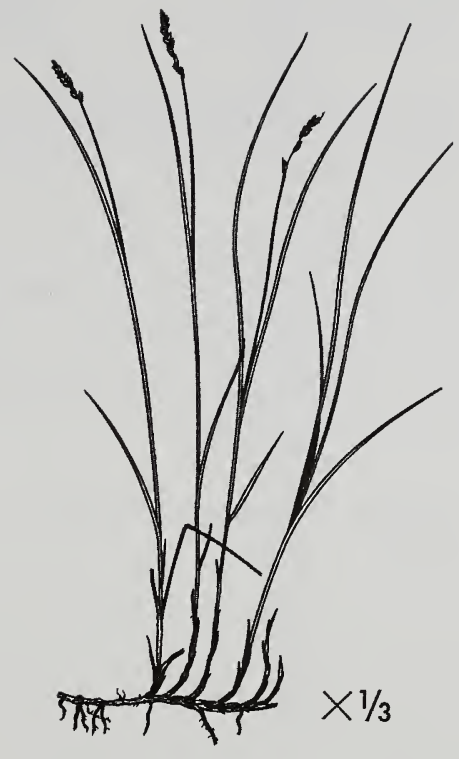

HABIT: Height to 30 inches. Cordlike rhizome, coarse, scaly, and brown or reddish-brown.

KEY CHARACTERS: Leaves as long as stem, or slightly shorter. Stiff, erect spikes, the upper sessile, the lower short-stalked. Scales brown or blackish with a pale midvein. Light colored perigynia.

HABITAT: Shallow water, marshes, and along rivers.

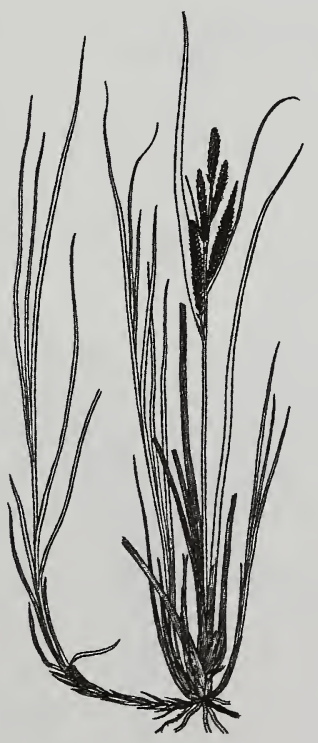


Carex lyngbyaei

Lyngbye's sedge
CALY3

Cyperaceae

HABIT: Stoloniferous, tufted to 3 feet tall. Triangular, smooth culm, longer than leaves.

KEY CHARACTERS: Flat leaves, light green and abruptly pointed. Male spikes $2-3$, female 2-4. Inflorescences drooping, long-stalked. Lanceolate scales, long-pointed, dark reddish-brown with a lighter center. Ovate perigynia.

HABITAT: Coastal salt marshes, rarely inland.

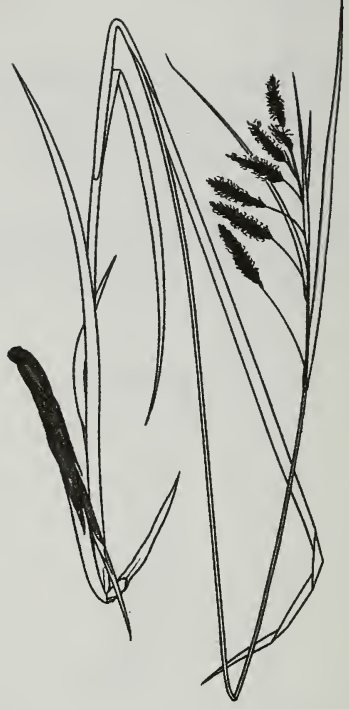

Carex macrochaeta longawned sedge CAMA11 Cyperaceae

HABIT: Loosely in tufts or dense clumps, with short runners to 3 feet tall. Culm longer than leaves, nearly smooth, with purplish-brown, bladeless sheaths, shredded at the base.

KEY CHARACTERS: Light green leaf blades. Oblong spikes $3-4$, on thin, smooth stalks. Lanceolate scales with a light-colored midvein, excurrent into yellowish awn. Elliptic-lanceolate perigynia.

HABITAT: Wet places, common along coast, rarer inland.

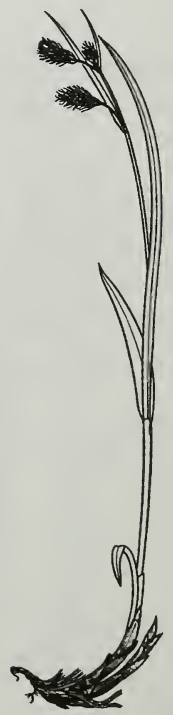


Carex magellanica

boreal bog sedge
CAMA 12

Cyperaceae

HABIT: Rhizomatous and more or less casepitose, 4-30 inches tall.

KEY CHARACTERS: Roots with yellowish woolly hairs. Leaves flat, pale green, shorter than the culm. Terminal spike mostly staminate, the lateral 2-4 pistillate on short, drooping, stalks. Pistillate scales longer than the perigynia, lanceolate, coming to a long point.

HABITAT: Bogs, muskegs, lake shores.

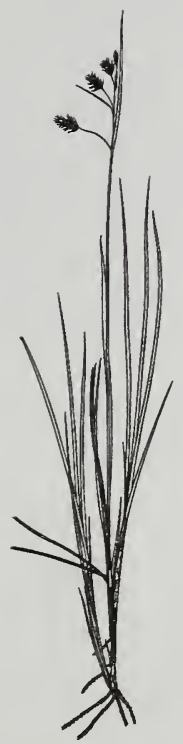

Carex microchaeta

CAMI4

smallawned sedge

Cyperaceae

HABIT: Long, slender, fibrous rootstock. Culms with many basal leaves, 3-25 inches tall, the outer withered and brown.

KEY CHARACTERS: Leaves much shorter than culm. Spikes 2-4, in mature plants drooping. Acute scales, purplish-black with lighter midrib. Elliptic perigynia, blackishtinged.

HABITAT: Meadows, wet places, heaths to over 6900 feet.

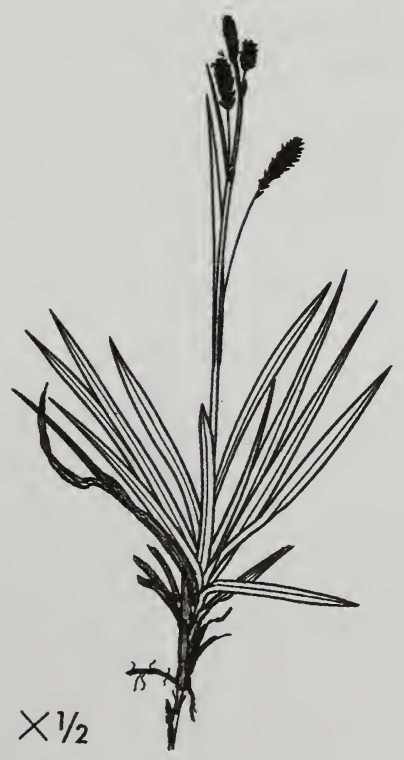


Carex microglochin

fewseeded bog sedge
CAMI6

Cyperaceae

HABIT: Rhizomatous, $2 \frac{1}{2}-8$ inches tall.

KEY CHARACTERS: Leaf sheaths brownish, lower leaf blades short, blades longer higher on culm. Spikes solitary, staminate flowers at tip. Pistillate scales shorter than the perigynia. Perigynia abruptly reflexed.

HABITAT: Marshy heath, lake shores, stream banks.

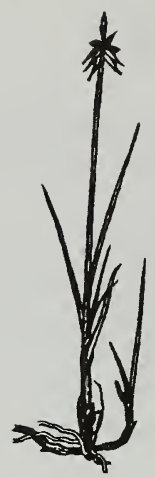

$\times 2 / 3$

Carex pauciflora fewflower sedge
CAPA19

Cyperaceae

HABIT: Loosely tufted with short, brown, scaly runners. Culm curved at base, 4-10 inches.

KEY CHARACTERS: Lowest sheath bladeless. Style projecting beyond the mouth of brownish-green perigynia.

HABITAT: Peat bogs, prefers acid soil.

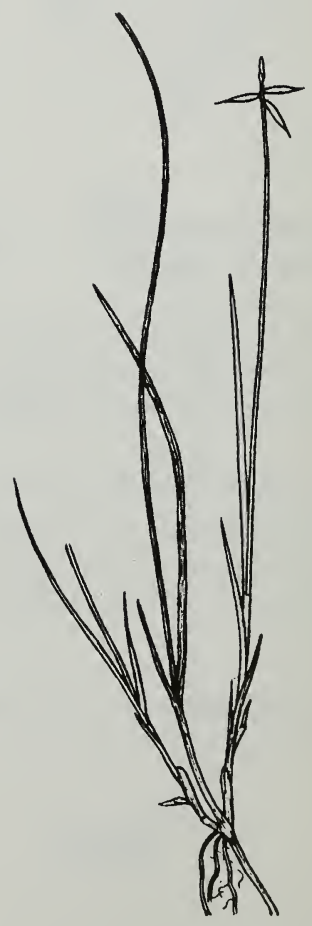


HABIT: Long, scaly, purplish-black rhizome, fuzzy roots. Triangular culm, 4-15 inches.

KEY CHARACTERS: Leaves about as long as culm, flat, grayish-green. Spikes $2-3$, the terminal male, long-stalked, the lateral female, drooping on hairlike stalks, 10-20 flowered. Scales broadly ovate and pointed at the tip, black, with a paler midvien. Ovate to ovate-lanceolate perigynia, in age blackish-brown.

HABITAT: Bogs and margins of ponds along coast.

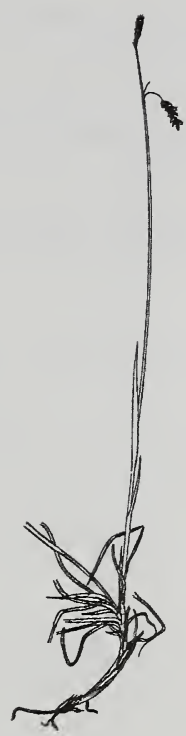

Carex sitchensis

CASI3

Sitka sedge

Cyperaceae

HABIT: Very coarse, short, scaly rhizome, brown or purplish. Culms sharply triangular and smooth, to 40 inches tall.

KEY CHARACTERS: Male spikes 2-5, slender, erect, female strongly separate, slightly drooping, on long, slender stalks. Scales brownish, with pale center, pointed in age, distinctly thin and translucent at tip. Oval perigynia.

HABITAT: Swamps.

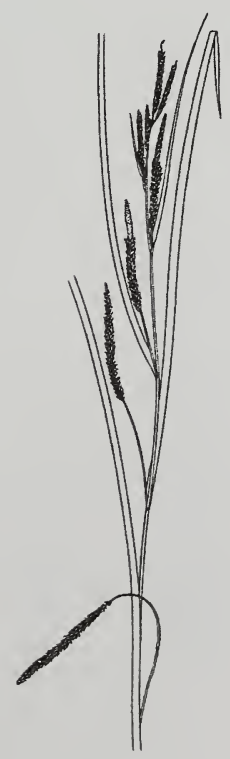


Deschampsia caespitosa DECE tufted hairgrass

Gramineae

Includes $D$. beringensis as defined by Hultén (1968).

HABIT: Tufted, up to and exceeding 3 feet tall.

KEY CHARACTERS: Smooth leaves, mostly flat. Open, branched flower cluster, with hairlike, very scabrous branches. Awn mostly fixed close to base, slightly longer than floret. Fuzzy rachis. Flower cluster typically yellowish-green, but many are darker.

HABITAT: Muddy shores.

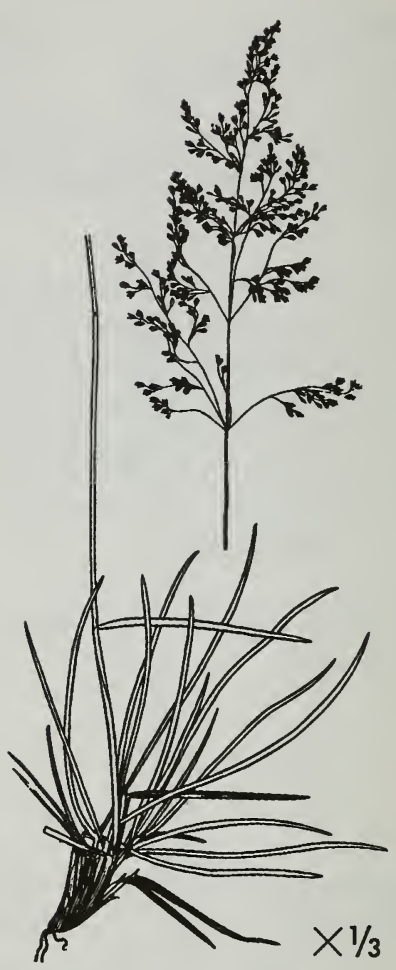

$\begin{array}{ll}\text { Eleocharis acicularis } & \text { ELAC } \\ \text { needle spikerush } & \text { Cyperaceae }\end{array}$

HABIT: Caespitose with slender rhizomes and stolons, to 4 inches tall.

KEY CHARACTERS: Leaves reduced to sheaths (no leaf blades). Spikes solitary.

HABITAT: Mud banks along streams, lakes, or ponds.

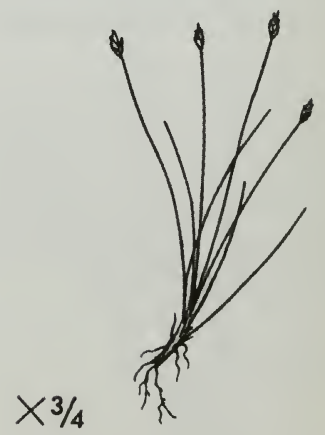


HABIT: Stiff culms, to 25 inches tall, with reddish basal sheaths from stout, brownish-black rhizome.

KEY CHARACTERS: Lanceolate to ovate spike, brown with two basal scales, each clasping half of the culm.

HABITAT: Wet places, shallow water.

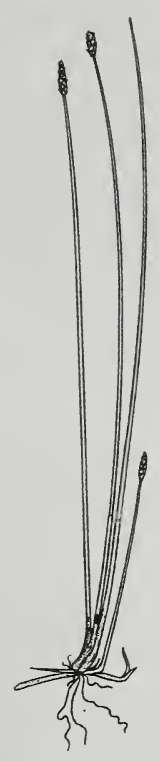

Elymus arenarius

beach rye
ELARM

Gramineae

HABIT: Culms from long, stout, creeping rootstocks.

KEY CHARACTERS: Old leaves at base persistent after withering. Leaves firm, flat, with edges rolled inward. Stiff spike, spikelets 3-7 flowered.

HABITAT: Sandy beaches, forming a belt along shore, rare on dunes inland.

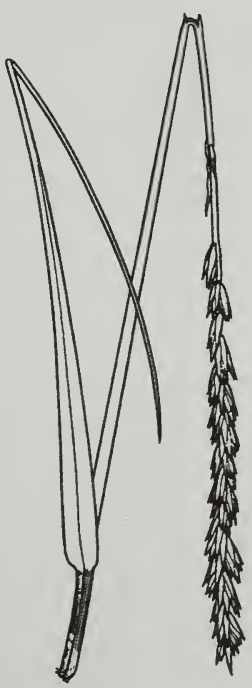




\section{Eriophorum angustifolium ERAN6 tall cottongrass \\ Cyperaceae}

HABIT: Rhizomatous, 4-35 inches.

KEY CHARACTERS: Leaves usually flat below the middle, triangular channeled or folded above the middle. Spikes 2-10, at least some pendulous, scales grayish. Flower heads with long, silky, white bristles.

HABITAT: Muskegs, bogs, meadows, shallow ponds, alpine tundra, heath, open woods.

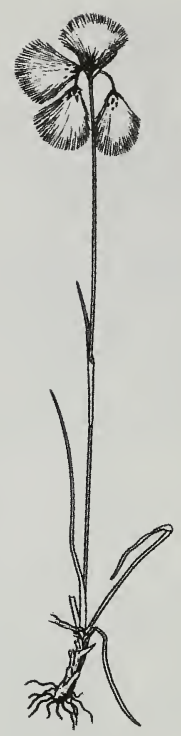

Eriophorum russeolum red cottongrass
ERRU2

Cyperaceae

HABIT: Rhizomatous, 8-30 inches.

KEY CHARACTERS: Leaves linear, channeled. Spikes solitary, erect, more or less round. Flower heads with long, silky, cinnamon to white bristles.

HABITAT: Bogs, lakeshores, muskegs, wet meadows, stream banks, alpine tundra, heath, open woods.

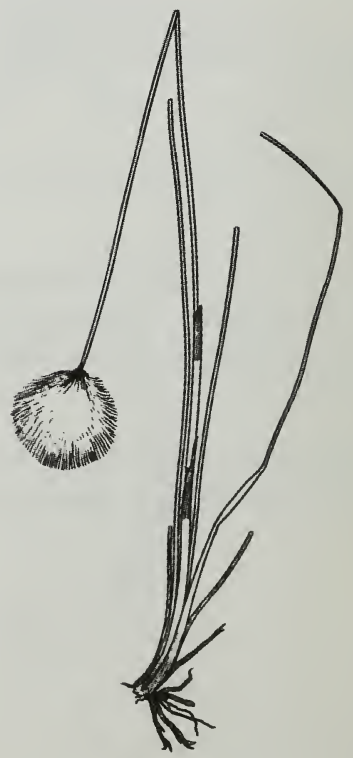


Festuca altaica rough fescue
FEAL

Gramineae

HABIT: Perennial, tufted, 8-32 inches.

KEY CHARACTERS: Leaf blades folded. Inflorescence a 3-6 inch long open panicle. Spikelets with 2-6 flowers. Lemmas purplish to green, with short terminal awn.

HABITAT: Open forests, streambanks, gravel bars, rock outcrops.

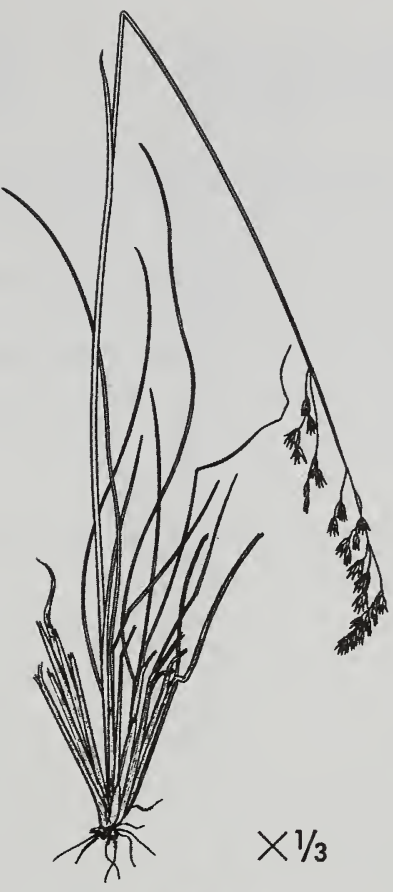

Festuca rubra

FERU2

red fescue

HABIT: Perennial, tufted, to 25 inches.

KEY CHARACTERS: Leaf blades folded or rolled in from edges. Inflorescence a 14 inch long compact or open panicle, often to 1 side of stem. Spikelets purple to green, with 4-7 flowers. Lemmas usually with short terminal awn.

HABITAT: Muskegs, cliffs, beaches, tidal flats.

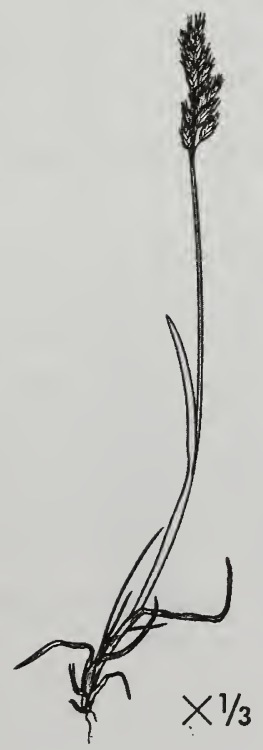


Glyceria pauciflora

false mannagrass

HABIT: Rhizomatous, often rooting at the lower nodes, 10-40 inches tall.

KEY CHARACTERS: Leaf blades flat, to $3 / 5$ inches wide, rough-hairy on one or both sides. Panicle mostly 4-9 inches long, loose, branches ascending to spreading or drooping. Spikelets 3-7 flowered, lemmas with 5-7 prominent nerves.

HABITAT: Woods, thickets, marshes, meadows.

GLPA6

Gramineae

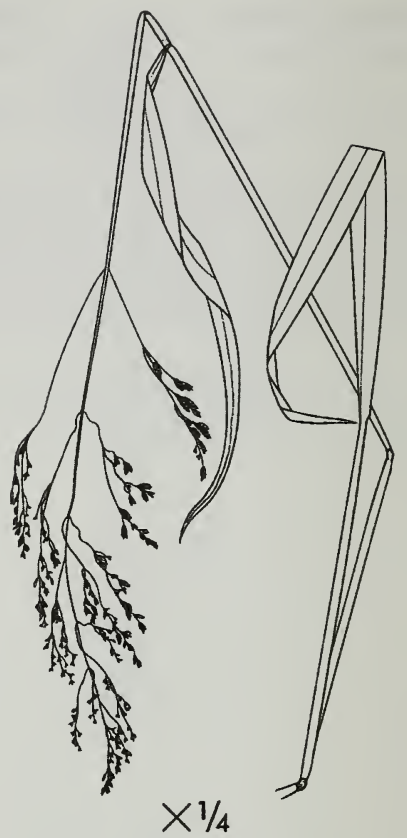

HIAL3

Gramineae
Hierochloë alpina

alpine holygrass

HABIT: Tufted, shortly rhizomatous, $1 / 2-1$ foot.

KEY CHARACTERS: Leaf blades rolled under at edge. Inflorescence a $1-$ to 2 -inch long open panicle. Spikelets with 3 flowers. Stamen-bearing lemmas hairy, the first with short awn, the second with long, twisted and bent awn arising from middle of lemma. Fertile lemmas hairy at tip, awnless. Glumes about as long as spikelet.

HABITAT: Open forests, tundra.

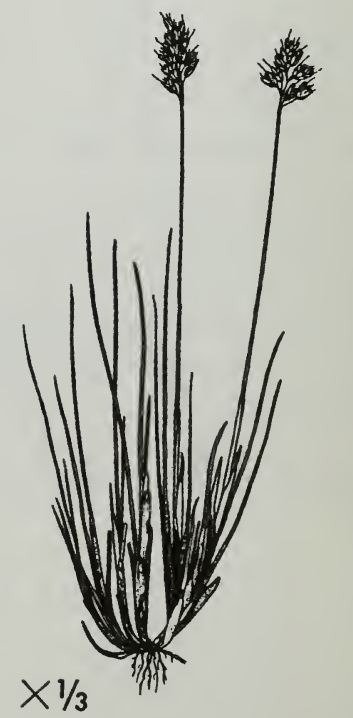




\section{Hordeum brachyantherum HOBR2}

meadow barley

Gramineae

HABIT: Tufted, 6-33 inches tall.

KEY CHARACTERS: Leaf blades flat. Inflorescence an erect spike $1 \frac{1}{2}-5$ inches long, easily shattering when mature. Glumes all slender, awnlike. Central floret usually with an awn surpassing those of the glumes.

HABITAT: Open woods, tidal flats, beaches, bluffs, disturbed areas.

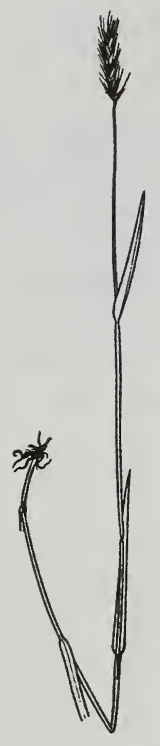

Juncus alpinus

alpine rush
JUAL

Juncaceae

HABIT: Perennial from short rhizomes, culms tufted, 4-18 inches tall.

KEY CHARACTERS: Leaves 1-3 per stem. Leaf blades stiff, erect, round in cross-section. Inflorescence terminal, the branches ascending, heads commonly 5-many, 3-10 flowered.

HABITAT: Sandy banks and shores and moist sites near lakes, streams, and ponds.

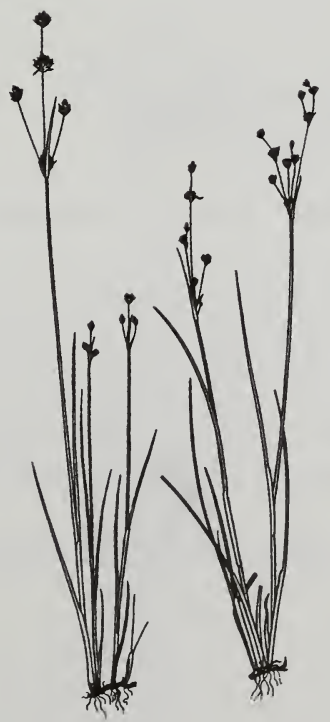


Juncus arcticus arctic rush
JUAR2

Juncaceae

HABIT: Culms stout, thick, with yellowish-brown, shiny basal sheaths in rows from thick, horizontal, dark rootstocks.

KEY CHARACTERS: Contracted flower cluster, many-flowered, outer flower leaves linear-lanceolate, brown with greenish center, longer than inner.

HABITAT: Sandy shores, tidal marshes.

(subsp. sitchensis, subsp. alaskanus and subsp. ater similar, also found in wet places and river flats.)

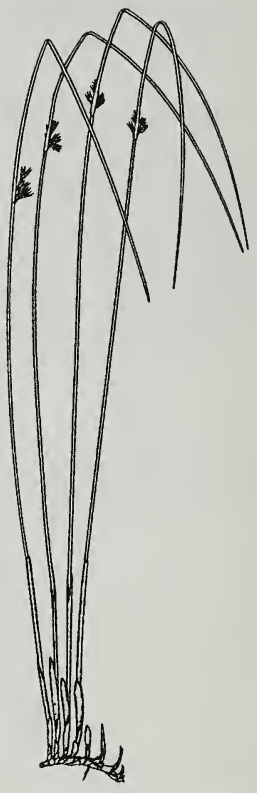

Luzula multiflora common woodrush

LUMU2 Juncaceae

HABIT: Tufted, to 18 inches.

KEY CHARACTERS: Leaves narrow, with closed sheaths. 2-4 stem leaves. Stem solid and round. Flowers crowded in a spike, with 3 petals and 3 sepals, all scale-like. Fruit 1-parted, 3-seeded capsules, longer or shorter than "petals and sepals."

HABITAT: Wet to dry forests and tundra.

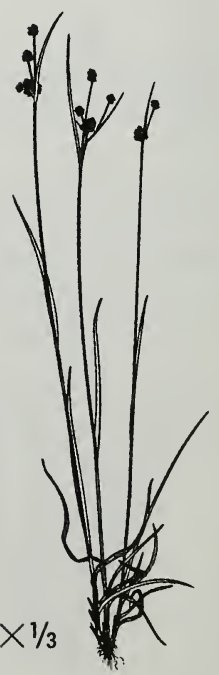


Luzula parviflora smallflowered woodrush
LUPA4

Juncaceae

HABIT: Tufted or solitary from rhizomes, 6-38 inches.

KEY CHARACTERS: Leaves broad, with closed sheaths. 3-5 stem leaves. Stem solid and round. Flowers solitary at tips of branches of open inflorescence, with 3 petals and 3 sepals, all scale-like. Fruit 1-parted, 3-seeded capsules, at least as long as "petals and sepals."

HABITAT: Forests, meadows.

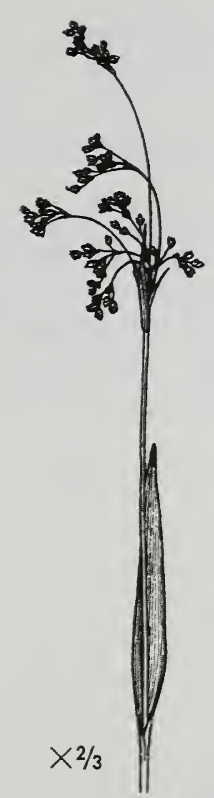

Luzula wahlenbergii

Wahlenberg's woodrush

LUWA

Juncaceae

HABIT: Tufted and shortly rhizomatous, 4-16 inches tall.

KEY CHARACTERS: Leaves blades flat. Inflorescence an open, nodding, or spreading panicle, the flowers solitary or in $2 \mathrm{~s}$ or $3 \mathrm{~s}$ at the end of hairlike branches.

HABITAT: Most sites in alpine tundra, heath, and open woods.

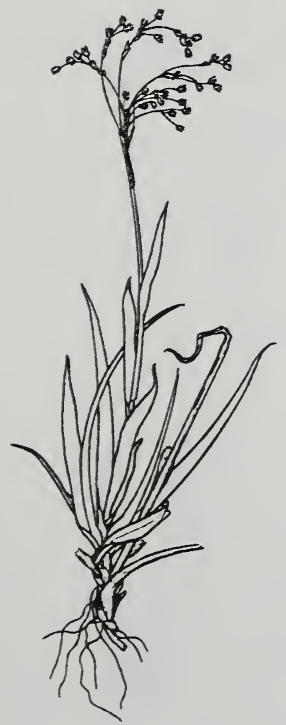


Phleum commutatum mountain timothy
PHCO9

Gramineae

HABIT: Perennial, tufted, 5-26 inches.

KEY CHARACTERS: Inflorescence a 1/2to 2-inch long, short-branched, dense, spike-like, cylindrical panicle. Spikelets with 1 flower. Lemmas finely hairy, awnless. Glumes with hairs on central ridge and sides, with awn up to $3 / 4$ length of glume.

HABITAT: Open forests, meadows, streambanks.

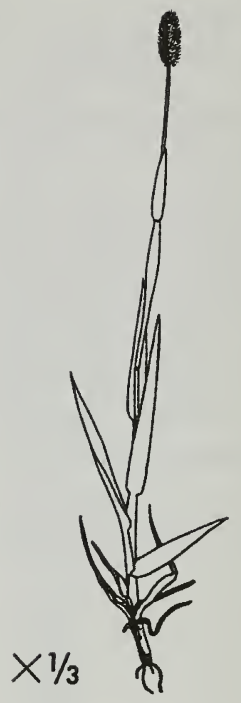

Poa lanata arctic bluegrass
POLA

Gramineae

HABIT: Perennial from creeping rhizomes, 8-30 inches tall.

KEY CHARACTERS: Leaves short, stiff. Panicle pyramid-shaped. Spikelets purplish, 3-6 flowered, lemmas densely hairy with woolly hairs in lower half.

HABITAT: Meadows.

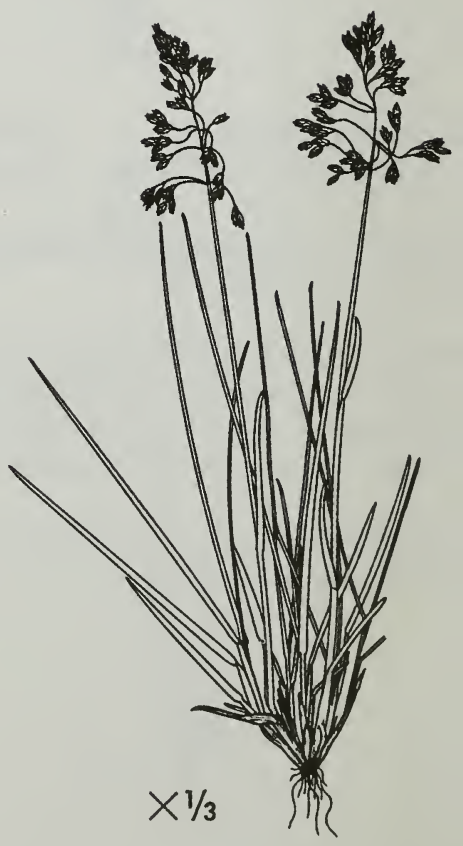


Poa macrantha seashore bluegrass
POMA26

Gramineae

Not in Hultén (1968). See Pojar and Mackinnon (1994).

HABIT: Perennial with extensively creeping rhizomes and long runners, upright, 5-15 inches tall.

KEY CHARACTERS: Leaves are stiff, inrolled, in dense tufts. Panicle compact, $1 \frac{1}{2}-5$ inches long. Spikelets 5-flowered, long ( $1 / 2$ inches) relative to most species of Poa.

HABITAT: Coastal sand dunes.

Puccinellia nutkaensis Nootka alkaligrass
PUNU

Gramineae

HABIT: Tufted perennial, 8-25 inches tall.

KEY CHARACTERS: Leaf blades flat to infolded. Panicles $1 \frac{1}{2}-7 \frac{1}{2}$ inches long, the branches appressed-ascending to spreading. Spikelets mostly 4-6 flowered, green or purplish.

HABITAT: Seashores.

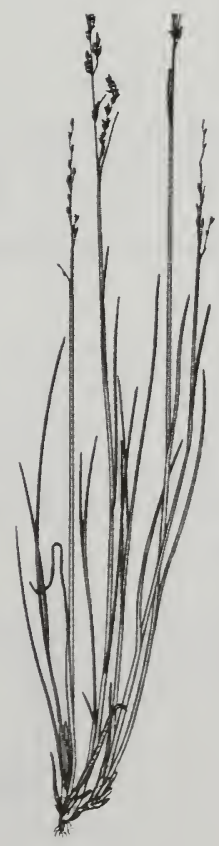


Puccinellia pumila

dwarf alkaligrass
PUPU

Gramineae

HABIT: Tufted perennial, 3-8 inches tall.

KEY CHARACTERS: Leaf blades infolded. Panicles 1-3 inches long, the branches usually appressed. Spikelets mostly 3-6 flowered, green or purplish.

HABITAT: Seashores.
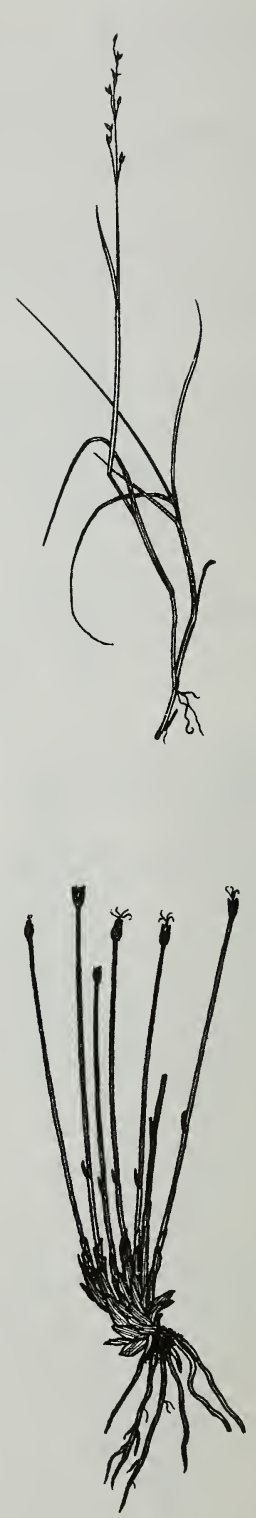

Trichophorum caespitosum TRCA30 tufted bulrush

Cyperaceae

HABIT: Perennial. Densely tufted. Smooth culms.

KEY CHARACTERS: Light green, erect or ascending. Numerous basal sheaths.

Solitary spikelet, terminal, few-flowered and ovoid-oblong. Scales ovate and yellowish-brown, deciduous with 6 smooth bristles.

HABITAT: In acid bogs and on moist rocks in tundra, alpine mats. 
HABIT: Tufted, to 28 inches.

KEY CHARACTERS: Leaves densely hairy. Inflorescence a $1 / 2-$ to 5 inch long open spike-like panicle. Spikelets with 2 or more flowers. Lemmas extend beyond or concealed by glumes, with bent and twisted awn arising from above middle.

HABITAT: Forests, meadows, streambanks, rock outcrops, alpine.

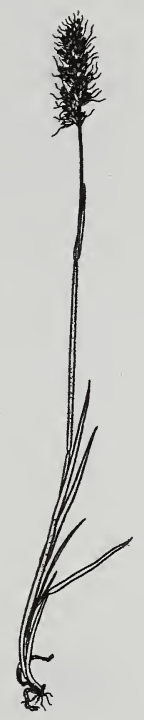

Vahlodea atropurpurea mountain hairgrass

HABIT: Perennial, tufted, to 26 inches.

KEY CHARACTERS: Leaf blades flat and broad. Inflorescence a 1-5 inch long open panicle. Spikelets with 2 or more flowers, green becoming purplish. Florets with hairs $1 / 2$ as long as lemmas. Lemmas concealed by glumes, with twisted awn arising from above middle.

HABITAT: Open forests, meadows.

VAAT2

Gramineae

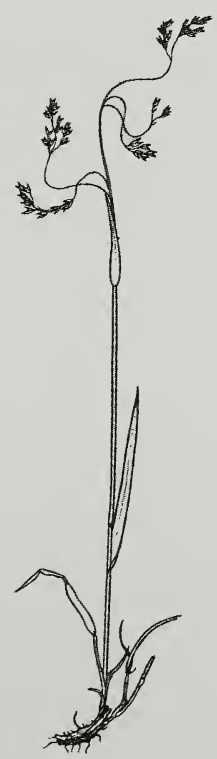




\section{FERNS AND FERN ALLIES}


Athyrium filix-femina

lady fern
ATFI

Athyriaceae

HABIT: Deciduous, tufted, to 2 feet.

KEY CHARACTERS: Blades oval, 2-3 times pinnate. Stalk short, with scales.

HABITAT: Lowlands to 3600 feet. Moist forests, streambanks.

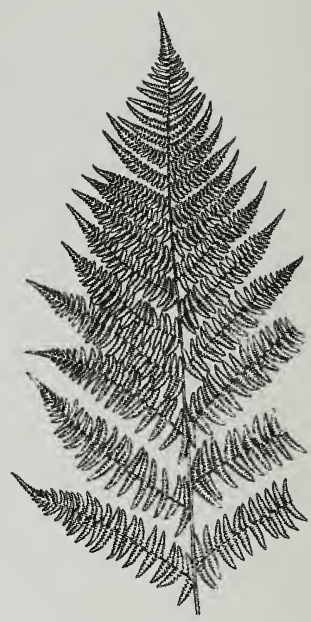

Blechnum spicant

BLSP

deer fern

Blechnaceae

HABIT: Evergreen, tufted, to 2 feet.

KEY CHARACTERS: Sterile blades narrowly oval, evergreen, leathery, with short stalk, forming circle around fertile blades. Fertile blades similar, but taller, deciduous, with narrower "leaflets" and longer stalk. Both types once pinnately divided.

HABITAT: Forests, streambanks, subalpine meadows, acid soil.

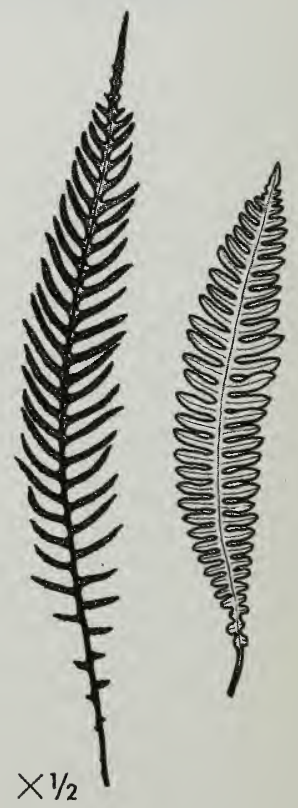


Cystopteris fragilis

fragile fern
CYFR2

Athyriaceae

HABIT: Deciduous, tufted, to 1 foot.

KEY CHARACTERS: Blades 2-3 times pinnate. Stalk shorter than blade.

HABITAT: To 6000 feet. Open forests, talus slopes, rock crevices.

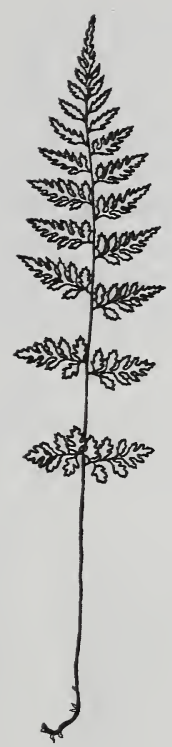

Dryopteris dilatata

DRDI2

wood fern

Aspidiaceae

HABIT: Deciduous, tufted, 1-3 feet.

KEY CHARACTERS: Blade egg-shaped in outline, 3 times pinnate. Lower pair of leaflets triangular. Stalk shorter than blade, with scales.

HABITAT: Forests, streambanks.

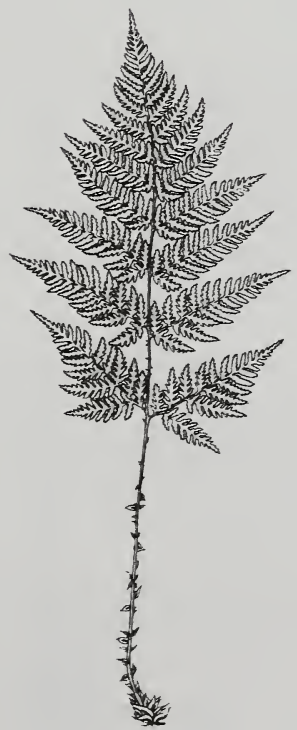


HABIT: Upright, to 30 inches.

KEY CHARACTERS: Stem hollow, jointed, with 10-12 vertical ridges. Branches in whorls. Brown, branchless flowering stems appear in spring, fruiting head withers quickly.

HABITAT: Forests, lakeshores, disturbed areas, beaches, tundra.

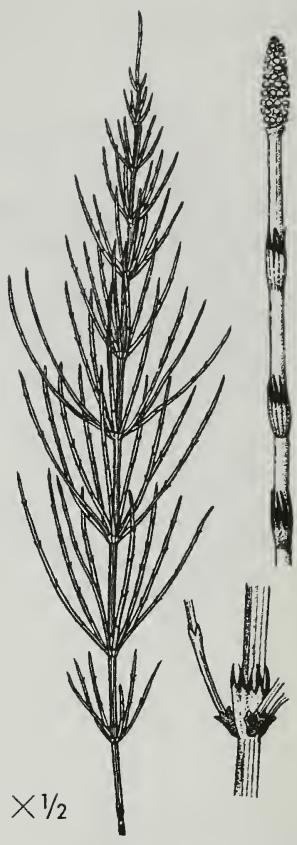

Equisetum fluviatile

EQFL

swamp horsetail

Equisetaceae

HABIT: Rhizome glabrous, simple or branched stem, 10-40 inches tall, with wide central cavity.

KEY CHARACTERS: Stem finely striate, smooth to the touch. Single branches or in irregular whorls at internodes. Green sheaths, with dark brown teeth. Obtuse, long-stalked cones.

HABITAT: Shallow water and marshy places in low altitudes.

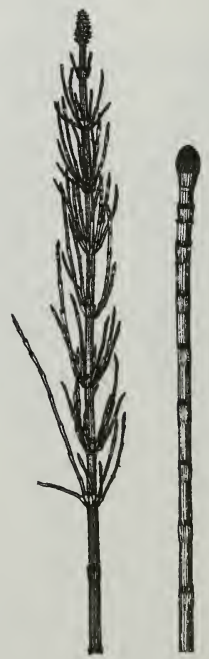


Equisetum palustre marsh horsetail
EQPA

Equisetaceae

HABIT: Thin, smooth rhizome, lustrous, dark reddish-brown, stems 6-24 inches tall.

KEY CHARACTERS: Simple stem with 6 ridges, or with single to several thick branches of different lengths, top of stem usually lacking branches. First sheath of branches very short and dark brown, the next green. Long-stalked cone, obtuse and soon withering.

HABITAT: Wet or moist places, ponds. Rare along shores, more common inland.

EQPR

Equisetaceae
Equisetum pratense meadow horsetail

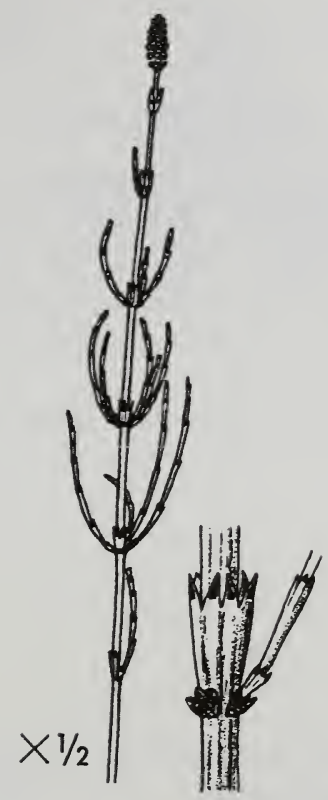

HABIT: Rhizome nearly black, creeping, with scattered stems, 4-20 inches tall.

KEY CHARACTERS: Spring phase with light-brown stem and few short branches, later developing into whorls of branches. Summer phase with grayish-green, thin, striated, very rough stems. Green sheaths. Numerous slender branches, 3-angled. Obtuse, long-stalked cone, soon withering.

HABITAT: Common in woods of the interior.

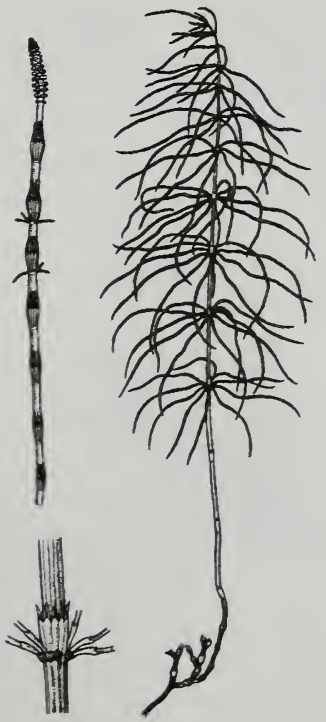


Equisetum sylvaticum woodland horsetail
EQSY

Equisetaceae

HABIT: Upright, to 28 inches.

KEY CHARACTERS: Stem hollow, jointed, with 10-18 vertical ridges. Branches in whorls, branches again branched. Brownish, branched flowering stems appear in spring, fruiting head withers quickly.

HABITAT: To subalpine. Open forests.

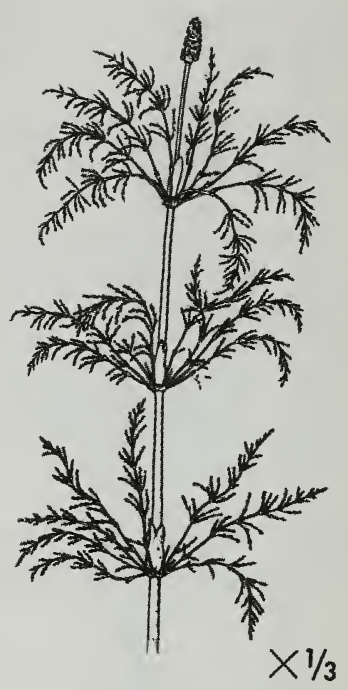

Equisetum variegatum

EQVA

northern horsetail

Equisetaceae

HABIT: Upright, to 15 inches.

KEY CHARACTERS: Stem hollow, jointed, with 5-12 vertical ridges, the central cavity to the diameter of the stem. Fruiting head with a prominent point at tip.

HABITAT: Muskegs, streambanks, woods, alpine tundra.

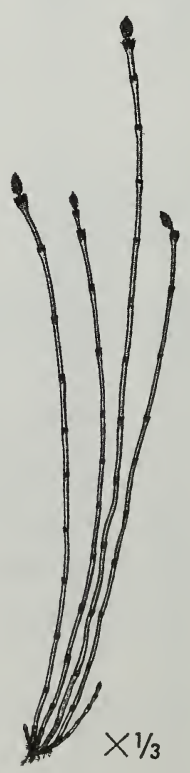


Gymnocarpium dryopteris oak fern
GYDR

Aspidiaceae

HABIT: Deciduous, solitary, 8-12 inches.

KEY CHARACTERS: Blades light green, ternate, the leaflets again 1-2 times pinnate.

HABITAT: Forests, thickets, streambanks, seeps.

\section{Lycopodium alpinum alpine clubmoss \\ LYAL3 \\ Lycopodiaceae}

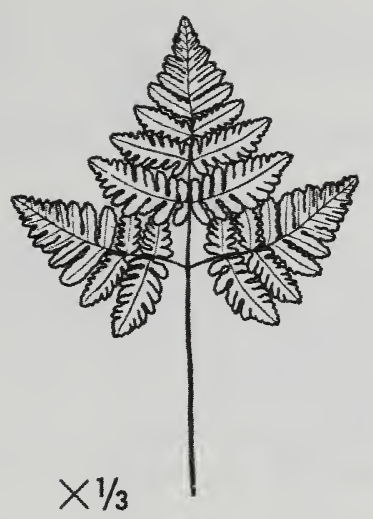

HABIT: Elongate stem creeping in surface of soil, rooting, whitish-green.

KEY CHARACTERS: Erect, blue-green branches, cylindrical or usually slightly flattened, with leaves in 4 rows. Sessile spikes, at tips of leafy branches.

HABITAT: Woods, meadows, and heaths. Common from lowlands to mountains. Absent from calcareous soil.

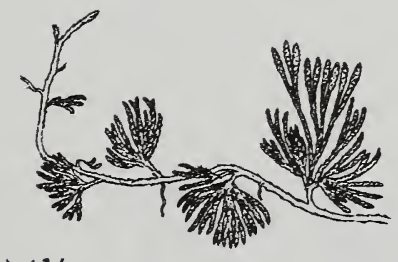

$\times 1 / 3$

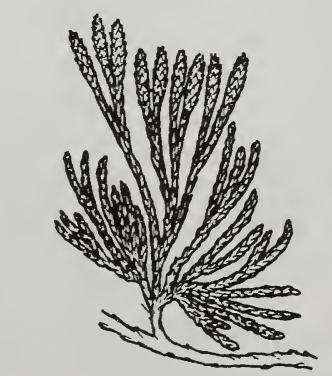

$\times 2 / 3$ 
Lycopodium annotinum stiff clubmoss
LYAN2

Lycopodiaceae

HABIT: Upright from creeping stem, to 8 inches.

KEY CHARACTERS: Leaves in 8 rows along stem, 4 in a whorl, needle-like, toothed, stiff, with pointed tip. 1 terminal stalkless spore-bearing spike per stem.

HABITAT: Lowlands to lower alpine. Forests, heaths, muskegs.

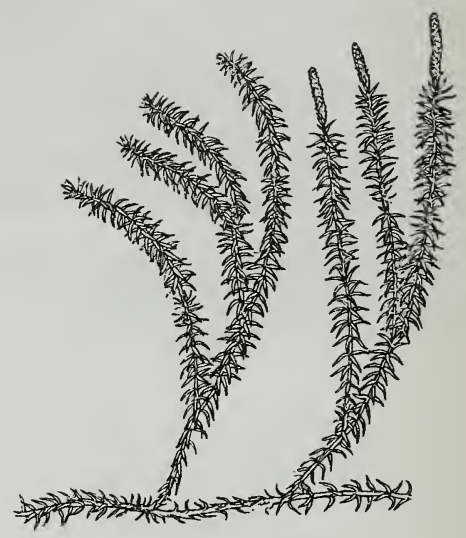

Lycopodium clavatum

LYCL running clubmoss Lycopodiaceae

HABIT: Ascending from creeping stem, to 8 inches.

KEY CHARACTERS: Leaves in 10 rows along stem, needle-like, lying flat on stem, or nearly so, with long hair at tip. 1 terminal fruiting stalk per stem with 1-3 spore-bearing spikes.

HABITAT: To lower alpine. Forests, rocky places, mostly on acid soil.

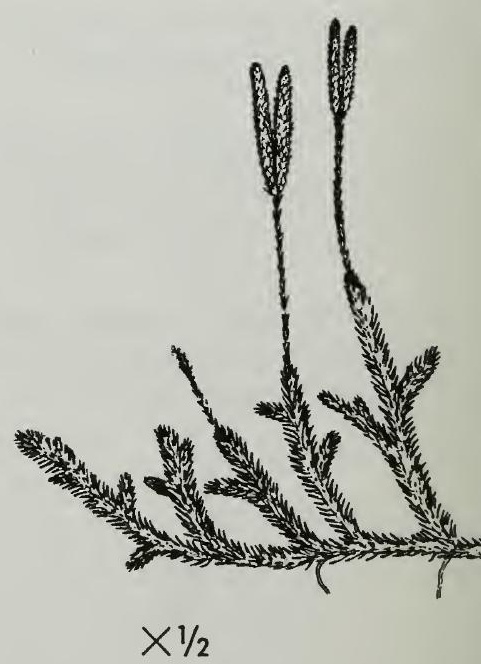


Lycopodium complanatum LYCO3 groundcedar

HABIT: Upright from creeping stem, to 14 inches.

KEY CHARACTERS: Leaves in 4 rows, scale-like, lying flat on stem. Stems flat, with cedar-like appearance. 1 terminal fruiting stalk per branch with 2 spore-bearing spikes.

HABITAT: Rarely above treeline. Dry forests.

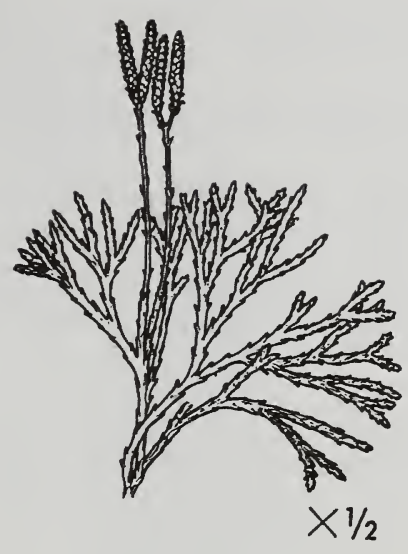

Lycopodium sabinifolium LYSAS3

savinleaf groundpine Lycopodiaceae

HABIT: Ascending, densely branching, stem creeping in surface soil, stems 1-5 inches long.

KEY CHARACTERS: Leaves in 5-6 rows along stem, uniform. Spikes solitary at ends of elongate branches.

HABITAT: Alpine meadows, heath, woods.

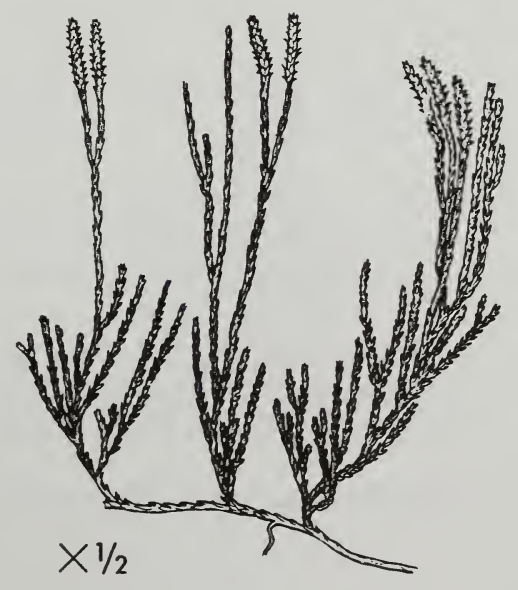




\section{Lycopodium selago \\ fir clubmoss \\ LYSE \\ Lycopodiaceae}

HABIT: Ascending, tufted, 2-5 inches.

KEY CHARACTERS: Leaves in 8 rows along stem, needle-like. Stems branch in pairs, with all branches reaching same height. Spore bearing leaves along stem, not in spikes.

HABITAT: Low elevation to alpine.

Forests, heaths, muskegs, tundra.

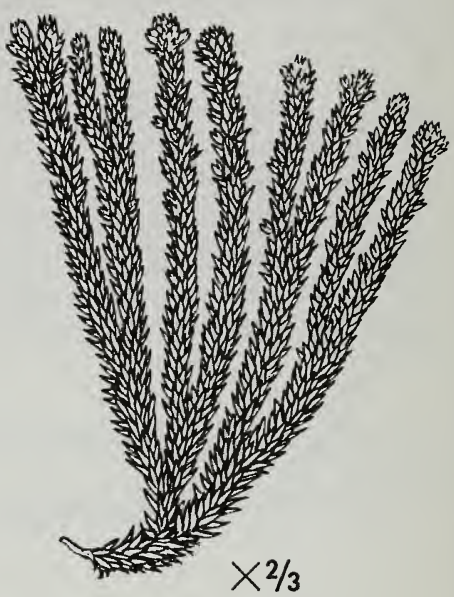

Selaginella selaginoides club spikemoss

SESE

Selaginellaceae

HABIT: Stems of two types, the vegetative ones prostrate and mat-forming, the fertile ones ascending. Stems 1-4 inches long.

KEY CHARACTERS: Leaves spirally arranged, not bristle-tipped. Fruiting structure round in cross section, solitary.

HABITAT: Woods, thickets, heath.

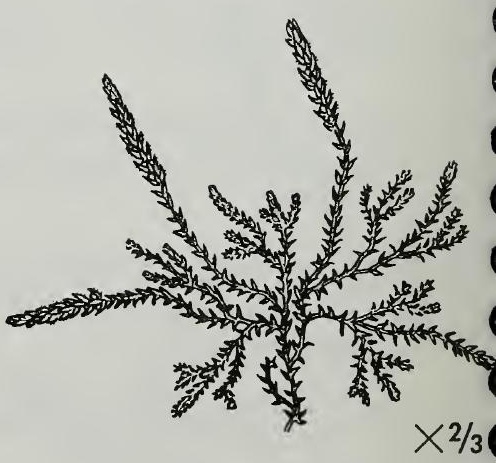


Thelypteris limbosperma maiden fern
THLI9

Thelypteridaceae

HABIT: Short, ascending rhizome, scaly.

KEY CHARACTERS: Leaf stalks grooved above, with dark base. Fronds forming crown, yellowish-green, glandular and covered with white down on veins below, abruptly pointed. Pinnae segments entire, blunt. Sori small, indusium lobed, glandular.

HABITAT: Open, rocky slopes, reaching subalpine region.

\section{THPH}

Thelypteridaceae

Thelypteris
beech fern

HABIT: Deciduous, solitary, to 16 inches.

KEY CHARACTERS: Blade triangular, 1-2 times pinnate, hairy. Stalk hairy, scaly, twice as long as blade. Lower most leaflets droop.

HABITAT: Lowlands to lower alpine. Forests, meadows, streambanks, stony slopes.
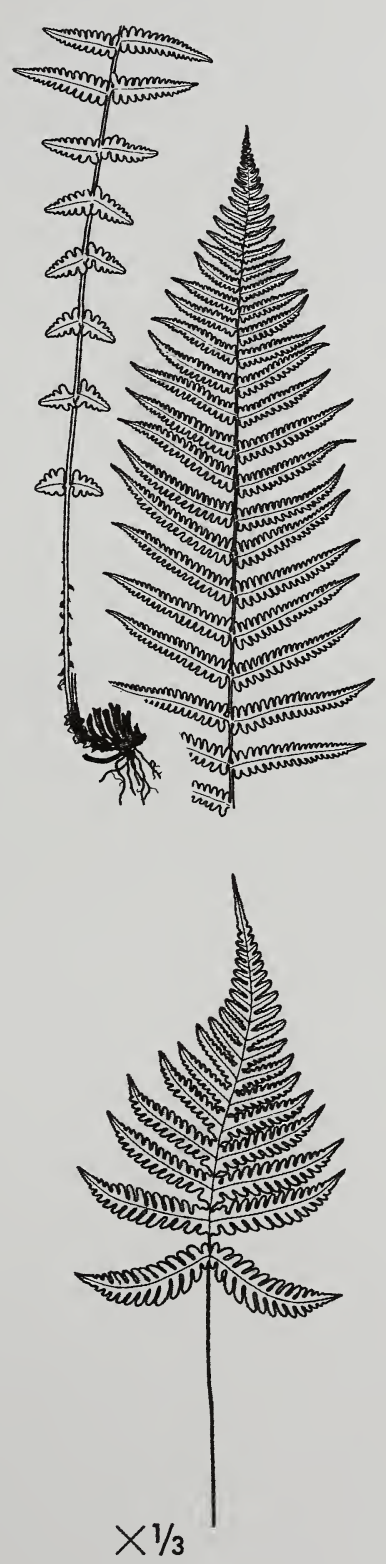


\section{APPENDICES}




\section{Appendix 1. \\ Comparison between Hultén and PLANTS nomenclature.}

Hultén Name

Trees

Betula kenaica

Betula papyrifera

\section{Shrubs}

Alnus crispa ssp. sinuata

Cassiope stelleriana

Cladothamnus pyrolaeflorus

Echinopanax horridum

Ledum palustre

ssp. groenlandicum

Oxycoccus microcarpus

Potentilla fruticosa

Salix arctica

Salix depressa ssp. rostrata

Spiraea beauverdiana

Vaccinium alaskensis

Forbs

Achillea borealis

Apargidium boreale

Aruncus sylvester

Callitriche verna

Cardamine umbellata

Cicuta mackenzieana

Conioselinum chinense

Epilobium adenocaulon

Epilobium angustifolium

Epilobium glandulosum

Epilobium latifolium

Fauria crista-galli

Heracleum lanatum

Lathyrus maritimus

Myosotis alpestris

Nuphar polysepala

Petasites hyperboreus
Code

PLANTS revised name

Code

BEKE2 Betula papyrifera var. kenaica

BEPA Betula neoalaskana

BEPAK

BENE4

ALVIS

ALCRS Alnus viridis ssp. sinuata

HAST3

CAST33 Harrimanella stelleriana

ELPY

CLPY3 Elliottia pyroliflorus

ECHO2 Oplopanax horridus

$\mathrm{OPHO}$

LEPAG Ledum groenlandicum

LEGR

OXMI3 Vaccinium oxycoccos

VAOX

POFR4 Dasiphora floribunda

DAFL3

SAAR4 Salix arctophila

SAAR6

SADER Salix bebbiana

SABE2

SPST3

VAOV
ACMIB

MIBO

APBO Microseris borealis

ARSYA Aruncus dioicus var. acuminatus

ARDIA

CAVE2

Callitriche palustris

CAUM3

Cardamine oligosperma var. kamtschatica

CIMA Cicuta virosa

COCHP Conioselinum gmelinii

EPAD Epilobium ciliatum ssp. ciliatum

EPAN2 Chamerion angustifolium ssp. angustifolium

EPGL4 Epilobium ciliatum ssp. glandulosum EPCIG

EPLA Chamerion latifolium

CHLA13

FACR Nephrophyllidium crista-galli

NECR2

HELA4 Heracleum maximum

HEMA80

LAMA3 Lathyrus japonicus var. maritimus

LAJAM

MYAL Myosotis asiatica

MYAL

Nuphar lutea ssp. polysepala

PEHY5 Petasites frigidus var. nivalis
NULUP

PEFRN 
Platanthera saccata

Potamogeton filiformis

Potamogeton pectinatus

Potamogeton perfoliatus

Potentilla egedii

Potentilla palustris

Pyrola secunda

Rumex fenestratus

Sanguisorba stipulata

Saxifraga punctata

Sedum rosea

Sparganium minimum

Stellaria sitchana

Utricularia vulgaris

\section{Graminoids}

Agrostis alaskana

Carex sitchensis

Elymus arenarius

Glyceria pauciflora

Juncus alpinus

Phleum commutatum

Poa lanata

Puccinellia pumila

\section{Ferns and Fern Allies}

Dryopteris dilatata

Lycopodium sabinifolium

Lycopodium selago

Thelypteris limbosperma

Thelypteris phegopteris
PLSA6 Platanthera stricta

POFI2 Stuckenia filiformis ssp. filiformis

POPE6 Stuckenia pectinatus

POPER5 Potamogeton richardsonii

POEG Argentina egedii ssp. egedii

POPA14 Comarum palustre

PYSE Orthilia secunda

RUFE3 Rumex aquaticus var. fenestratus

SAST11 Sanguisorba canadensis

SAPU6 Saxifraga nelsoniana

ssp. nelsoniana

SEROI3 Rhodiola integrifolia

ssp. integrifolia

SPMI Sparganium natans

STSI3 Stellaria borealis ssp. sitchana

UTVU Utricularia macrorhiza
PLST4

STFIF

STPE12

PORI2

AREGE

COPA28

ORSE

RUAQF

SACA14

SANEN

RHINI

SPNA

STBOS

UTMA
AGAL2 Agrostis exarata

CASI3 Carex aquatilis var. dives

ELARM Leymus mollis ssp. mollis

GLPA6 Torreyochloa pallida var. pauciflora

JUAL Juncus alpinoarticulatus ssp. nodulosus

PHCO9 Phleum alpinum

POLA Poa arctica ssp. lanata

PUPU Puccinellia tenella ssp. alaskana
AGEX

CAAQD

LEMOM2

TOPAP3

JUALN

PHAL2

POARLA

PUTEA

DREX2

LYSI

HUSES

THQU2

PHCO24 

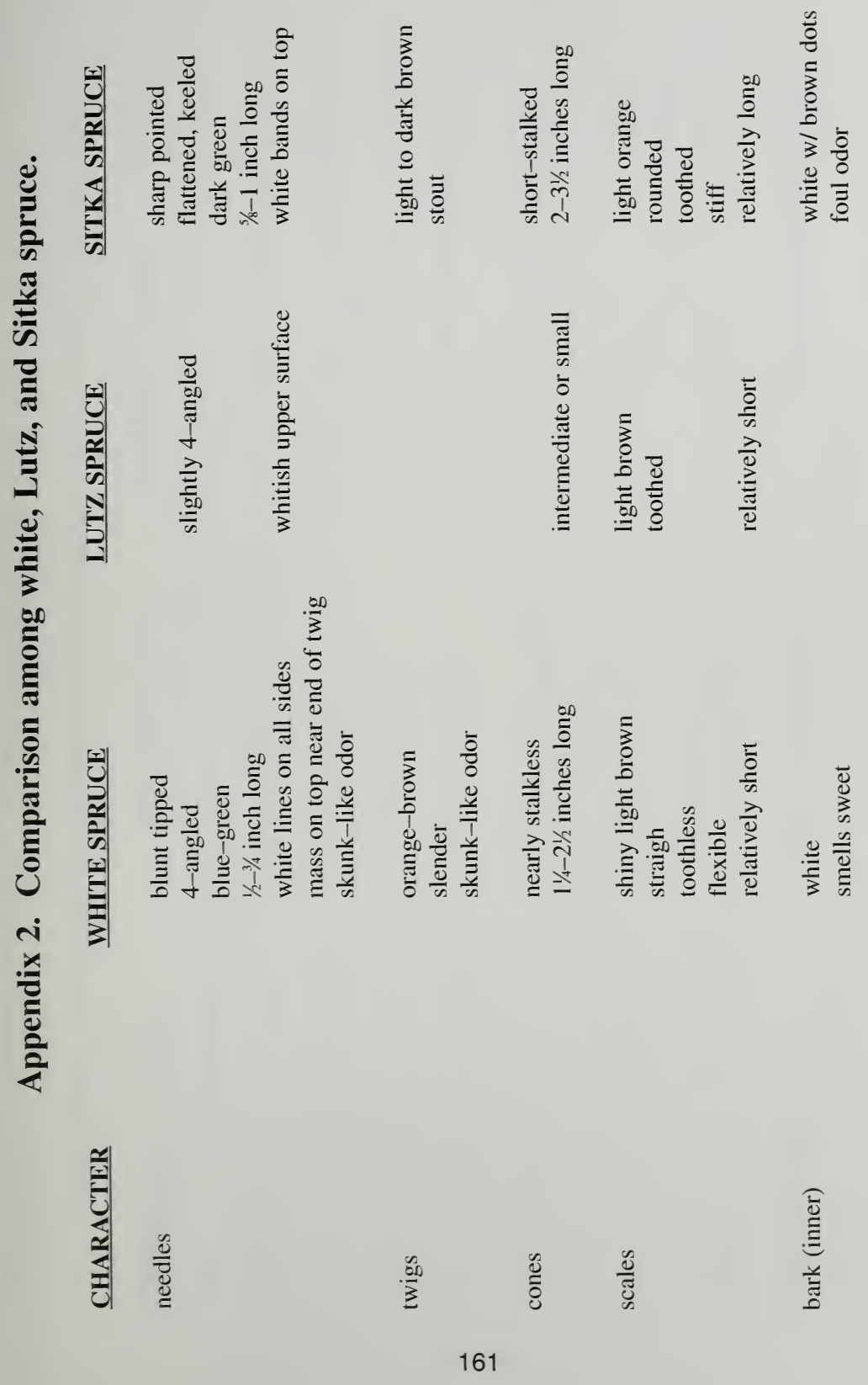


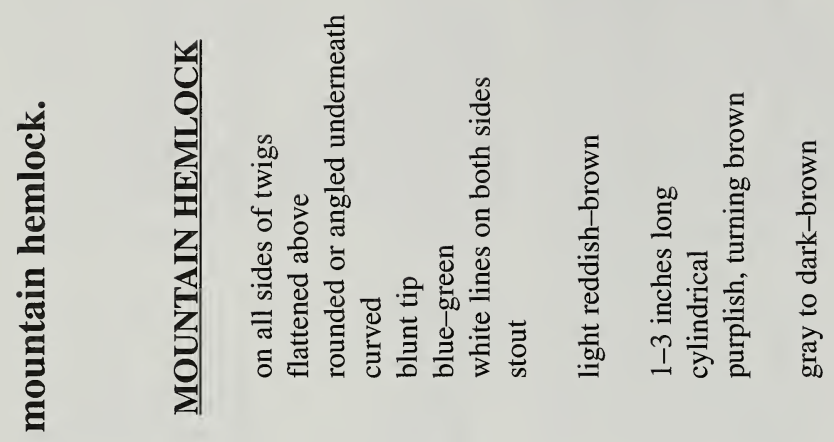

를

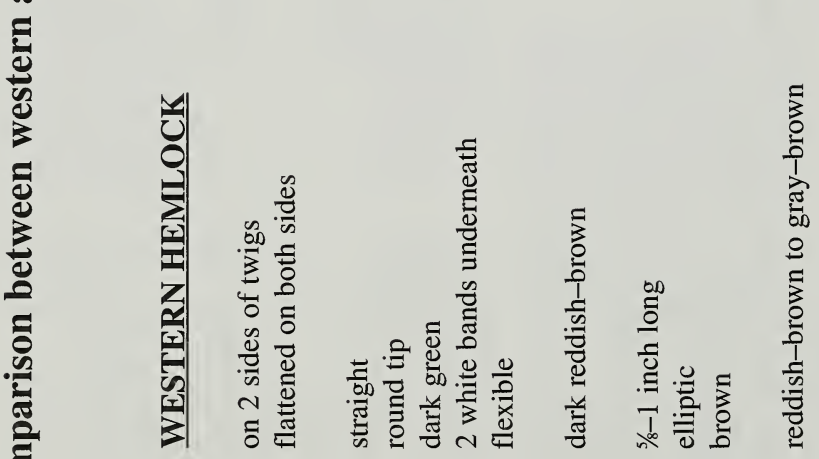

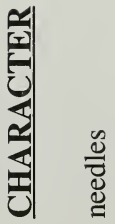

紫

善 
咅

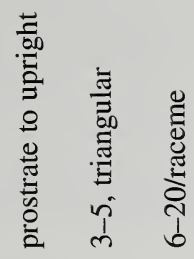

$\stackrel{\mathscr{d}}{\circlearrowright}$

言

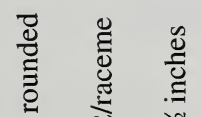

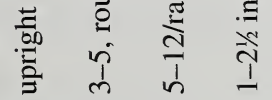

+ 远

를 를

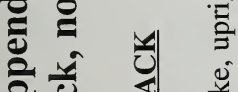

$\sum \frac{0}{2} \stackrel{0}{2}$

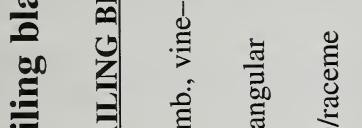

Е

है

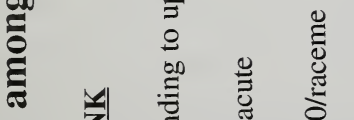

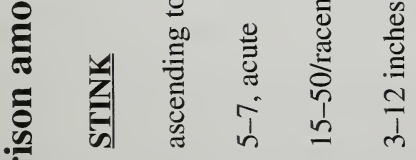

政

है

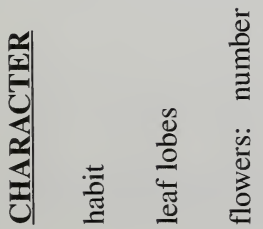

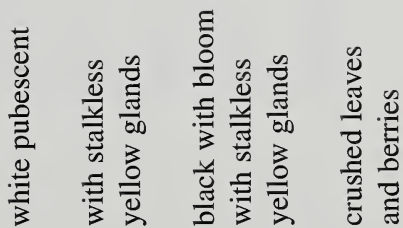

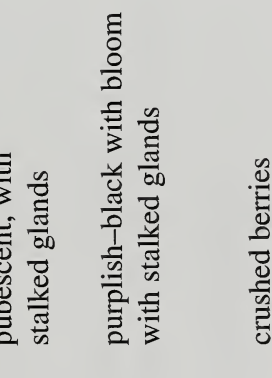

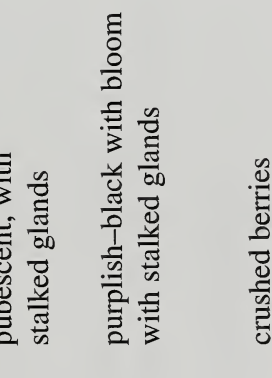

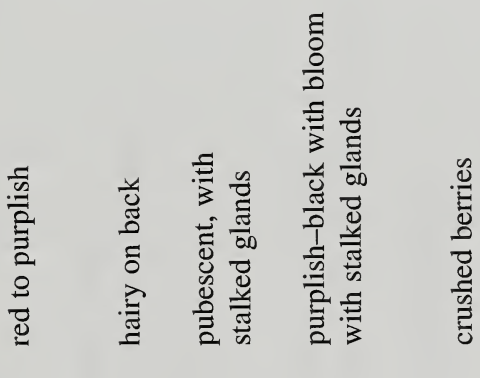

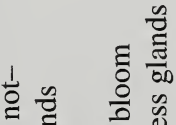

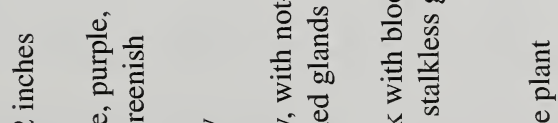

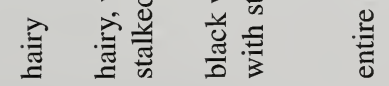

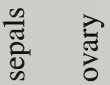

. 

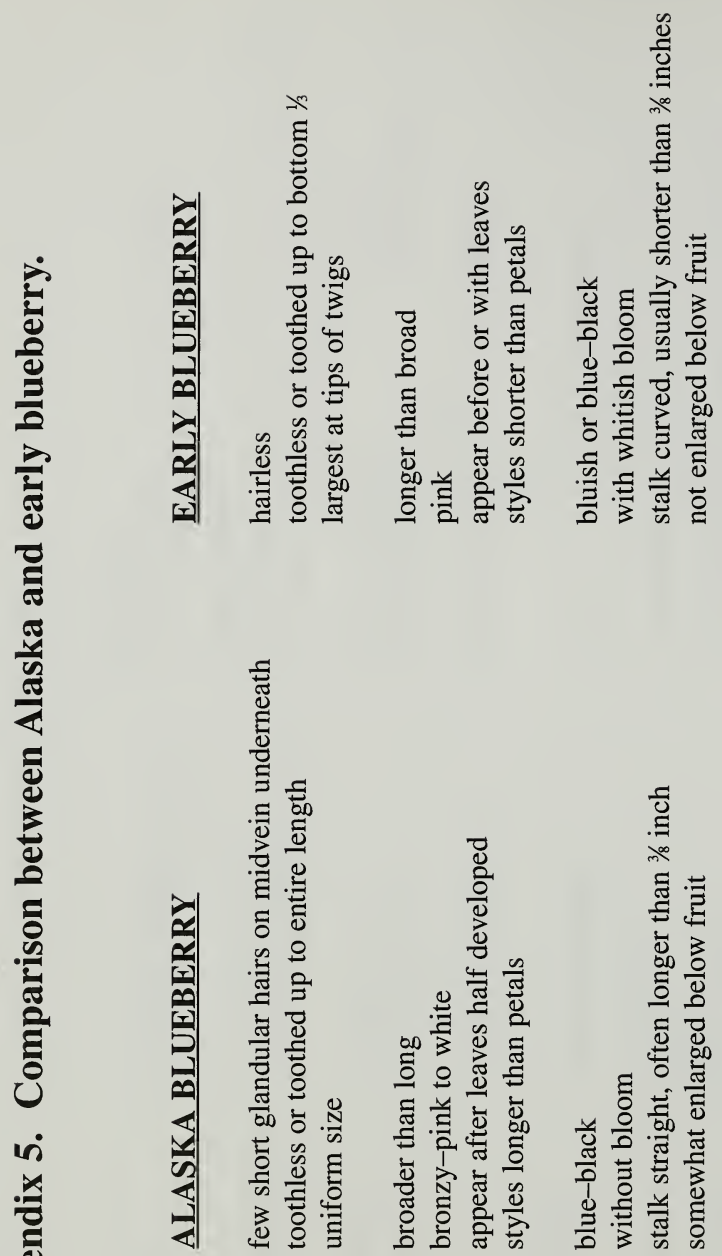

돈)

这 


\section{Appendix 6. Glossary.}

ACHENE - a small, dry, hard, one-seeded fruit.

ALTERNATE - describes the arrangement of leaves on a stem, one per node, with leaves alternating on either side of the stem.

ANTHER - the portion of the stamen that contains the pollen.

ASCENDING - curving upward.

AWN - slender, generally terminal bristle.

AXIL - the upper angle formed between the axis and an organ that arises from it.

BASAL - at or pertaining to the base.

BEAK - a prominent elongate tip.

BIENNIAL - living for two growing seasons.

BLADE - the expanded part of a leaf.

BLOOM - a whitish, waxy, powdery covering.

BRACT - a small leaf from the base of which a flower or a floral axis arises; also a small leaf just below the flower or flower cluster.

BRISTLE - a stiff hair.

CAESPITOSE - growing in dense, low tufts.

CAPSULE - a dry fruit that splits open at maturity. Made of more than one carpel.

CARYOPSIS - in grasses, an achene-like fruit in which the pericarp is fused to the seed.

CATKIN - a scaly spike bearing apetalous, unisexual flower.

COMPOUND LEAF - a leaf divided into two or more parts or leaflets.

COROLLA - the petals of a flower, collectively.

CULM - the type of hollow or pithy slender stem found in grasses or sedges.

CYME - a flat topped or convex flower cluster, with central flowers opening first. 
DECIDUOUS - falling after completion of the normal function; not persistant.

DECUMBENT - lying flat but tending to curve upward at the end.

DIVISION - segments of a leaf separated by a notch cut almost but not all the way to the base or midvein.

DRUPE - a fleshy one-seeded fruit with the seed enclosed in a hard shell, such as a plum.

ENTIRE - pertaining to margins, not toothed or otherwise cut.

EVERGREEN - remaining green throughout the year, not deciduous.

EXCURRENT - projecting beyond the edge of.

FLORET - in grasses, the flower parts with the subtending bracts (lemma and palea).

FOLLICLE - a dry fruit that splits open along one seam.

FROND - a leaf, especially of a fern.

GLABROUS - smooth, devoid of pubescence or hair in any form.

GLAND - a secreting surface or structure; an appendage having the general appearance of such an organ.

GLUME - one of the two bracts found at the base of a grass spikelet.

HEAD - a dense spherical or flat-topped inflorescence of stalkless flowers clustered on a common receptacle.

HIP - the fleshy fruit of the rose.

HYBRID - a plant whose parent plants are two different species or subspecies.

INFLORESCENCE - a cluster of flowers.

KEEL - a central dorsal ridge.

LEAFLET - a single division of a compound leaf.

LEMMA - the outer of the two bracts subtending the individual flowers in grass spikelets.

LIGULE - the showy part of the outer ring of flowers of the Composite Family 
(Compositae).

LOBED - bearing lobes; loosely used, but usually cut in not over halfway to the base or midvein.

LYRE-SHAPED - pinnatifid, with the terminal lobe considerably larger thatn the others.

NEEDLE - a modified leaf that is long, slender, rather rigid and more or less sharp at the apex.

NODE - the place on the stem where leaves or branches normally originate.

OPPOSITE - situated directly across from each other at the same node.

OVARY - the seed-bearing part of the flower.

PALEA - the inner of the two bracts subtending the individual flowers in grass spikelets.

PALMATE - hand shaped, resembling the open, spread hand. The lobes or divisions attached at the base.

PANICLE - a branched inflorescence with the youngest flowers borne near the tip.

PEDUNCLE - a flower-stalk supporting a cluster of flowers, or a single flower when the pedicle is very long.

PERENNIAL - living for more than two years.

PERIANTH - the floral envelope consisting of sepals and petals.

PERICARP - the wall of the fruit, or seed-vessel.

PERIGYNIA - the pistil and surrounding bract of sedges.

PERIGYNIUM - the inflated saclike organ, or utricle, surrounding the pistil in carex.

PETALS - usually, the colorful part of the flower above the sepals.

PETIOLE - the leaf stalk.

PINNATE - leaflets arranged along each side of a common stem of a compound leaf.

PISTIL - the seed-bearing organ of a flower, consisting when complete of an ovary, style, and stigma. 
PRICKLE - a small, sharp, slender growth.

PROSTRATE - lying flat on the ground.

RACEME - an inflorescence with a central stem and stalked flowers, the youngest flowers at the tip.

RACHIS - axis of a compound leaf, or a spike or raceme.

RHIZOME - a prostrate elongated underground stem.

ROSETTE - a cluster of spreading or radiating basal leaves.

SCABROUS - rough to the touch.

SACCATE - bag-shaped; pouchy

SCALE - any thin, dry, membranaceous structure.

SEPAL - one of the parts of the outer whorl of the flower, usually resembling a small green leaf, but may be similar in appearance to the petals.

SESSILE - without a stalk.

SHEATH - the part of the leaf that envelops the stem.

SILIQUE - a narrow, many-seeded capsule, usually more than 4 times as long as broad.

SOLITARY - one per stalk.

SPIKE - an inflorescence with a central stem and stalkless flowers.

SPIKELET - in grasses, the basic unit of the inflorescence in which the floret(s) are found at least partially covered by the two basal bracts (glumes).

SPINE - a sharp woody or rigid growth.

SPORANGIA - a structure within which spores are produced.

SPREADING - with nearly prostrate stems or branches.

SPUR - a tubular or sac-like projection from a blossom as of a petal or sepal; it usually contains a nectar-secreting gland.

STAMEN - the pollen-bearing organ of the flower. 
STOLON - a trailing shoot above ground, rooting at the nodes, also called a runner.

STYLE - a prolongation of the ovary commonly bearing the stigma.

TERMINAL - arising from the end of the stem.

TERNATE - with 3 leaflets.

UMBEL - an inflorescence, more or less flattopped, in which all of the pedicels arise from the same point, like the ribs of an umbrella.

WHORL - an arrangement of three or more leaves at one node on the stem. 


\section{Appendix 7. Common names index}

\section{TREES}

Alaska yellow cedar

balsam poplar

black cottonwood

black spruce

Kenai birch

Lutz spruce

mountain hemlock

paper birch

quaking aspen

Scouler willow

Sitka spruce

western hemlock

white spruce

\section{SHRUBS}

Alaska blueberry

Aleutian mountain heath

alpine azalea

alpine bearberry

arctic willow

Barclay willow

Beauverd spiraea

Bebb willow

bog blueberry

bog cranberry

bog rosemary

bristly black currant

buffaloberry

common juniper

copperbush

creeping sibbaldia

crowberry

devil's club

diapensia

dwarf birches

dwarf blueberry

early blueberry

elderberry

feltleaf willow
Chamaecyparis nootkatensis

Populus balsamifera subsp. balsamifera

Populus balsamifera subsp. trichocarpa 8

Picea mariana

Betula kenaica

Picea X lutzii

Tsuga mertensiana

Betula papyrifera

Populus tremuloides

Salix scouleriana

Picea sitchensis

Tsuga heterophylla

Picea glauca

Vaccinium alaskensis $\quad 40$

Phyllodoce aleutica 26

Loiseleuria procumbens $\quad 23$

Arctostaphylos alpina $\quad 15$

Salix arctica $\quad 32$

Salix barclayi $\quad 32$

Spiraea beauverdiana 39

Salix depressa subsp. rostrata 33

Vaccinium uliginosum $\quad 41$

Oxycoccus microcarpus $\quad 25$

Andromeda polifolia $\quad 14$

Ribes lacustre $\quad 28$

Shepherdia canadensis $\quad 37$

Juniperus communis 21

Cladothamnus pyrolaeflorus $\quad 18$

Sibbaldia procumbens $\quad 38$

Empetrum nigrum 20

Echinopanax horridum 20

Diapensia lapponica 19

Betula glandulosa/nana $\quad 16$

Vaccinium caespitosum $\quad 40$

Vaccinium ovalifolium 41

Sambucus racemosa 37

Salix alaxensis 31 
four-angled cassiope

Greenland Labrador-tea

highbush cranberry

Hooker willow

kinnikinnick

least willow

low blueberry willow

lowbush cranberry

luetkea

Mertens cassiope

narrow-leaf Labrador-tea

netleaf willow

northern black currant

northern red currant

Oregon crab apple

prickly rose

raspberry

red bearberry

rusty menziesia

salmonberry

serviceberry

shrubby cinquefoil

Sitka alder

Sitka mountain-ash

Sitka willow

skunk currant

Steller's cassiope

stink currant

sweetgale

tealeaf willow

thinleaf alder

trailing black currant

twinflower

undergreen willow

western mountain-ash

white mountain-avens
Cassiope tetragona

Ledum palustre subsp. groenlandicum

22

Viburnum edule

Salix hookeriana

34

Arctostaphylos uva-ursi $\quad 16$

Salix rotundifolia $\quad 36$

Salix myrtillifolia $\quad 34$

Vaccinium vitis-idaea $\quad 42$

Luetkea pectinata 23

Cassiope mertensiana 17

Ledum palustre subsp. decumbens 21

Salix reticulata 35

Ribes hudsonianum 28

Ribes triste $\quad 29$

Malus fusca 24

Rosa acicularis $\quad 30$

Rubus idaeus $\quad 30$

Arctostaphylos rubra $\quad 15$

Menziesia ferruginea $\quad 24$

Rubus spectabilis $\quad 31$

Amelanchier spp. $\quad 14$

Potentilla fruticosa $\quad 26$

Alnus crispa subsp. sinuata $\quad 13$

Sorbus sitchensis $\quad 39$

Salix sitchensis $\quad 36$

Ribes glandulosum 27

Cassiope stelleriana $\quad 17$

Ribes bracteosum 27

Myrica gale 25

Salix pulchra $\quad 35$

Alnus incana subsp. tenuifolia $\quad 13$

Ribes laxiflorum 29

Linnaea borealis 22

Salix commutata 33

Sorbus scopulina 38

Dryas octopetala 19 
Alaska Indian paintbrush

Alaska violet

Aleutian mugwort

alpine bistort

alpine heuchera

alpine sweetvetch

alpine willow herb

apargidium

arctic stitchwort

arctic sweet coltsfoot

arrowleaf groundsel

awlwort

baneberry

beach pea

beach lovage

beach strawberry

bent-leaved angelica

blackish oxytrope

bluebells of Scotland

blunt-fruit sweet-cicely

boreal bog orchid

boreal sagebrush

broadleaf arnica

buckbean

bunchberry

calthaleaf avens

capitate lousewort

chiming bells

chocolate lily

claspingleaf pondweed

cloudberry

common alplily

common bladderwort

common butterwort

common marestail

cow parsnip

curled starwort

deerberry

deer cabbage

dwarf fireweed

enchanter's nightshade
Castilleja unalaschcensis

Viola langsdorffii

120

Artemisia tilesii

Polygonum viviparum

94

Heuchera glabra

74

Hedysarum alpinum

73

Epilobium anagalladifolium

62

Apargidium boreale

Minuartia arctica

83

Petasites hyperboreus

Senecio triangularis

108

Subularia aquatica

113

Actaea rubra

Lathyrus maritimus

Ligusticum scoticum

Fragaria chiloensis

Angelica genuflexa

Oxytropis nigrescens

Campanula rotundifolia

Osmorhiza depauperata

Platanthera dilitata

Artemisia arctica

Arnica latifolia

Menyanthes trifoliata

Cornus canadensis

Geum calthifolium

Pedicularis capitata

Mertensia paniculata

Fritillaria camschatcensis

Potamogeton perfoliatus

Rubus chamaemorus

Lloydia serotina

Utricularia vulgaris

Pinguicula vulgaris

Hippuris vulgaris

Heracleum lanatum

Stellaria crispa

Maianthemum dilatatum $\quad 82$

Fauria crista-galli

Epilobium latifolium

Circaea alpina 
false hellebore

fern leaf goldenthread

fewflower meadowrue

fiveleaf bramble

fleshy starwort

floating burreed

floating pondweed

foamflower

forget-me-not

fourleaf marestail

fragrant bedstraw

fringed willow herb

goatsbeard

goosetongue plantain

greenish wintergreen

ground-cone

grove sandwort

heart-leaf twayblade

heart-leaved saxifrage

hooded ladies' tresses

Hornemann's willow herb

Labrador lousewort

largeflowered wintergreen

large-leaf avens

Langsdorf's lousewort

larkspur

little western bittercress

liverleaf wintergreen

lyreleaved rockcress

longbract frog orchid

Mackenzie's water hemlock

marsh fivefinger

marsh violet

monkshood

mountain harebell

nagoonberry

narcissus anemone

Nootka lupine

northern asphodel

northern bedstraw

northern burreed

northern comandra

northern geranium
Veratrum viride

Coptis aspleniifolia

Thalictrum sparsiflorum $\quad 114$

Rubus pedatus

104

Stellaria crassifolia

111

Sparganium angustifolium $\quad 109$

Potamogeton natans 95

Tiarella trifoliata $\quad 115$

Myosotis alpestris $\quad 85$

Hippuris tetraphylla $\quad 75$

Galium triflorum $\quad 69$

Epilobium glandulosum $\quad 63$

Aruncus sylvester $\quad 52$

Plantago maritima 92

Pyrola chlorantha 99

Boschniakia rossica 52

Moehringia lateriflora $\quad 84$

Listera cordata $\quad 79$

Saxifraga punctata $\quad 106$

Spiranthes romanzoffiana $\quad 110$

Epilobium hornemannii $\quad 64$

Pedicularis labradorica $\quad 89$

Pyrola grandiflora $\quad 100$

Geum macrophyllum 72

Pedicularis langsdorfii $\quad 90$

Delphinium glaucum 61

Cardamine umbellata $\quad 55$

Pyrola asarifolia $\quad 99$

Arabis lyrata $\quad 50$

Coeloglossum viride $\quad 58$

Cicuta mackenzieana $\quad 57$

Potentilla palustris $\quad 97$

Viola epipsila $\quad 119$

Aconitum delphinifolium 45

Campanula lasiocarpa $\quad 54$

Rubus arcticus $\quad 103$

Anemone narcissiflora $\quad 46$

Lupinus nootkatensis $\quad 80$

Tofieldia coccinea $\quad 115$

Galium boreale $\quad 68$

Sparganium hyperboreum $\quad 109$

Geocaulon lividum 71

Geranium erianthum 71 
northern goldenrod

northern grass-of-parnassas

northern starwort

northern twayblade

northern waterstarwort

northern willow herb

one-sided wintergreen

Pacific silverweed

pale gentian

pretty shooting star

prickly saxifrage

pygmy pussytoes

rattlesnake root

roseroot stonecrop

round-leaf sundew

sago pondweed

seacoast angelica

seaside arrowgrass

seaside buttercup

seaside sandplant

shortspike watermilfoil

single delight

Sitka burnet

Sitka starwort

Sitka sweet-cicely

Sitka valerian

skunk cabbage

slender bog orchid

slenderfruit willow herb

slender-leaved pondweed

small burreed

smallflower lousewort

snowline wintergreen

spring waterstarwort

spotted gentian

starflower

star gentian

sticky false-asphodel

subalpine fleabane

swamp gentian

swamp willow herb

tall Jacob's-ladder

tall fireweed
Solidago multiradiata

Parnassia palustris

Stellaria calycantha

Listera borealis

Callitriche hermaphroditica

Epilobium adenocaulon

Pyrola secunda

Potentilla egedii

Gentiana glauca

Dodecatheon pulchellum

Saxifraga tricuspidata

Antennaria monocephala

Prenanthes alata

Sedum rosea

Drosera rotundifolia

Potarnogeton pectinatus

Angelica lucida

Triglochin maritimum

Ranunculus cymbalaria

Honckenya peploides

Myriophyllum sibiricum

Moneses uniflora

Sanguisorba stipulata

Stellaria sitchana

Osmorhiza purpurea

Valeriana sitchensis

Lysichiton americanum

Platanthera saccata

Epilobium leptocarpum

Potamogeton filiformis

Sparganium minimum

Pedicularis parviflora

Pyrola minor

Callitriche verna

Gentiana platypetala

Trientalis europaea

Swertia perennis

Tofieldia glutinosa

Erigeron peregrinus

Gentiana douglasiana

Epilobium palustre

Polemonium acutiflorum

Epilobium angustifolium 
threepetal bedstraw

trifoliate goldenthread

tufted loosestrife

twisted stalk

western columbine

yarrow

yellow anemone

yellowdot saxifrage

yellow marsh-marigold

yellow pondlily

yellow rattle

yellow violet

wedgeleaf primrose

western buttercup

western dock

western hemlock-parsley

western twayblade

western water hemlock

white water crowfoot

whorled lousewort

wild iris

woolly hawkweed

woolly lousewort

vetchling

\section{GRAMINOIDS}

Alaska bentgrass

alpine holygrass

alpine rush

arctic bluegrass

arctic bentgrass

arctic rush

arctic sedge

beach rye

bluejoint reedgrass

boreal bog sedge

common spikerush

common woodrush

dwarf alkaligrass

false mannagrass

fewflower sedge

fewseeded bog sedge

longawned sedge
Galium trifidum $\quad 68$

Coptis trifolia $\quad 60$

Lysimachia thyrsiflora $\quad 81$

Streptopus amplexifolius $\quad 113$

Aquilegia formosa 49

Achillea borealis $\quad 45$

Anemone richardsonii $\quad 47$

Saxifraga bronchialis $\quad 106$

Caltha palustris $\quad 54$

Nuphar polysepala $\quad 86$

Rhinanthus minor $\quad 103$

Viola glabella $\quad 119$

Primula cuneifolia 98

Ranunculus occidentalis $\quad 102$

Rumex fenestratus 105

Conioselinum chinense $\quad 59$

Listera caurina 79

Cicuta douglasii $\quad 56$

Ranunculus trichophyllus $\quad 102$

Pedicularis verticillata $\quad 91$

Iris setosa $\quad 76$

Hieracium triste $\quad 74$

Pedicularis kanei $\quad 89$

Lathyrus palustris $\quad 77$

Agrostis alaskana $\quad 124$

Hierochloë alpina $\quad 136$

Juncus alpinus $\quad 137$

Poa lanata $\quad 140$

Agrostis aequivalvis $\quad 124$

Juncus arcticus 138

Carex anthoxanthea $\quad 127$

Elymus arenarius $\quad 133$

Calamagrostis canadensis $\quad 126$

Carex magellanica $\quad 129$

Eleocharis palustris $\quad 133$

Luzula multiflora $\quad 138$

Puccinellia pumila $\quad 142$

Glyceria pauciflora $\quad 136$

Carex pauciflora $\quad 130$

Carex microglochin $\quad 130$

Carex macrochaeta $\quad 128$ 
Lyngbye's sedge

manyflower sedge

meadow barley

mountain hairgrass

mountain timothy

needle spikerush

Nootka alkaligrass

Pacific reedgrass

pendant grass

polargrass

red cottongrass

red fescue

rough fescue

seashore bluegrass

Sitka sedge

smallflowered woodrush

smallawned sedge

spike trisetum

tall cottongrass

tufted bulrush

tufted hairgrass

Wahlenberg's woodrush

water sedge

FERNS AND FERN ALLIES

alpine clubmoss

beech fern

club spikemoss

common horsetail

deer fern

fir clubmoss

fragile fern

groundcedar

lady fern

maiden fern

marsh horsetail

meadow horsetail

northern horsetail

oak fern

running clubmoss

savin groundpine

stiff clubmoss

swamp horsetail
Carex lyngbyaei

Carex pluriflora

Hordeum brachyantherum

Vahlodea atropurpurea

Phleum commutatum

Eleocharis acicularis

Puccinellia nutkaensis

Calamagrostis nutkaënsis

Arctophila fulva

Arctagrostis latifolia

Eriophorum russeolum

Festuca rubra

Festuca altaica

Poa macrantha

Carex sitchensis

Luzula parviflora

Carex microchaeta

Trisetum spicatum

Eriophorum angustifolium

Trichophorum caespitosum

Deschampsia caespitosa

Luzula wahlenbergii

Carex aquatilis

Lycopodium alpinum

Thelypteris phegopteris

Selaginella selaginoides

Equisetum arvense

Blechnum spicant

Lycopodium selago

Cystopteris fragilis

Lycopodium complanatum

Athyrium filix-femina

Thelypteris limbosperma

Equisetum palustre

Equisetum pratense

Equisetum variegatum

Gymnocarpium dryopteris

Lycopodium clavatum

Lycopodium sabinifolium

Lycopodium annotinum

Equisetum fluviatile 
wood fern

woodland horsetail
Dryopteris dilatata

Equisetum sylvaticum
147

150 


\section{Appendix 8. Suggested reading.}

I. Southcentral and Southeast Alaska Plant Community Guides:

Boggs, K. 2000. Classification of community types, successional sequences, and landscapes of the Copper River Delta, Alaska. General Technical Report PNW-469, USDA Forest Service, Pacific Northwest Research Station, Portland, OR. 244 p.

DeMeo, T., J. Martin, and R.A. West. 1992. Forest plant association management guide, Ketchikan Area, Tongass National Forest. Alaska Region publication R10-MB-210, USDA Forest Service, Juneau, AK. 405 p.

DeVelice, R.L., C.J. Hubbard, K. Boggs, S. Boudreau, M. Potkin, T. Boucher, and C. Wertheim. 1999. Plant community types of the Chugach National Forest: southcentral Alaska. Alaska Region Technical Publication R10-TP-76, USDA Forest Service, Chugach National Forest, Anchorage, AK. 375 pp.

Martin, J.R., S.J. Trull, W.W. Brady, R.A. West, and J.M. Downs. 1995. Forest plant association management guide, Chatham Area, Tongass National Forest. Alaska Region Technical Publication R10-TP-57. USDA Forest Service, Juneau, AK.

Pawuk, W.H. and E. Kissinger. 1988. Preliminary forest plant associations of the Stikine Area, Tongass National Forest. Alaska Region Technical Publication R10-TP-72. USDA Forest Service, Juneau, AK. 126 p.

Shephard, M.E. 1995. Plant community ecology and classification of the Yakutat foreland, Alaska. Alaska Region Technical Publication R10-TP-56. USDA Forest Service, Juneau, AK. 214 p. (plus appendices)

II. Plant Books:

Borchers, S.L. and C. Wertheim. 1992. Forest plant identification guide: Chugach National Forest. Alaska Region publication R10-MB-189, USDA Forest Service, Juneau, AK. 124 p.

Hultén, E. 1968. Flora of Alaska and neighboring territories: A manual of the vascular plants. Stanford University Press, Stanford, CA. 1008 p.

LaBounty, K. and J.R. Martin. 1987. Plant identification guide for forested sites of the Tongass National Forest. USDA Forest Service, Tongass National Forest, Sitka, AK. 71 p. 
II. Plant Books, continued:

Pojar, J. and A. MacKinnon. 1994. Plants of the Pacific Northwest coast:

Washington, Oregon, British Columbia \& Alaska. Lone Pine Publishing, Vancouver, British Columbia. 527 p.

Robuck, O. Wayne. 1985. The common plants of the muskegs of southeast Alaska. Pacific Northwest Research Station Miscellaneous Publication. USDA Forest Service, Portland, OR. 131 p.

Robuck, O. Wayne. 1989. Common alpine plants of southeast Alaska. Pacific Northwest Research Station Miscellaneous Publication. USDA Forest Service, Portland, OR. $207 \mathrm{p}$.

Viereck, L.A. and E.L. Little, Jr. 1972. Alaska trees and shrubs. USDA Forest Service, Agriculture Handbook No. 410. Washington, D.C. 265 p.

Vitt, D.H., J.E. Marsh, and R.B. Bovey. 1988. A photographic field guide to the mosses, lichens, and ferns of northwest North America. Lone Pine Publishing, Edmonton, Alberta. $296 \mathrm{p}$.

Welsh, S.L. 1974. Anderson's flora of Alaska and adjacent parts of Canada. Brigham Young University Press, Provo, Utah. 724 p.

III. Scientific Nomeclature and Species Codes:

USDA, NRCS 1999. The PLANTS database (http://plants.usda.gov /plants). National Plant Data Center, Baton Rouge, LA 70874-4490 USA. 

NATIONAL AGRICULTURAL LIBRARY

1022493207 
* national agricultural librapy

national agr

1022493207 